\title{
Comprehensive Invited ReView
}

\section{Multiple Sclerosis: Molecular Mechanisms and Therapeutic Opportunities}

\author{
Djordje Miljković ${ }^{\text {and Ivan Spasojević }}{ }^{2}$
}

\begin{abstract}
The pathophysiology of multiple sclerosis (MS) involves several components: redox, inflammatory/autoimmune, vascular, and neurodegenerative. All of them are supported by the intertwined lines of evidence, and none of them should be written off. However, the exact mechanisms of MS initiation, its development, and progression are still elusive, despite the impressive pace by which the data on MS are accumulating. In this review, we will try to integrate the current facts and concepts, focusing on the role of redox changes and various reactive species in MS. Knowing the schedule of initial changes in pathogenic factors and the key turning points, as well as understanding the redox processes involved in MS pathogenesis is the way to enable MS prevention, early treatment, and the development of therapies that target specific pathophysiological components of the heterogeneous mechanisms of MS, which could alleviate the symptoms and hopefully stop MS. Pertinent to this, we will outline (i) redox processes involved in MS initiation; (ii) the role of reactive species in inflammation; (iii) prooxidative changes responsible for neurodegeneration; and (iv) the potential of antioxidative therapy. Antioxid. Redox Signal. 19, 2286-2334.
\end{abstract}

$\begin{array}{ll}\text { I. Introduction } & 2287\end{array}$

$\begin{array}{ll}\text { A. Basic facts } & 2287\end{array}$

B. Autoreactive T cells $\quad 2289$

C. The immune privilege of the central nervous system $\quad 2290$

D. Animal models of MS

1. Experimental autoimmune encephalomyelitis 2293

2. Theiler's murine encephalitis virus-induced demyelinating disease 2293

3. Cuprizone 22293

II. Redox Processes in the Initiation of MS 2293

A. The baseline characteristics of initial lesion 2293

B. Circulatory abnormalities in pre-MS brain $\quad 2294$

C. Blood-brain nitric oxide crosstalk 2295

D. $\mathrm{NO}$ and $\mathrm{H}_{2} \mathrm{O}_{2}$ from endothelium activate the redox cycle in astrocytes 2297

E. The decrease of NADH/NAD ${ }^{+}$ratio 2297

F. Astrocytes and macrophages: centers of oxidative/nitrosative stress in early lesions 2298

G. Oligodendrocytes death 2298

H. Demyelination $\quad 2299$

I. BBB breakdown $\quad 2299$

J. The development of autoimmunity 2301

1. Antigens 2301

2. The activation/reactivation of T cells 2302

K. Redox regulation of T-cell migration and infiltration $\quad 2303$

L. The outside-in initiation of MS $\quad 2304$

Reviewing Editors: Vittorio Calabrese, Ed Croze, Gianna Ferretti, Betty Miller, Ken Smith, Jose M. Soria and Maarten Witte

${ }^{1}$ Department of Immunology, Institute for Biological Research "Siniša Stanković," University of Belgrade, Belgrade, Serbia.

${ }^{2}$ Life Sciences Department, Institute for Multidisciplinary Research, University of Belgrade, Belgrade, Serbia. 
III. Redox Processes in Inflammation 2306

A. Redox processes in macrophages 2306

B. Redox processes in T cells 2307

C. Cytokines and the intracellular oxidative stress 2309

D. The contribution of oxidative stress to relapses and progression 2309

E. Vitagene network, heat shock proteins, and inflammation 2310

IV. Neurodegeneration in MS 2311

A. "Slow burning" of demyelinated axons 2311

B. Neurodegeneration in acute lesions 2311

$\begin{array}{ll}\text { 1. White matter } & 2311\end{array}$

2. Cortex 2312

C. Inappropriately chelated iron 2313

V. Redox Therapy in MS 2314

A. General strategy 2314

B. Fumarates

C. Cannabinoids

D. The inhibition of NF- $\kappa \mathrm{B}$ and iNOS 2316

E. Other agents 2317

1. Iron chelators 2317

2. N-Acetylcysteine 2317

3. Ketogenic diet 2317

$\begin{array}{ll}\text { 4. Vitagene network activators } & 2317\end{array}$

VI. Concluding Remarks 2317

\section{Introduction}

\section{A. Basic facts}

$\mathbf{M}$ ULTIPLE SCLEROSIS (MS) is an inflammatory demyelinating disease of the central nervous system (CNS). It is a leading cause of neurological disabilities in young adults and affects approximately $0.24 \%$ of the population in the United States and Canada, and approximately $0.19 \%$ in some European countries. Clinical expressions of MS vary among the patients depending on the location of affected nerve fibers, but the most common symptoms are sensory loss (paresthesias), motor spinal cord symptoms (numbness or weakness in one or more limbs), autonomic spinal cord symptoms (bladder, bowel, and sexual dysfunction), cerebellar symptoms (dysarthria, ataxia, and tremor), optic neuritis (partial or complete loss of central vision), other eye symptoms (blurred or doubled vision), trigeminal neuralgia, facial myokymia, heat intolerance, fatigue, dizziness, lack of sleep, pain, subjective cognitive difficulties, and depression.

MS takes different clinical courses in patients $(75,192)$. The most common is the relapsing-remitting course (progressiverelapsing MS [RRMS]) in which flare-ups of neurological disabilities occur from time to time, followed up by a complete or partial recovery. In some of these patients, neurological disabilities accumulate without proper recovery, that is, MS takes a secondary progressive course (SPMS). In other patients, the progressive course is taken from the beginning of clinically manifested MS, and this course is called primary progressive MS (PPMS). There is also a progressive form of MS that is combined with occasional relapses, that is, PRMS. There are also patients who have rare attacks of the disease and always show complete recovery, and such a course of MS is called benign. Finally, there are cases of clinically isolated syndrome (CIS), that is, one episode of neurological deficits which does not always progress into definite MS. The disease is also heterogeneous according to histopathological findings in MS patients. Based on histopathology and pathogenic mechanisms, four subtypes of MS have been identified (258). We shall address this later in relation to redox changes.

Epidemiological data indicate that both genetic and environmental factors are important for the etiology of MS (184). It is known that some major histocompatibility complex (MHC) haplotypes, as well as some alleles of cytokines and their receptors, increase the risk for MS (174). Environmental risk factors include infections, inadequate exposure to sunlight, and smoking $(23,184)$. An interaction between environmental and genetic factors in the susceptibility to MS is presumed to be very complex. Still, some recent findings revealed possible scenarios for collaboration between specific factors in MS initiation. For instance, MS risk modulators, including genetic variants in interleukin-7 receptor- $\alpha\left(I L 7 R A^{*} \mathrm{C}\right)$, interleukin-2 receptor- $\alpha$ (IL2RA*T), MGAT1 (IVAVT-T) and CTLA-4 (Thr17Ala), and environmental factors affecting vitamin D3 levels, converge in order to alter branching of Asn (N)-linked glycans (323). Importantly, branching reduction results in T-cell hyperactivity and promotes spontaneous inflammatory demyelination and neurodegeneration in the MS animal model (176). N-glycan branching is positively regulated by the Golgi enzymes Mgat1 and/or Mgat5. Down-regulation of Mgat1 by $I L 7 R A^{*} C$ and IL2RA*T is opposed by MGAT1 (IVAVT - T) and vitamin D3, which optimizes branching and mitigates the risk of MS when combined with enhanced N-glycosylation of CTLA-4. Therefore, various genetic and environmental factors regulate a final common pathway-Nglycosylation-which is highly relevant for MS pathogenesis. In addition, it has been shown that vitamin $\mathrm{D}$ directly stimulates the expression of $H L A-D R B 1^{*} 15$, MHC class II allele, which is a major MS genetic risk factor $(174,373)$. It has been suggested that a low vitamin $\mathrm{D}$ level in early childhood leads to a decrease in the expression of $H L A-D R B 1^{*} 15$ in the thymus and, therefore, to inadequate presentation of self-antigens and inefficient central tolerance (for more details on these 
processes, see the next section) (186). As a consequence, more autoreactive $\mathrm{T}$ cells could emerge in the immune system of people who had low vitamin D in their early childhood. To make the story even more complex, low vitamin D levels may be a consequence of genetic malfunction, for example, nonfunctional vitamin D converting enzyme CYP27B1, which converts 25-hydroxyvitamin D to 1,25-dihydroxyvitamin D (174), or a consequence of an environmental influence, that is, low exposure to sunlight or an inadequate diet. The latter might explain why a higher frequency of $H L A-D R B 1^{*} 15$ is detected in MS patients in Scandinavian countries compared with those in Mediterranean countries, or those in some other non-European countries (391).

MS investigation is very vivid, as many elements of its complex pathogenesis are still unknown, and (consequently) there are no medications for curing the patients with the disease. Various therapies, which are designed to affect immune response directed against the $\mathrm{CNS}$, are being applied, but they only slow down and/or reduce severity of the clinical course of MS. This disappointing fact emphasizes the importance of other components in MS pathology. Numerous studies, performed mainly in the last decade, suggest that redox processes have a critical role in all stages of MS, particularly in the firstthe initiation, and the last- the neurodegeneration. However, before we address the various involvements of redox alterations in MS initiation and progression, we should introduce the readers to the major cell types of the CNS and the immune system involved in the pathogenesis of the disease, as well as to some of their properties that are relevant for MS pathogenesis (details are given in Fig. 1).

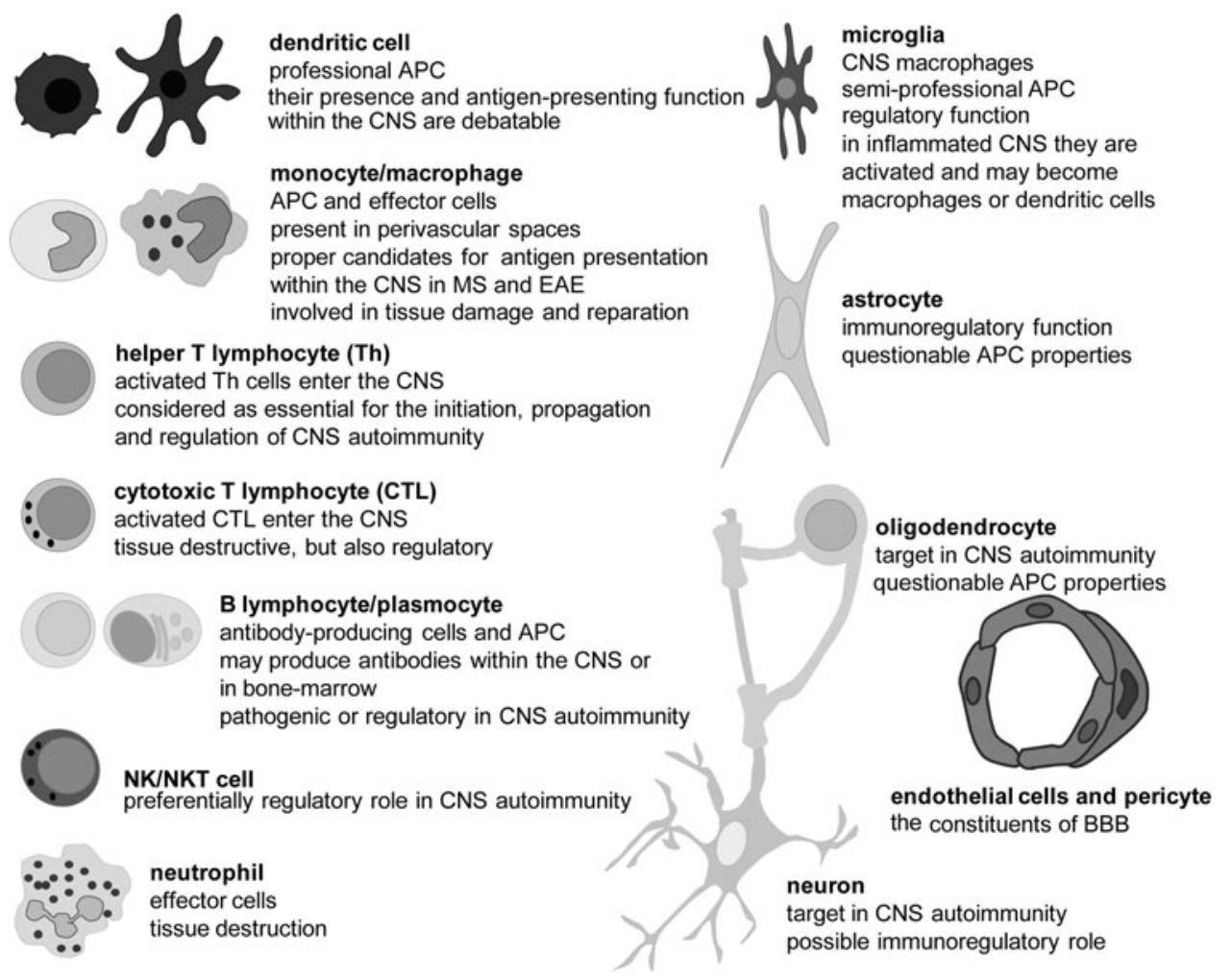

FIG. 1. The main cells of the immune system (on the left) and the CNS (on the right) involved in MS pathogenesis. DC are professional APC that play a central role in the initiation of the adaptive immune response (98). Macrophages are phagocytic cells and powerful effectors that are capable not only of killing microbes and infected cells, but also of damaging self-tissue. T cells and B cells are effectors in adaptive immunity. Th produces soluble mediators, which act through receptors in other immune and non-immune cells. CTL kill other cells. B cells produce antibodies (immunoglobulins). In response to antigens, B cells mature and become plasma cells. Neutrophils are innate immunity phagocytes and powerful effector cells that are capable of eliminating pathogens. Neutrophils are absent from the healthy CNS, unlike T and B cells that are present in limited numbers (375). However, all these immune cells readily infiltrate the CNS in various diseases, including MS and its animal models. Microglial cells are resident macrophages of the CNS. Although seemingly in a quiescent state, microglia cells carry out active CNS tissue scanning (187). After activation, microglial cells may become classical phagocytic cells, which eliminate cell debris and infected cells (139). Astrocytes are the most numerous cells of the human brain. They provide an optimal physical and metabolic environment for neuronal activities. Astrocytes are crucial for neuronal redox homeostasis, potassium balance, BBB regulation, myelination, neurotransmitter uptake, and synapse formation and function. Neurons transmit information by electrical signaling. In order to efficiently conduct signals, their long processes (axons, neurites) should be insulated with myelin. Oligodendrocytes are cells that provide support and insulation for axons by forming the myelin sheath. A single ODC can extend its processes to approximately 50 axons. The sheaths may be thicker than $1 \mu \mathrm{m}$, and they are composed of lipids $(80 \%)$ and proteins $(20 \%)$. Endothelial cells and pericytes are constituents of small BVs within the CNS and have important functions in CNS-immunity relation, especially regarding BBB. APC, antigen-presenting cells; BBB, blood-brain barrier; CTL, cytotoxic T cells; DC, dendritic cells; ODC, oligodendrocytes; BV, blood vessel. 


\section{B. Autoreactive T cells}

T cells, which are essential for MS pathogenesis, are autoreactive, that is, they react with self-antigens of the CNS. Such $\mathrm{T}$ cells initiate and propagate an autoimmune response against the CNS and orchestrate cellular and humoral effectors, which attack myelin, oligodendrocytes (ODC), and neurons. They are also one of the important effectors involved in the CNS damaging. The essential signal for T-cell activation comes from the recognition of antigens presented by MHC molecules on the surface of antigen-presenting cells (APC). In order to efficiently present an antigen to a naïve T cell, APC have to express a complex of antigens and MHC molecules, as well as co-stimulatory molecules, and to produce adequate cytokines (95). Dendritic cells (DC) are APC that are capable of activating naïve T cells; hence, they play a central role in the initiation of the adaptive (auto)immune response (98). T cells contact antigen/MHC complexes with their T-cell receptors (TCR) and co-receptor molecules (CD4 or CD8), and in addition, bind co-stimulatory molecules of APC (Fig. 2A). If a $\mathrm{T}$ cell expresses CD4, it recognizes MHC class II molecules, and if it expresses CD8, it recognizes MHC class I molecules. Most of the $\mathrm{T}$ cells express exclusively CD4 or CD8, and are, therefore, designated as CD ${ }^{+} \mathrm{T}$ cells and $\mathrm{CD} 8^{+} \mathrm{T}$ cells, with the former being helper $\mathrm{T}$ cells (Th) and the latter being cytotoxic T cells (CTL). In addition, among both populations, a certain proportion belongs to regulatory T cells (Treg). Th cells produce soluble mediators that perform effector and regulatory functions in immunity, while CTL use perforin and granzymes to kill infected, transformed, or damaged cells. Th cells are particularly important for the pathogenesis of MS, because they play a crucial role in the maturation and complete activation of other immune cells. Without the support of Th cells, B cells do not mature into long-lasting memory cells or plasma cells (Fig. 2B). CTL are also not fully activated without the support from Th cells (Fig. 2A). Similarly, macrophages and neutrophils will not be properly engaged in adaptive immunity without the contribution of Th cells. There are several populations of effector Th cells, including autoreactive Th1 and Th17, which appear to be the major pathogenic populations in MS (362). Importantly, in order to perform their effector functions, $T$ cells have to be reactivated once they enter the CNS. It is generally accepted that there are no DC in the healthy CNS (375). Still, other APC are capable of reactivating $\mathrm{T}$ cells in the CNS. For instance, perivascular, meningeal, and choroid plexus macrophages are present in healthy CNS (375). Besides macrophages, CNS contains other semi-professional APC (microglia, B cells, and endothelial cells) and non-professional APC (astrocytes), which can reactivate autoreactive $\mathrm{T}$ cells $(12,38,109,440)$.

TCR are being generated stochastically by somatic recombinations and, therefore, T cells expressing TCR that recognize self-antigens are produced in the immune system. Still, in the process of maturation, such $\mathrm{T}$ cells are eliminated or
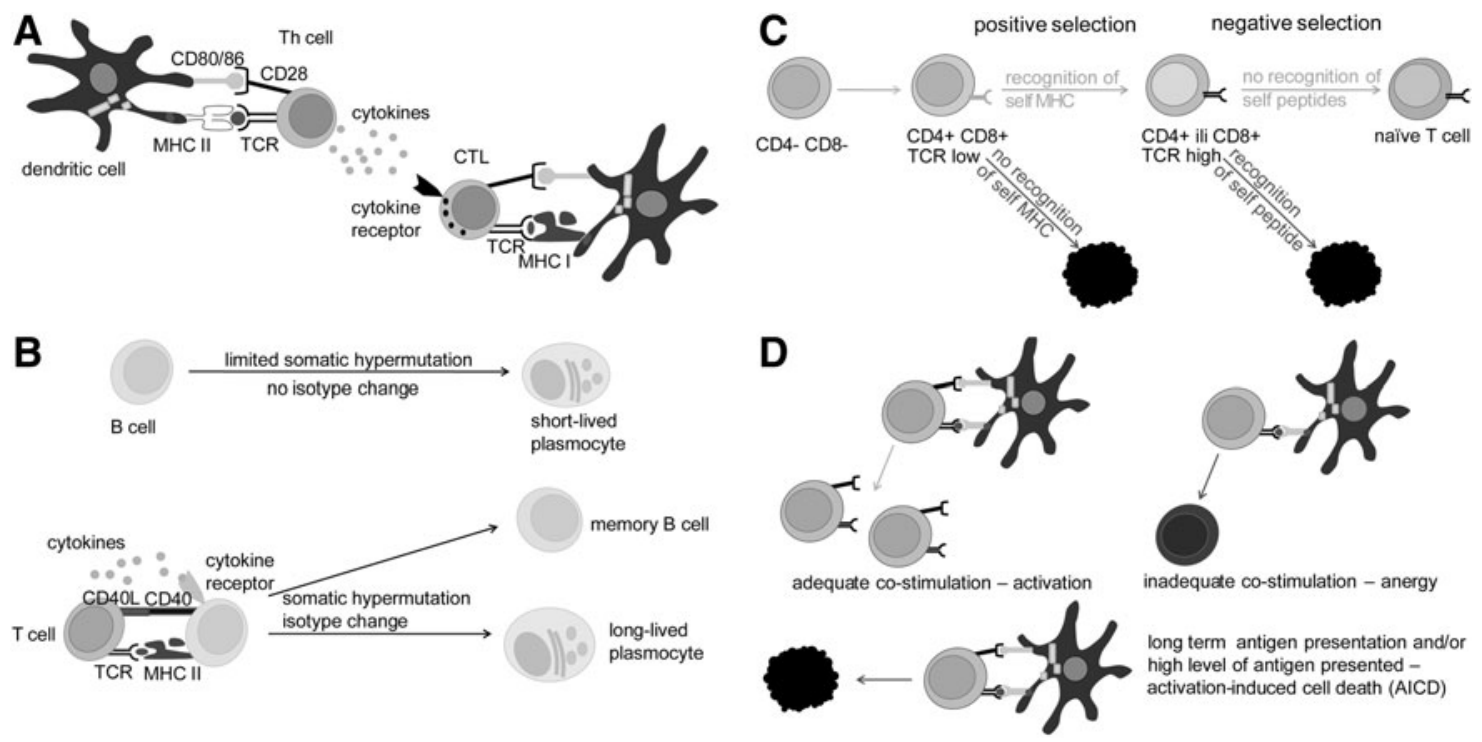

FIG. 2. The activation of CTL and B cells and the maturation of T cells. (A) MHC class II and TCR/CD4 make the central recognition pair between APC and Th cells. MHC class I and TCR/CD8 make the central recognition pair between APC and CTL. CD80/86 and CD28 make co-stimulatory pairs in both cases. Cytokines, produced by Th cells, are needed for complete activation of CTL. (B) Only B cells activated with the help of Th cells differentiate into memory B cells and highly efficient plasmocytes. Their immunoglobulins (either soluble antibodies or B-cell receptors) are adjusted for antigen recognition and effector functions through processes of somatic hypermutation and isotype switch, respectively. (C) Central selection takes place in the thymus. There, T cells commit to CD4 or CD8 lineage. In addition, $\mathrm{T}$ cells that interact with MHC-peptide complexes too weakly or too strongly are eliminated in positive and negative selection. (D) Peripheral selection takes place outside primary immune organs. T cells that recognize foreign peptides are adequately stimulated, as they receive proper costimulation by APC. T cells that recognize self-peptides are inadequately stimulated and eliminated. For this, there are at least two options. First, T cells may contact APC that does not provide adequate co-stimulation and as a consequence, instead of activation, anergy is induced in these T cells. Alternatively, $\mathrm{T}$ cells that are activated for too long as a consequence of longterm expression of self-antigens on APC will undergo AICD. AICD, activation-induced cell death; MHC, major histocompatibility complex; TCR, T cell receptor. 
inactivated by mechanisms of central and peripheral tolerance. Central tolerance is based on the interaction between TCR and MHC. It takes place in the thymus and involves two steps-positive and negative selection (175). Every subject has a highly specific repertoire of MHC molecules that are expressed in the cells. This fact is the base for positive selection, as many of the T cells produced in any subject will not be able to interact appropriately with the MHC molecules that are expressed in the thymus and will, therefore, be eliminated (Fig. 2C). Nevertheless, some of these cells that are capable of recognizing antigens in the complex with MHC molecules present in the thymus will interact very strongly with the MHC-antigen complex. Such T cells will be eliminated in the process of negative selection (Fig. 2C). This is very important for the prevention of T-cell autoreactivity, as antigens expressed in the thymus are self-antigens, and, therefore, the eliminated $\mathrm{T}$ cells would have been potential candidates for autoreactivity. Ideally, after positive and negative selection, naïve $\mathrm{T}$ cells generated in the thymus should be able to interact with MHC molecules that express foreign, but not selfantigens. However, negative selection is not perfect. Not all of the antigens are expressed in the thymus, and, therefore, some of the T cells with a potential to recognize self-antigens will survive the selection. Typical antigens that are not expressed in the thymus are those whose expression is limited to embryonic development and those which are specific for certain tissues (so-called tissue specific antigens [TSA]). Given that some of the CNS antigens are TSA, T cells that are potentially reactive to CNS antigens are present in our immune system. It can be speculated that CNS antigens are TSA due to the immune privilege of CNS, which developed in order to provide the "control center" with high protection (see the next section), but leave the immune system non-introduced to certain CNS antigens.

At the periphery of the immune system (every lymphoid organ and tissue outside the bone marrow and thymus), $\mathrm{T}$ cells that recognize antigens in an inadequate manner could be eliminated or become anergic (Fig. 2D). A naïve T cell can be activated only if it receives simultaneous stimulation through TCR, co-receptors, and cytokines (95). If the co-stimulation is absent, even though primary TCR-antigen$\mathrm{MHC}$ recognition is perfect, a T cell will become irresponsive anergic. In addition, if the recognition lasts too long and/or is too intense, a T cell will be eliminated in the process of activation-induced cell death (AICD). Typically, self-antigens are presented in the complex with MHC molecules for a long time and in many copies, which makes them perfect candidates for AICD induction. It is also important to note that Treg are actively involved in peripheral tolerance, restraining autoreactivity (96). Findings that suggest disturbances in their development and function in MS patients strongly imply the importance of peripheral tolerance for prevention of the disease (450). As already stated, Th cells are crucial for complete activation of macrophages, neutrophils, CTL, and B cells (Fig. 2A, B). Therefore, if self-tolerance mechanisms fail at the level of Th cells, the road is paved for other populations of immune cells to become autoreactive.

Due to the imperfections of tolerance mechanisms, the activation of self-reactive $\mathrm{T}$ cells with self-antigens, specific modifications of self-antigens, or microbes is possible $(22,82)$. Some of the major molecular targets in the immune response in MS and its models are identified. These are myelin proteins, including myelin oligodendrocyte glycoprotein (MOG), myelin basic protein (MBP), and proteolipid protein (PLP); lipids, such as sulfatide, sphingomyelin, and oxidized lipids; and glycans [e.g., $\operatorname{Glc}(\alpha 1,4) \operatorname{Glc}(\alpha)](114)$. Axo-glial proteins, such as neurofascin and contactin-2, are also important molecular targets in MS (114). Modification of self-molecule antigenicity as a consequence of redox changes in the CNS might be essential for the initiation of autoimmunity in MS pathogenesis, as discussed in detail in section II.J.1. Finally, microbes might be involved in the primal activation of encephalitogenic $\mathrm{T}$ cells. There are at least three mechanisms of activation of self-reactive $\mathrm{T}$ cells involving microbes: molecular mimicry, superantigens, and bystander activation. These are described in detail in Figure 3.

\section{The immune privilege of the central nervous system}

Although autoreactive $\mathrm{T}$ cells exist in the immune repertoire, they are no guarantee for the induction of immune response against the CNS components. It was shown, about 30 years earlier, that peripheral blood of healthy individuals contains $\mathrm{T}$ cells that are specific for myelin antigens (e.g., $\mathrm{MBP}$ ) (63). Moreover, the frequency of myelin-reactive T cells in the blood of MS patients is not increased compared with that of healthy controls (44). Bearing in mind the incidence of MS, it is obvious that the activation of the autoreactive cells is more of an exemption than a rule. The base for the impediment of CNS-directed autoimmune reactivity lies in the fact that CNS is not an ordinary system in relation to the immune system, because it is immune privileged (Fig. 4).

The immune privilege of the CNS is based on the bloodbrain barrier (BBB) and blood-cerebrospinal fluid (CSF) barrier (BCSFB), which actively regulate the transport of molecules and cells between systemic circulation and CNS parenchyma (Fig. 4A). BBB is composed of endothelial cells that make strong interconnections-tight junctions (TJ) (132). Astrocytes and pericytes are essential for the formation and function of BBB (465). Postcapillary venules are blood vessels that are relevant to immune cell infiltration into the CNS. There, the immune cells have to cross the endothelial cell layer and endothelial basal lamina and to enter perivascular space (Virchow-Robin space [VRS]) (40). This space is limited by endothelial basal lamina and glial basal lamina. Astrocytic end-feet lie beyond glial basal lamina. Both these make glia limitans. The entrance of immune cells into the VRS is not considered classical infiltration. Instead, immune cells have to pass the glia limitans and enter the CNS parenchyma in order to infiltrate the CNS (40). Although the complex structure impedes immune cell entry into the CNS, macrophages and activated T cells of any specificity are capable of passing BBB and BCSFB and entering the CNS $(199,459)$. In addition, there are regions of the CNS where BBB is incomplete, such as circumventricular organs (147). These have a specific immune status and have been associated with neuroinflammation (468). Still, in general, the transport of antibodies and cells from immune organs and tissues into the CNS is impaired due to the BBB and BCSFB, and may be promoted in the case of BBB dysfunction and breakage.

Another specificity of the CNS is underdeveloped lymphatic drainage. There are no classical lymphatic vessels that drain brain and spinal cord. Instead, cerebral extracellular 


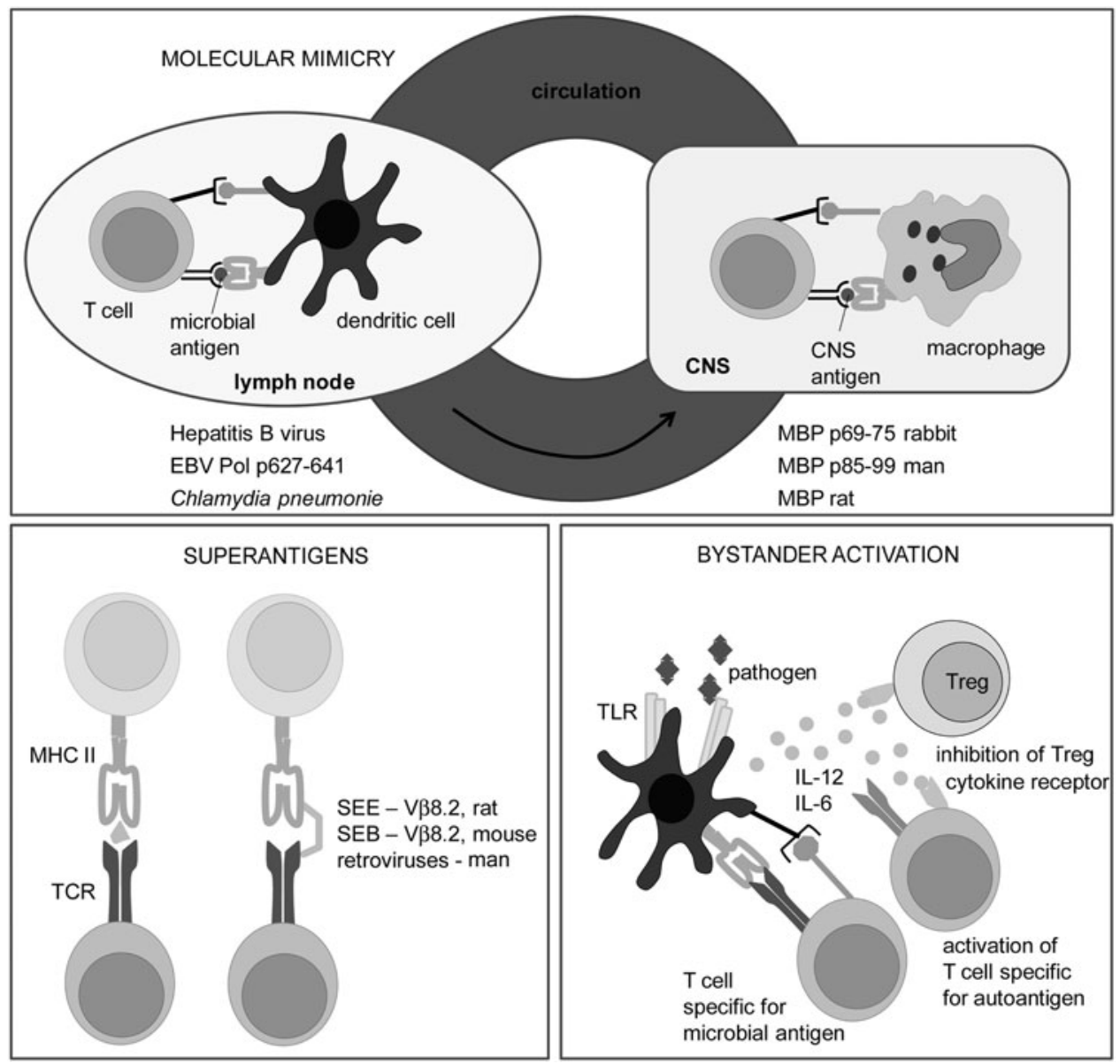

FIG. 3. The activation of autoreactive $\mathbf{T}$ cells by microbes. The examples of possible contribution of microbes to the activation of self-reactive T cells are presented. Molecular mimicry is a process in which T cells are activated with microbial antigen, but since its TCR also recognizes a certain autoantigen, the same T cell will be reactivated with this autoantigen. It is important to understand that when we say that TCR has a single specificity, it does not imply that it recognizes only one peptide. It is actually recognizing the physical, rather than chemical, structure of a peptide, lipid, or saccharide (88). Therefore, if there are two different peptides with a highly similar shape, TCR will not be able to tell them apart. Some examples of microbial and self-antigens relevant for MS that are recognized by the same TCR are given (EBV Pol-EpsteinBarr virus DNA polymerase). Superantigens are microbial products that activate many clones of T cells. A superantigen is not recognized by T cells in a classical way (left side of the panel). Instead, it bypasses MHC and TCR that has certain $\alpha$ or $\beta$ chain (right side of the panel). Thus, all T cells in an individual that express TCR with such an $\alpha$ or $\beta$ chain are potentially activated. As a consequence of such a broad, oligoclonal, or polyclonal activation, some autoreactive T cells may be activated, as well. Some superantigens that are relevant for MS, as well as $\beta$ chains of TCR that they bind to are listed (SEE, SEB, V $\beta$ ). Bystander activation of T cells occurs in an infection when Tregs are inhibited and APC are stimulated by microbes. The pathogens are usually recognized by APC through pattern recognition receptors, such as Toll-like receptors (TLR). As a consequence, APC are stimulated not only to express co-stimulatory molecules, but also to generate cytokines that inactivate Treg. Therefore, if these APC present autoantigens, autoreactive T cells may be efficiently stimulated, as they will receive adequate co-stimulation and as Treg will not suppress their activation. Treg, regulatory T cells; SEE, Staphylococcal enterotoxin E; SEB, Staphylococcal enterotoxin $\mathrm{B} ; \mathrm{V} \beta$, variable region of $\beta$ chain of TCR.

fluids drain into the blood across the arachnoid villi and into the lymph along certain cranial nerves and spinal nerve root ganglia. The interstitial fluid of the CNS drains via perivascular channels into the CSF, which is directly reabsorbed into blood via arachnoid villi (375). CSF drains into local afferent lymphatics along cranial and spinal nerves, for example, along olfactory rootlets to the nasal mucosa and then into the deep cervical lymph nodes. While the transport of soluble matters from the CNS into the draining lymph nodes is adequate, cellular transport is heavily impaired (147) (Fig. 4B). There is a special interest in the ability of APC to leave the $\mathrm{CNS}$ and nest into lymphatic organs. Although there is no direct connection between CNS parenchyma and lymph nodes, APC can travel from the CNS to the periphery via CSF and blood (201). This is important in relation to initial antigen presentation in MS (83). It is conceivable that antigen presentation to naïve T cells occurs in the lymph nodes, and, thus, DC have either to collect the antigen within the CNS and then to travel to the draining lymphoid tissue, or to trap a soluble antigen which is transported through lymph vessels. The existence of both soluble and cellular drainage from the CNS to lymph organs is essential for the initial steps of inflammation seeded within the CNS. Even if the initial antigen derives from a microorganism or some other external source and APC 

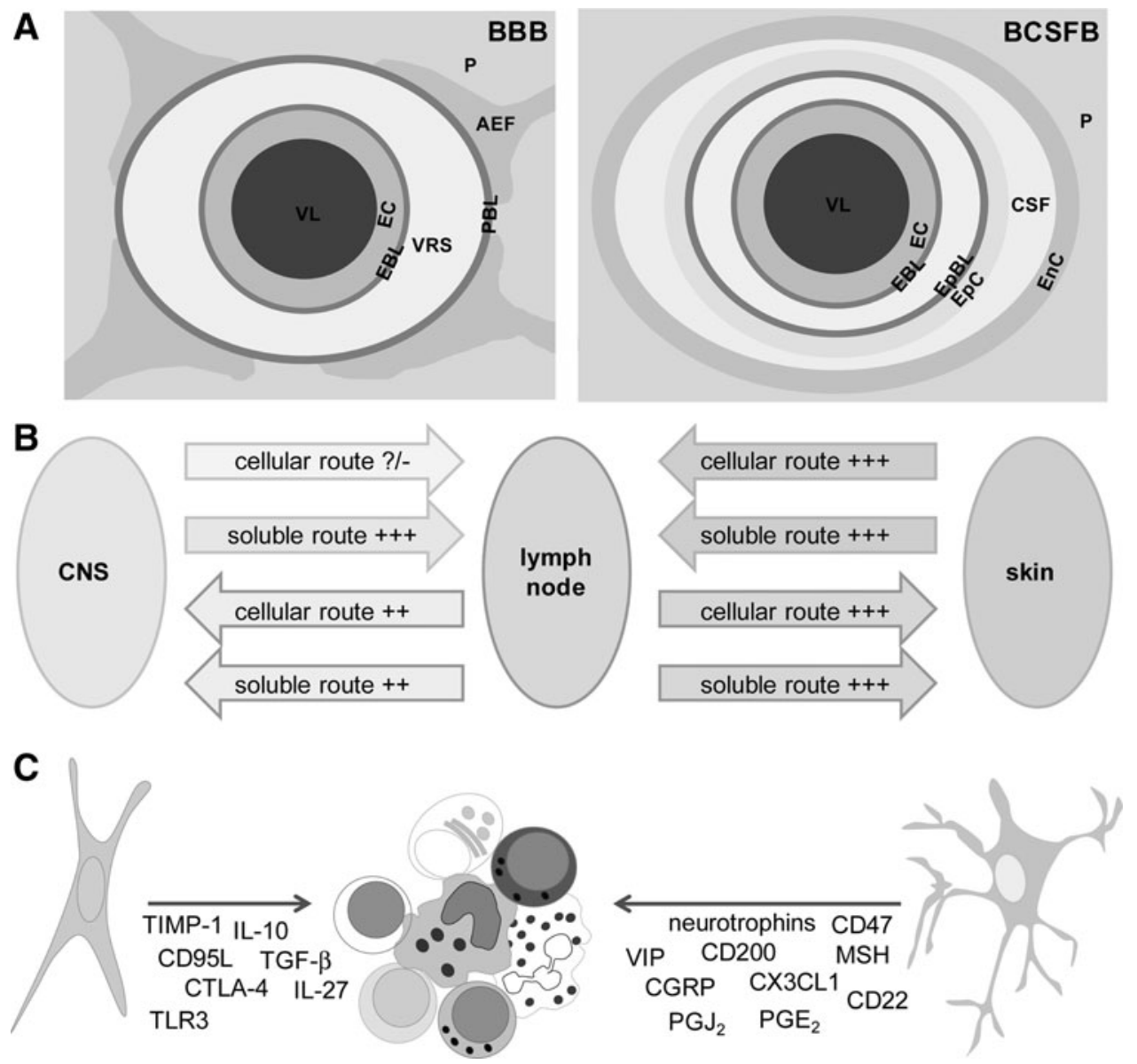

FIG. 4. The elements of the CNS immune privilege. (A) VL, vessel lumen; EC, endothelial cells; EpC, epithelial cells of choroid plexus; EnC, ependymal cells; EBL, endothelial basal lamina; EpBL, epithelial basal lamina; VRS, Virchow-Robin space; PBL, parenchymal basal lamina; AEF, astrocytic end-feet; CSF, cerebrospinal fluid; P, parenchyma. TJ are essential elements of these barriers, and they are present among EC in BBB and among EpC in BCSFB. The EC of BCSFB form fenestrated walls, and they are not particularly important for the barrier function. In order to enter CNS parenchyma, immune cells have to pass EC, EBL and PBL of BBB or EC, EBL, EpBL, EpC, and EnC of BCSFB. (B) Afferent and efferent connections of a lymph node and the skin (as an example of a classical organ) and of a lymph node and the CNS (as an immunoprivileged system). The number of "+" indicates efficacy of the route; "?/-"indicates that it is not certain if the route is operative. (C) Immunosuppressive molecules produced by astrocytes and neurons. BCSFB, blood-CSF barrier.

collect it at the periphery, CNS drainage will still be important for the perpetuation of inflammation and for epitope spreading.

A very important characteristic of the CNS, which contributes to the immune privilege, is the limited presentation of antigens within the CNS. Namely, so far, the presence of DC (the professional APC) in the healthy CNS parenchyma has not been validly documented (375). However, it is known that microglia and macrophages may differentiate into DC-like cells $(368,427)$. Activated microglial cells share numerous properties with DC, including constitutive expression of MHC II in situ and the expression of MHC I on activation (11). Microglia appears to be essential for the activation of $C D 8^{+} \mathrm{T}$ cells. For example, it was shown that transgenic mice overexpressing co-stimulatory molecules on microglial cells develop spontaneous CNS demyelinating disease that is mediated by autoreactive CD8 ${ }^{+} \mathrm{T}$ cells (59). Apart from microglial cells that are the only parenchymal macrophage population, several populations of monocyte/macrophage lineage are situated at the vicinity. These cells are capable of taking samples from the parenchyma as a consequence of their position in the CSF flow pathways. Macrophages are potent APC, as they express MHC class II, co-stimulatory, and adhesion molecules. Furthermore, they are capable of initiating antigen-specific proliferative response in resting $\mathrm{CD}^{+}$ $\mathrm{T}$ cells (375). Finally, it has been reported that $\mathrm{CD}^{+} 4^{+}$monocytes migrate across the inflamed human BBB and that, under the influence of BBB-secreted transforming growth factor- $\beta$ and granulocyte-macrophage colony-stimulating factor, they differentiate into $\mathrm{CD} 3^{+} \mathrm{CD} 209^{+}$DC (216). Importantly, $\mathrm{CD}_{209^{+}}$DC promote proliferation and expansion of Th1 and Th17 lymphocytes, and they are detected in close association with lymphocytes within active MS lesions (397). Monocytes are also present in the CSF, where they constitute around 5\% of the cellular content (375).

Finally, active immunosuppresion performed by CNS resident cells, predominantly astrocytes and neurons, is an important factor of the immune privilege (39) (Fig. 4C). For instance, astrocytes provoke apoptosis in activated T cells in Fas-FasL dependent and independent manner, down-regulate 
proinflammatory cytokine generation in these cells, and even induce Treg (318). Neurons may also down-regulate microglia/macrophage activity (329).

\section{Animal models of $M S$}

Our current knowledge of MS etiopathogenesis is largely based on the studies of its animal models. In this section, we will present frequently used MS models and critically address their place in MS studies.

1. Experimental autoimmune encephalomyelitis. Experimental autoimmune encephalomyelitis (EAE) is the most widely used model of MS. It is an autoimmune disease of the CNS that is actively induced in susceptible animals, including rodents and primates, by immunization with CNS-specific antigens, peptides derived from these antigens, or CNS tissue homogenates. The disease can also be provoked by transfer of encephalitogenic $\mathrm{CD} 4^{+} \mathrm{T}$ cells, obtained from draining lymph nodes of animals immunized for active EAE induction, into the syngeneic animals (transfer or passive EAE). Depending on the species and strain used in experiments, specific requirements (e.g., adjuvants) for effective immunization are imposed, and divergent clinical courses and severity are induced in animals (314). Since it shares many clinical, histological, immunological, and genetic features with MS, EAE has been used in order to gain important insights into MS pathogenesis and to validate new targets for MS therapy (168, 251). However, the credibility of EAE as an MS model has been increasingly criticized [reviewed in Handel et al. (185)]. In short, criticism can be summarized as follows: (i) mice and rats are used in most of the studies of EAE, and these are phylogenetically distant from humans; (ii) the acute monophasic course is characteristic for many EAE variants; (iii) CNS lesions in most of the EAE models are limited to the spinal cord; (iv) demyelination rarely occurs in EAE; and (v) in most EAE variants, $\mathrm{CD} 4^{+}$cells are the major culprits, while some other immune cells might have pathogenic predominance in MS. Taking into account these and other discrepancies between EAE and MS, we have to be careful when translating data from EAE to MS. This is especially important in attempts to test potential MS therapeutics in EAE. Many agents that proved to be beneficial in EAE are inefficient or even detrimental in MS (36). Still, there are some MS therapeutics, including copaxon and natalizumab, the development of which has been based on EAE studies (460). Importantly, numerous molecular and cellular mechanisms of MS pathogenesis have been discovered in EAE research (36, 91, 251, 460). New EAE variants are also emerging, and they are exploring aspects of MS that were previously unaddressed in animal models, for example, the role of $\mathrm{CD} 8^{+} \mathrm{T}$ cells in neuroinflammation $(14,225)$.

2. Theiler's murine encephalitis virus-induced demyelinating disease. Theiler's murine encephalitis virus-induced demyelinating disease (TMEV-IDD) has been intensively used as a model for human demyelinating diseases, including MS $(119,300)$. TMEV is a positive-stranded RNA virus from the family Picornavirade (genus Cardiovirus). The TO subgroup of TMEV induces acute encephalitis between days 5 and 10 postinjection. TMEV-IDD is of particular interest, because it presents a parallel to a possible scenario in humans, where the initial trigger for CNS inflammation and demyelination is a virus. Importantly, clinical signs of TMEV-IDD are similar to those observed in patients with progressive MS, and they include spasticity, incontinence, weakness of the extremities, and, eventually, paralysis (273). As a parallel to the oligoclonal bands found in the CSF of MS patients, intrathecal antibody production has been observed in this model (351). The general criticism for this model is that the non-human pathogen has been used in a model for a human disease. Interestingly, it has recently been reported that a specific type of encephalomyelitis in humans, Vilyuisk encephalitis, might be caused by a human-TMEV recombinant virus (272). It has been shown that TMEV-IDD is especially important for studies of epitope spreading and molecular mimicry (349). Additional viruses, such as murine hepatitis virus, canine distemper virus, coronaviruses, and some retroviruses, are also being used for induction of MS-like demyelinating disease in experimental animals $(257,295,352,441)$.

3. Cuprizone. Finally, demyelination that is induced in mice with a copper chelator cuprizone is an important tool for MS studies $(241,425)$. In this model, ingestion of cuprizone by mice leads to early ODC death, the activation of microglia/ macrophages, and subsequent reversible demyelination (93, 197). This model is particularly useful for studying demyelination and remyelination, and their relation to axonal loss (241). It is highly relevant for the evolution of type III and type IV MS lesions, where changes in ODC appear to be the primary events in disease pathogenesis. The model is also important for studies of demyelination in specific CNS regions, such as hippocampus, which are affected in MS patients but not in other animal models (345). Besides cuprizone, other toxins, such as ethidium bromide and lysolecithin, are being used for the induction of demyelination in experimental animals (441).

\section{Redox Processes in the Initiation of MS}

\section{A. The baseline characteristics of initial lesion}

The most prominent caveat in our understanding of MS is the primary cause that provokes autoimmunity against the CNS. It is rather hard to detect the seeding changes within or outside the CNS that start the disease pathogenesis. However, this is essential for designing curative MS therapy and preventive tests that would enable early diagnosis of subjects who are at risk of developing MS, that is, subjects who have genetic predisposition for MS. At the moment, there is no combination of biological markers that can predict MS development. The earliest time at which patients can be diagnosed for MS is during the first clinical episode, that is, CIS, which best correlates to a newly developed focal lesion(s) (18). There are not many reports on initial lesions, but most of them concur that their hallmark is the development of ODC death before myelin degradation. This is further accompanied by macrophage-mediated myelin removal, BBB breakage, and potential inflammation (381). Pertinent to this, McFarland and Martin proposed that the initial steps in lesion development can be independent of immune cells (302). This represents a vice versa set of events compared with the processes taking place in the lesions that involve the autoimmune component during MS progression. In those lesions, $\mathrm{T}$ cells, which are autoreactive toward myelin-based epitopes, decompose 
myelin, which, as a consequence, leads to ODC death (33). Studying new symptomatic lesions in patients who died during or shortly after a relapse, Barnett and Prineas observed extensive ODC apoptosis and the presence of reactive astrocytes in early lesions showing generally intact myelin (32). There were also a number of myelin-laden macrophages present, most likely originating from perivascular space or microglia, while lymphocytes were absent. It has been suggested that phagocytic activity in nascent MS lesions represents a response to pathological alternations on myelin sheets developing due to a loss of metabolic support by ODC (33). The authors suggested that these early events in lesion initiation may be followed by the immigration of activated lymphocytes. In another study, Prineas' group documented virtual absence of $\mathrm{T}$ and $\mathrm{B}$ cells in early lesions. They observed ODC loss and the presence of myelin-laden macrophages, which are activated by the presence of degenerate myelin. It has been speculated that the formation of early lesions "has some basis other than destructive cell-mediated immunity directed against a myelin or ODC antigens," while subsequent accumulation of innate immune system cells has been recognized as a response to the presence of dead myelin (194). It is important to note that although ODC death in early lesions has been loosely labeled as apoptosis, it is not apoptosis in its classic form. Namely, an integrative component of the apoptosis pathway-caspase-3-is not active (32). This characteristic, accompanied by DNA condensations, may qualify ODC death in initial lesions as apoptosis-like programmed cell death. The death of ODC via this pathway rarely takes place in chronic MS lesions, implying that it represents an early event which is associated with acute lesion formation $(32,33)$. In an autopsy study, a specific type of lesion (marked as "type I") has been observed almost exclusively in the earliest MS cases (153). The lesions were formed around vessels, and the number of cells was not increased, implying that there was no inflammation; they were also rich in macrophages enclosing myelin material. Probably the most interesting feature was the lack of BBB disruption. In brief, initial white matter lesions show no evidence of T- or B-cell infiltration. Only CNS innate immune system cells - macrophages and activated microgliaare present. According to these facts, the root cause of MS is not immune deregulation, but a set of very specific degenerative processes (417), which, as we will illustrate here, are tightly, if not predominantly, related to redox alterations.

In their milestone study, Lassmann and co-workers determined the presence of four different patterns in MS lesions (283). Patterns I and II involve the autoimmune component and resemble EAE, while patterns III and IV do not comprise autoimmunity, at least not in the early stages of lesion development (Fig. 5). Pattern III lesions appear to be the first to develop. The authors identified pattern III lesions mostly in patients who had a clinical course of less than 8 weeks before the biopsy or autopsy took place (283). Pattern III lesions are localized near the veins or venules. They are characterized by ODC death preceding demyelination, and they show increased expression of redox active enzymes (33). Pattern IV lesions, which are mostly related to degenerative processes and significantly less to inflammation (417), were found in white matter autopsy samples from patients who suffered from PPMS $(283,366)$. Haider et al. labeled pattern II lesions as complement-associated demyelination, whereas pattern III was described as hypoxia-like tissue injury (181). Several groups proposed that pattern III and IV lesions resemble initial starter lesions $(33,259,283)$. In line with this, apoptosislike ODC death was observed before demyelination in pattern III lesions in autopsy samples of MS patients who died shortly after MS development (some less than a month after). T-cell infiltration was mild until serious demyelination developed, which was followed by a massive infiltration (292). In order to integrate the processes involved in MS initiation, we will rely not only on the scarce data on initial/early lesions, but also on what is known about pattern III and IV lesions.

\section{B. Circulatory abnormalities in pre-MS brain}

In order to understand what sets off the formation of initial lesion(s), we should go back to the time before a patient experiences the first signs of MS. A widespread characteristic of MS patients, which appears to be present before the disease onset, is the obstruction of cerebral circulation. A significant role of circulatory defects in MS initiation is implicated by the fact that early lesions (and lesions in general) very frequently develop around veins. For example, Ge et al. documented, using 7T magnetic resonance imaging (MRI), that most lesions form around veins, showing strict perivascular distribution, and following the orientation and course of the vessel (156). Recently, Zamboni and others endorsed and updated so-called chronic cerebrospinal venous insufficiency (CCSVI) hypothesis of MS development, which was proposed by Putnam in 1933 (487). CCSVI describes a vascular condition which is characterized by anomalies of the main extracranial cerebrospinal veins that interfere with normal cerebrospinal outflow, leading to retrograde blood flow. Accordingly, this provokes the lysis of erythrocytes and the formation of "iron sludge," which activates inflammation, thus contributing to MS pathology (487). However, a number of established research groups found strong evidence against CCSVI (456). For example, Doepp et al. found that only a small population of MS patients has CCSVI according to Zamboni's criteria, and that a large majority of those with CCSVI show no measurable hemodynamic alterations (117). In addition, the absence of increased iron levels in patients with CIS clearly implies that iron accumulations do not precede the development of MS and most likely represent an epiphenomenon (236). Finally, a subgroup of adult and pediatric patients showing MS signs that appear to be provoked by iron deficiency has been identified. The symptoms are usually alleviated by iron supplementation (446). This can not only be easily explained by the high amount of iron needed for normal ODC development and function (159), but also substantiates the fact that iron excess is not responsible for MS initiation. In a nutshell, CCSVI is not a plausible cause of MS. However, CCSVI should not be confused with anomalies in cerebral blood flow and small veins and venules inside CNS tissue, as accumulating data foster further concepts, which involve vascular changes and obstructed circulation in MS initiation $(106,456)$. Varga et al. reported that cerebral blood flow is decreased in the normally appearing white matter (NAWM) of patients with CIS compared with control subjects (449). Sinnecker and colleagues documented, using 7T MRI, a reduced visibility of periventricular veins in CIS and early MS patients. The authors attributed the results to hemodynamic and vascular alterations (404). In a very recent MRI study, involving 30 CIS patients, Papadaki et al. observed significantly higher values 
of cerebral blood volume and mean transit time, and decreased cerebral blood flow in NAWM and deep gray matter in CIS compared with healthy subjects. The fact that such changes were not present in RRMS patients implies that they are not secondary (357). Pertinent to this, it has been shown that active lesions in RRMS patients show increased perfusion, whereas inactive lesions show variable levels of perfusion and veins which are ill defined (155). In short, these facts clearly imply that obstructed venous blood flow may be present before the onset of MS. On the other hand, Adhya and co-authors documented reduced cerebral blood flow and blood volume in PPMS patients (2). Another group found a decrease in cerebral blood flow by roughly $50 \%$ and more than twofold prolonged mean transit time of blood throughout the NAWM of MS patients (261). However, these changes, observed during the course of disease progression, could be secondary. It is worth mentioning that epidemiological data seem to concur with the proposed important role of circulatory problems in MS initiation: (i) MS shows similar epidemiological features to chronic vein insufficiency, such as higher prevalence in women and in the white non-Hispanic population compared with Hispanic, African-American, and Asian, very low incidence in aboriginal populations of Africa and Australia $(42,81,100,108,320,372)$, and a similar age of onset (20-30 years of age) $(81,106,372)$; (ii) A decreased blood level of vitamin $\mathrm{D}$, which has been related to an increased risk of MS development (127), may also lead to hypertension and other vascular problems (364). Interestingly, Eskimos and Lapps, who have a rich source of vitamin D in their diet, not only show a low risk of MS development (173), but also rarely develop vascular diseases (433); and finally (iii) one large survey has shown that veterans with MS have a higher prevalence of hypertension compared with the general veteran population (260). However, more correlation studies are needed in order to establish the link proposed here.

\section{Blood-brain nitric oxide crosstalk}

Altered cerebral perfusion may exert a detrimental impact on brain physiology. At the points of blood flow obstruction,
FIG. 5. Four histopathological subtypes of MS lesions. Histopathological description and proposed pathogenic mechanisms are shown under the corresponding illustrations. Mf, macrophage; PC, plasma cell.

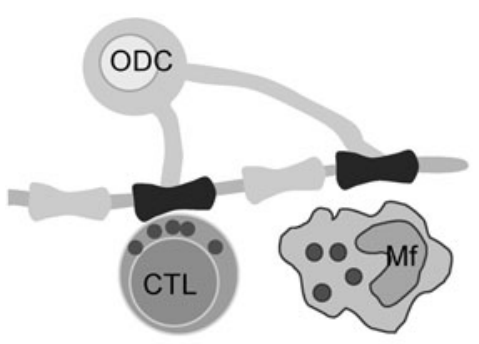

Pattern I

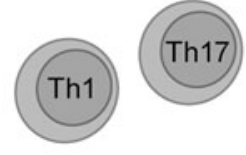

\section{Macrophage mediated}

Perivenous distribution of lesions

Radial expansion of the lesions

Inflammatory infiltrates composed of macrophages and T cells

Activated macrophages and microglia associated with degenerating myelin

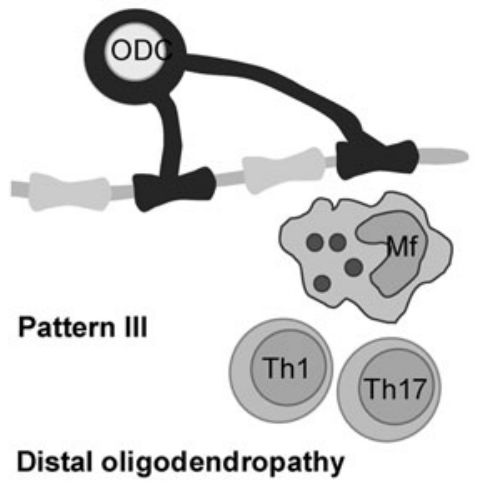

ODC death and the degeneration of distal processes, followed by inflammation mediated by $T$ cells and macrophages
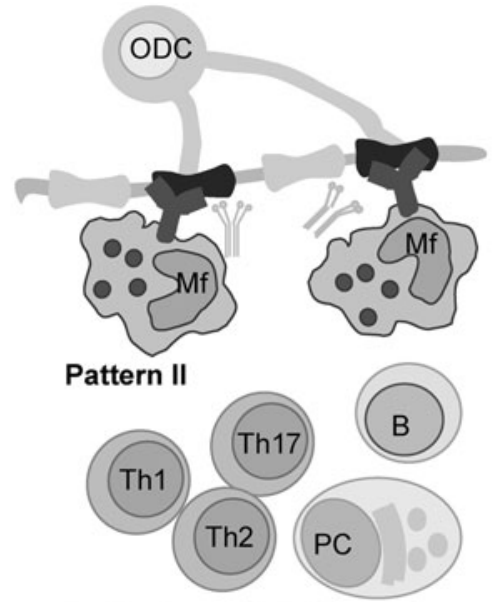

Antibody mediated

Similar lesions as in I but additional deposition of immunoglobulin and activated complement at sites of active myelin destruction

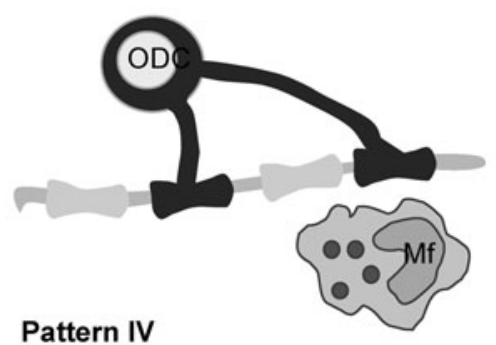

Primary oligodendrocyte damage with secondary demyelination

Prominent ODC degeneration in a small rim of periplaque white matter, followed by pattern I-like processes 
shear stress inevitably develops, being reciprocally proportional to the flow $(85,252)$. This may represent the basis of crosstalk between the circulation and the brain through undisrupted BBB. In response to the shear stress, endothelial cells produce increased amounts of vasoactive agents-nitric oxide (NO) and hydrogen peroxide $\left(\mathrm{H}_{2} \mathrm{O}_{2}\right)(189,277)$. These two reactive species are uncharged and relatively stable, thus being capable of crossing cellular membranes. From the endothelium, $\mathrm{NO}$ and $\mathrm{H}_{2} \mathrm{O}_{2}$ may leak into the perivascular space to affect astrocytes, the extensions of which are positioned around the vessels (Fig. 6). The smooth muscle layer is thick and non-homogenous around small postcapillary veins, and it does not represent a significant barrier for $\mathrm{NO}$ and $\mathrm{H}_{2} \mathrm{O}_{2}$ to reach astrocytes. $\mathrm{NO}$ can be synthesized by three distinct types of NO synthase (NOS): endothelial NOS (eNOS), inducible NOS (iNOS), and neuronal NOS. Generally, eNOS activity in endothelial cells results in micromolar concentrations of $\mathrm{NO}$, which might have only physiological

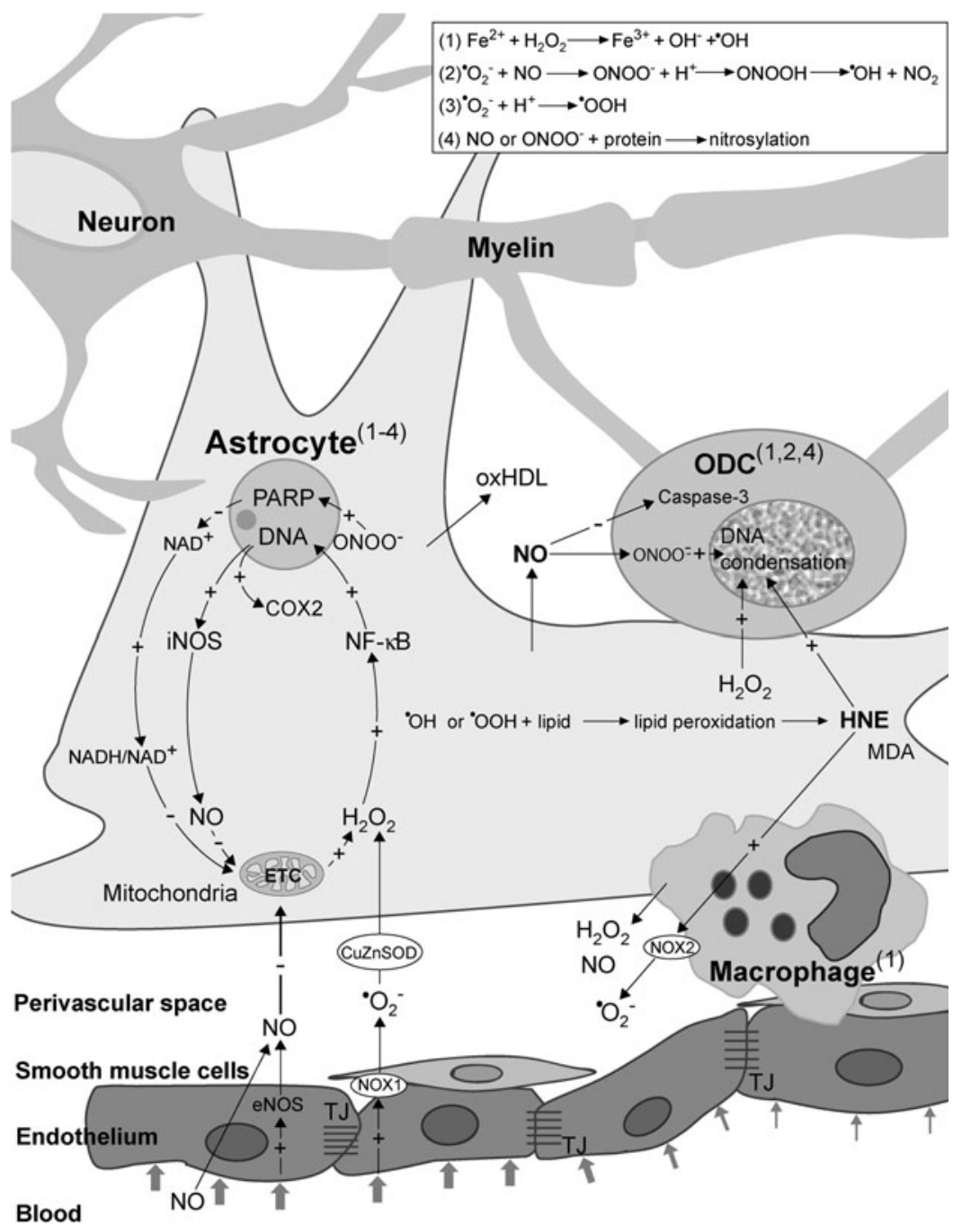

FIG. 6. Redox mechanisms in MS initiation. Obstructed blood flow in narrow vessel results in shear stress, which promotes the production of $\mathrm{NO}$ and ${ }^{\bullet} \mathrm{O}_{2}{ }^{-}$in endothelial cells. Superoxide is dismutated to $\mathrm{H}_{2} \mathrm{O}_{2}$ by CuZnSOD. NO (from endothelium and circulation) and $\mathrm{H}_{2} \mathrm{O}_{2}$ enter astrocytes to activate two redox loops, which intersect in mitochondria. NO inhibits ETC, which results in increased production of $\mathrm{H}_{2} \mathrm{O}_{2}$, and consequent NF- $\kappa$ B activation and iNOS and COX2 expression. iNOS generates large amounts of NO, resulting in further ETC inhibition, thus closing the first loop. ONOO provokes DNA damage, PARP activation, and an increase in $\mathrm{NADH} / \mathrm{NAD}^{+}$ratio, which results in promoted ${ }^{\bullet} \mathrm{O}_{2}{ }^{-}$and $\mathrm{H}_{2} \mathrm{O}_{2}$ production in mitochondria, thus closing the second loop. NO may also provoke macrophage/microglia activation, resulting in increased release of $\mathrm{H}_{2} \mathrm{O}_{2}$ and $\mathrm{NO}$ from these cells. Prooxidative conditions lead to lipid peroxidation, and the production of $\mathrm{HNE}, \mathrm{MDA}$, and oxHDL. NO, HNE, and $\mathrm{H}_{2} \mathrm{O}_{2}$ leak from astrocytes and macrophages and provoke ODC death. An increased level of $\mathrm{NO}$ provokes caspase-3 inhibition and $\mathrm{ONOO}^{-}$formation in ODC. HNE provokes the activation of NOX2 in macrophages. A set of side-reactions (1-4; in the box) takes place in astrocytes, ODC, and macrophages, as marked by superscripts. Reaction (1) is known as the Fenton reaction. The plus (+) and minus (-) symbols represent positive and negative effects on concentration, gene expression, or activity. COX2, cyclooxygenase 2; CuZnSOD, copper, zinc superoxide dismutase; ETC, electron transport chain; $\mathrm{H}_{2} \mathrm{O}_{2}$, hydrogen peroxide; $\mathrm{HNE}$, 4-hydroxy-2-nonenal; iNOS, inducible nitric oxide synthase; MDA, malondialdehyde; NF- $\kappa$ B, nuclear factor-kappaB; ODC, oligodendrocytes; oxHDL, oxidized high-density lipoproteins; PARP, poly (ADP-ribose) polymerase. 
effects (266). However, the effects may transcend pathophysiological ones, if the NO concentration exceeds a specific threshold. This may occur in the case of an increased level of NO or its precursor-nitrite-in the systemic circulation. Such a setup may be developed in a potential MS patient due to infection- or injury-related inflammation at remote sites or due to increased core body temperature $(234,382)$. Pertinent to the later, Uhthoff's phenomenon, the manifestation of MS symptoms and signs when core body temperature is increased by exercise or a hot bath, has been used for decades in MS diagnostics (90). It is important to note that a recent study showed that eNOS expression is, in fact, decreased in early lesions (140). However, one should bear in mind that increased expression of eNOS provoked by shear stress represents a transient effect, which develops before the development of the lesion and is further attenuated by increased NO concentrations (189). The important role of eNOS in MS initiation is implicated by the fact that eNOS-deficient mice exhibit a delayed onset of EAE, which correlates with delayed BBB breakdown (467). Shear stress also increases the activity of NADPH oxidase 1 (NOX1) on endothelial cell membranes (150), which leads to superoxide $\left({ }^{\bullet} \mathrm{O}_{2}{ }^{-}\right)$production. Fischer et al. reported that this setup develops in initial lesions (140). Since CSF is rich in copper, zinc superoxide dismutase (CuZnSOD) (256), the expression of which may be further promoted by shear stress (189), NOX1-mediated ${ }^{\bullet} \mathrm{O}_{2}{ }^{-}$generation results in the rise of extracellular concentrations of $\mathrm{H}_{2} \mathrm{O}_{2}$.

\section{D. $\mathrm{NO}$ and $\mathrm{H}_{2} \mathrm{O}_{2}$ from endothelium activate the redox cycle in astrocytes}

$\mathrm{NO}$ and $\mathrm{H}_{2} \mathrm{O}_{2}$ are capable of drastically altering the intracellular redox milieu and of setting a cascade of events that may result in cellular dysfunction or death (15). A number of studies substantiate the development of redox changes in MS lesions, involving nitrosative and oxidative stress. It is particularly interesting that the production of reactive oxygen and nitrogen species (ROS and RNS) in active MS lesions is generally increased only in specific cells, namely astrocytes, macrophages, and endothelial cells, all of which have a direct contact with perivascular space $(275,347,444)$, as well as in ODC (181). NO may provoke the inhibition of mitochondrial electron transport chain (ETC) complexes I, III, and IV, leading to increased ${ }^{\bullet} \mathrm{O}_{2}{ }^{-}$production (333). Mitochondria represent the main site of production of ${ }^{\bullet} \mathrm{O}_{2}{ }^{-}$and $\mathrm{H}_{2} \mathrm{O}_{2}$. The production takes place even under physiological conditions, being drastically increased if ETC is obstructed (333). There are no data on the activity of complexes I and III in early MS lesions, but Mahad et al. observed the inhibition of complex IV in astrocytes and ODC in pattern III lesions, which could be a result of the supraphysiological NO level. It is important to note that inhibition was not present in pattern II lesions (288). The increased production of ${ }^{\bullet} \mathrm{O}_{2}{ }^{-}$and its derivative $\mathrm{H}_{2} \mathrm{O}_{2}$ in astrocytic mitochondria is implicated by the increased levels of mitochondrial antioxidative enzymes-manganese superoxide dismutase (MnSOD) and peroxiredoxin $\mathrm{V}$-in active lesion astrocytes and NAWM $(203,444)$. Finally, it would have been expected that the inhibition of mitochondrial respiration leads to decreased $\mathrm{O}_{2}$ utilization, and exactly this was reported to develop in the white matter of MS patients (157).

In contrast to ${ }^{\bullet} \mathrm{O}_{2}{ }^{-}$, which is charged, incapable of crossing mitochondrial membranes, and barely capable of crossing cellular membranes (via $\mathrm{Cl}^{-}$channels), $\mathrm{H}_{2} \mathrm{O}_{2}$ can easily enter the cytosol from mitochondria and perivascular space, where it can activate nuclear factor-kappa B (NF- $\kappa$ B) (Fig. 6) (15). Pertinent to MS, Bonetti et al. observed the activation of NF- $\kappa \mathrm{B}$ in astrocytes at the edges of active lesions (52). The importance of NF- $\kappa \mathrm{B}$ activation in astrocytes is strongly substantiated by the study performed on the cuprizone model of demyelination (371). The authors reported that the depletion of $\mathrm{I} \kappa \mathrm{B}$ kinase (which is essential for NF- $\kappa$ B activation) in astrocytes, but not in ODC, was sufficient to protect the mice from myelin loss. NF- $\kappa$ B activates the expression of a number of genes, two of which encode redox active enzymes-iNOS and cyclooxygenase 2 (COX2, which has ${ }^{\bullet} \mathrm{O}_{2}{ }^{-}$as a by-product) (15). The increased level of astrocytic iNOS has been recognized as an important pathogenic feature of MS (318). Under normal settings, the level of active iNOS within the CNS is next to zero. The activation of its expression leads to a drastic increase in NO generation, resulting in millimolar NO concentrations. It is important to note that $\mathrm{NO}$ reacts very fast with ${ }^{\bullet} \mathrm{O}_{2}{ }^{-}$to produce peroxynitrite $\left(\mathrm{ONOO}^{-}\right)$, which can exert direct negative effects or can be decomposed to dangerous hydroxyl $\left({ }^{\circ} \mathrm{OH}\right)$ and nitrogen dioxide radicals $\left({ }^{\bullet} \mathrm{NO}_{2}\right)(15)$. $\mathrm{ONOO}^{-}$and - $\mathrm{NO}_{2}$ may react with Tyr residues in proteins to produce nitrotyrosine. Pertinent to this, Oleszak et al. observed increased levels of iNOS and nitrotyrosine in astrocytes and macrophages in acute monophasic lesions, while this was not the case in chronic lesions (347). Similar results were acquired in other studies, reporting that astrocytes and endothelial cells are positive for nitrotyrosine in active lesions (101, 275). The authors have proposed that astrocyte-derived NO could be important for orchestrating further processes in MS. An abundant expression of iNOS in macrophages early in the course of the disease and before demyelization was also reported (49). The importance of iNOS expression in MS development is further substantiated by the fact that the injection of arginine [which acts both as an iNOS substrate and an up-regulator of iNOS mRNA (263)] into the rat brain provokes demyelination (248). The expression of iNOS in astrocytes most likely represents a key step in MS initiation, leading to further NO-provoked inhibition of ETC and the formation of a self-promoting redox loop (Fig. 6).

\section{E. The decrease of $N A D H / N A D^{+}$ratio}

Probably the most important pathophysiological consequences of the increased NO level are indirectly provoked by $\mathrm{ONOO}^{-}$. Peroxynitrite targets DNA and causes single-strand breakage, which leads to poly (ADP-ribose) polymerase (PARP) activation (15). Veto et al. observed strong PARP immunoreactivity in astrocytes and ODC in pattern III lesions in patients with MS duration from 6 to 60 days. PARP activity was at its highest in very early lesions (453). It has been concluded that PARP activation plays a critical role in MS pathogenesis $(136,453)$. The importance of the $\mathrm{NO} \mathrm{ONOO}^{-} /$ PARP pathway is implicated by the inverse correlation between MS activity and CSF and sera levels of uric acid (123), which represents an $\mathrm{ONOO}^{-}$and ${ }^{\bullet} \mathrm{OH}$ scavenger (410). Although the application of inosine (uric acid precursor) did not show significant beneficial effects in an MS trial (170), PARP inhibition has been recently praised as a promising approach in MS treatment $(79,136)$, primarily because it prevents ODC death (453). However, precaution should be taken in 
balancing between positive and negative effects of PARP activation in MS. A complete inhibition of PARP function could result in an inability of cells to repair DNA and may even promote MS. For example, PARP-1 $1^{-/}$EAE mice show an earlier onset and develop a more severe EAE when compared with wild-type animals (396).

While the activation of PARP in ODC in MS lesions represents an echo of DNA fragmentation and cellular death, in astrocytes that do not undergo such changes the activation of PARP may represent an important step in MS pathogenesis. PARP catalyzes the cleavage of $\mathrm{NAD}^{+}$, which leads to a decrease in the $\mathrm{NAD}^{+}$level and an increase in the NADH/NAD ${ }^{+}$ ratio (15). A high $\mathrm{NADH} / \mathrm{NAD}^{+}$ratio is known to act as the strongest stimulus for ${ }^{\bullet} \mathrm{O}_{2}{ }^{-}$production in mitochondria (on ETC complex I) (333). The increased production of ${ }^{\bullet} \mathrm{O}_{2}{ }^{-}$in complex I results in further $\mathrm{H}_{2} \mathrm{O}_{2}$ leakage from mitochondria, thus closing another redox loop (Fig. 6). The development of interdependent redox loops in the course of MS pathogenesis is implicated by the fact that nanomolar affinity PARP inhibitor PJ-34 (which delays EAE onset) suppresses iNOS expression (394).

\section{F. Astrocytes and macrophages: centers of oxidative/ nitrosative stress in early lesions}

In a recent commentary, Smith has proposed that astrocytes are responsible for oxidative/nitrosative damage in ODC and neurons in MS (406). The development of pathophysiological processes that involve positive feedback loops in astrocytes and the activation of macrophages result in these cells becoming the main source of ROS and RNS in early/ pattern III lesions. In line with this, astrocytes and macrophages exhibit the activation of the antioxidative system (AOS) and increased levels of the products of oxidation and nitrosylation $(275,347,444)$. Van Horssen and co-authors observed that in active lesions only astrocytes and perivascular or myelin-laden macrophages show increased levels of CuZnSOD, MnSOD, catalase, NAD(P)H:quinine oxidoreductase 1, as well as nitrotyrosine and 4-hydroxy-2-nonenal (HNE; a product of lipid peroxidation) (444). It is important to note that the increased level of MnSOD was observed only in astrocytic mitochondria (444). This implies that ETC is obstructed only in astrocytes, while macrophages generate ROS in the intracellular compartment and on the membrane (see section III.A). The expression of many AOS enzymes is regulated by nuclear factor-E2-related factor (Nrf2)-antioxidant response element (ARE) pathway, the activation of which in MS is specifically located in astrocytes (443). Newcombe et al. reported the presence of increased levels of HNE in astrocytes and malondialdehyde (MDA; another product of lipid peroxidation) in astrocytes and myelin-laden macrophages in early lesions (341). The importance of oxidative stress in MS initiation is strongly implicated by the results showing that the level of oxidation markers is more than 8-fold higher in early lesion-resembling pattern III lesions than in pattern II lesions (181). One small study has observed increased levels of lipid peroxidation markers in the CSF of probable MS patients (337). This clearly implies that oxidative changes in lipids take place early in MS development (i.e., before MS can be diagnosed for certain). It is rather intriguing that the only lipoprotein particles present in human CSF-high-density lipoprotein (HDL)-like particles_-are specifically secreted by astrocytes and macrophages/microglia (138). It is plausible that oxidized HDL-like particles (oxHDL) could be released from astrocytes and macrophages under prooxidative settings in initial lesions. The particles may integrate into membranes of other cells in order to activate a lipid peroxidation-chain reaction (15), thus disseminating oxidative damage. It has been reported that oxHDL exerts toxic effects on cultured CNS cells and promotes inflammation (138); so, these structures may be involved in the mechanisms of initial lesion formation as well as in disease progression. It is worth mentioning that HDL comprises antioxidative enzymes, the activity of which is decreased by excessive oxidation (138).

The involvement of macrophages/microglia in the development of the initial lesion is strongly implicated by the available data. These cells could promote prooxidative conditions in initial lesions after they recognize cell injury, but the activation may take place even before that. Namely, NO released by endothelial cells and astrocytes appears to act as an independent signal for macrophage/microglia activation (92). Exogenous NO (not produced in macrophages) has been reported to: orchestrate macrophage/microglia migration and accumulation, acting as a chemoattractant $(84,115,415)$, induce microglia recruitment to the lesion $(115,122)$, provoke morphological changes of microglia into ameboid-like cells (115), and provoke hormesis-the exposure to low level NO increases the resistance of macrophages to pro-apoptotic NO overload (481). Activated macrophages/microglia are known to produce and release $\mathrm{NO}, \mathrm{H}_{2} \mathrm{O}_{2}$, and ${ }^{\bullet} \mathrm{O}_{2}{ }^{-}$(see section III.A.).

\section{G. Oligodendrocytes death}

It has been postulated that the dysfunction of astrocytes precedes ODC degeneration in early lesions (400). $\mathrm{NO}, \mathrm{H}_{2} \mathrm{O}_{2}$, and HNE may leak from astrocytes and activated macrophages in order to affect other CNS cells, ODC in the first place. For example, it has been shown that astrocytes expressing syncytin (human endogenous retrovirus glycoprotein) provoke the death of ODC by producing excessive amounts of NO on iNOS (19). One may ask since astrocytes represent the source of nitrosative and oxidative stress, why they are not dying while ODC do. In a comparative study, Garthwaite et al. found that the rank order of susceptibility of CNS cell to NO is neurons $>$ ODC > astrocytes (152). The experimental design did not allow work on myelinated neurons, which are known to be drastically less susceptible to NO compared with demyelinated cells (378). This implies not only that the myelin sheet provides axons with good protection from $\mathrm{NO}$ excess, but also that the susceptibility under in vivo conditions is, in fact, ODC $>$ (myelinated) neurons $>$ astrocytes. The analysis of biopsy samples obtained from a young patient with early MS revealed the accumulation of nitrotyrosine in ODC, pinpointing it to $\mathrm{ONOO}^{-}$-mediated damage (488). It has been documented that $\mathrm{ONOO}^{-}$does not provoke apoptosis in ODC, and necrosis was proposed to be the main route of cell death (394). On the other hand, astrocytes appear to be relatively resistant to $\mathrm{ONOO}^{-}$(220).

Although ODC are obliged to generate large amounts of ATP in order to maintain high metabolic overturn (424), their sensitivity to NO cannot be explained by NO-provoked ETC inhibition (220). Mature, myelinating ODC successfully survive mitochondrial dysfunction by switching to glycolysis (146). Therefore, NO may act indirectly, by promoting the formation of $\mathrm{ONOO}^{-}$, which is known to provoke DNA 
damage. In addition, ODC are sensitive to oxidative stress, because they have low antioxidant levels, a high content of iron (redox active metal), and exposed extensive plasma membrane elaborations (33). ODC precursors appear to be even more susceptible to oxidative stress and glutathione (GSH) depletion compared with mature cells $(26,144)$. They show thrice lower GSH concentrations and 20 times higher iron level than astrocytes (423). Neurons are also known for their weak AOS, but their axons acquire protection from myelin sheets. For example, Peterson et al. found that the number of apoptotic neurons per $\mathrm{mm}^{2}$ was substantially lower in myelinated than in non-myelinated cortex areas in the MS brain (363). This may explain why an oxidative/nitrosative burst unleashed by the astrocytes leads to ODC death, but leaves neurons generally uninjured. Myelin takes the "blow" for the axons because of the spatial arrangement, and because it is rich in iron and lipids (159), which makes it a perfect buffer for reactive species coming from the surroundings. Iron may bind $\mathrm{NO}$ in order to form dinytrosil-iron complexes (DNIC) (442), or it may react with $\mathrm{H}_{2} \mathrm{O}_{2}$ to produce a highly reactive ${ }^{\circ} \mathrm{OH}$ radical, which is buffered by myelin lipids. It has been shown that ODC in co-culture protect neurons from NO-mediated damage and that this protection is dependent on the function of iron-releasing enzyme heme oxygenase-1 (HO-1) (47). This implies a possible protective role of DNIC formation in CNS against NO overload. Neurons may be affected by $\mathrm{NO}$ only at myelin-unprotected points (nodes of Ranvier). Pertinent to this, NO is known to block the conduction in axons (378), which in some cases may lead to the (first) presentation of MS symptoms.

ODC are particularly susceptible to HNE, a major bioactive lipid peroxidation product, which is diffusible and highly toxic (444). HNE level is increased 4-fold in MS active lesions compared with control tissue (463). The reported HNE concentration $(\sim 4 \mu M)$ appears to be more than sufficient to kill ODC according to the findings of an in vitro study which showed that HNE provokes ODC death at concentrations as low as $2 \mu M$ (151). On the other hand, at this concentration, generally, HNE does not exert significant negative effects on other cells. For example, the first toxic effects on endothelial cells have been observed at $25 \mu M$ (435). In addition to ODC death, the accumulation of HNE may obstruct the remyelination by inhibiting the proliferation and differentiation of ODC precursors (151).

Unfortunately for the patients, ODC in MS do not undergo apoptosis, which is the safest way to die if we consider the safety of surrounding tissue. Although nuclear condensation and fragmentation in ODC have been observed in acute MS lesions, caspase-3, which is the main effector of classic apoptosis, was not found to be active $(1,32)$. This can be attributed to the fact that NO acts as a strong inhibitor of caspase-3 (239). In addition, it has been suggested that $\mathrm{HNE}, \mathrm{NO}$, or $\mathrm{ONOO}^{-}$ do not provoke ODC apoptosis but most likely necrosis or apoptosis-like programmed cell death (301, 322, 394). $\mathrm{H}_{2} \mathrm{O}_{2}$ may also contribute to non-apoptotic death by provoking DNA fragmentation (330).

\section{H. Demyelination}

Myelin membranes exceed 100-fold the weight of ODC (424). After ODC death, this huge amount of membrane becomes metabolically unsupported. It is interesting that in initial lesions, the first myelin abnormalities develop at the inner, most distant (compared with ODC body) part of the myelin sheath (381), which is the first to suffer the lost of metabolic support. In addition, S-nitrosylation of myelin proteins (in particular, PLP) may result in myelin decompaction (50). Consequently, the externalization of phosphatidylserine develops, making this dominant mammalian scavenger signal available to macrophage receptors (33). A number of studies documented the presence of myelin-laden macrophages in early/pattern III lesions, most likely originating from perivascular space or microglia $(32,447)$. The density of perivascular macrophages gradually increases along the vascular tree, from capillaries to postcapillary venules, which represent the most probable site of formation of initial lesions (40). It is worth mentioning that the loss of metabolic support may lead to myelin fragmentation even in the absence of macrophages (32).

Myelin-laden macrophages principally represent an antiinflammatory acting component of the immune system. However, when exposed to specific stimuli, such as pronounced oxidation, their myelin-removing function may become hampered, while the activity may switch from anti- to pro-inflammatory mode (35). It appears that in MS lesions, macrophages get exposed to excessive oxidation, according to the increased levels of catalase, CuZnSOD, and HNE (444). In addition, it seems plausible that macrophages may be paying the price of their own function. Since myelin sheets contain high levels of iron, myelin-laden macrophages in MS lesions accumulate this transition metal (267), which may promote Fenton reaction-mediated oxidation in the intracellular compartment (Fig. 6). The oxidation-provoked shift in macrophage function may have the incomplete removal of myelin debris as a consequence, which could promote the development of autoimmunity.

\section{BBB breakdown}

The outlined set of events may exert a tremendous impact on BBB, provoking its dysfunction and breakage via several different mechanisms. First of all, astrocyte foot processes are placed around the blood vessels, contributing to the formation and function of BBB. Astrocytes induce and regulate TJ, balanced redox milieu, high mitochondrial content, and other intrinsic properties of neural endothelial cells that constitute BBB (318). The activation of redox processes resulting in ETC inhibition (and consequent ATP shortage) and pronounced oxidation/nitrosylation may lead to the dysfunction of astrocytes. Such astrocytes may not be capable of properly supporting the integrity of BBB at the site of lesion formation. In addition, altered redox settings may lead to a release of a number of TJ/BBB-affecting agents into perivascular space (242), such as matrix metalloproteases (MMP), $\mathrm{HNE}, \mathrm{H}_{2} \mathrm{O}_{2}$, $\mathrm{NO}$, and ${ }^{\bullet} \mathrm{O}_{2}{ }^{-}$(Fig. 7). The stability of endothelium-astrocyte configuration requires matrix adhesion, which may be altered by MMP (113). The expression of MMP-2 and -9 has been detected in astrocytes in MS lesions (102), and it is most likely related to COX2 activity (74). In addition, exposing the endothelial cells and smooth muscles to $\mathrm{H}_{2} \mathrm{O}_{2}$ or ${ }^{\bullet} \mathrm{O}_{2}{ }^{-}$is known to promote MMP-2 activity $(43,437)$, whereas HNE is known to provoke increased MMP-9 release from macrophages (264). Shear stress is known to induce an increase in the level of MMP-2 and -9 in venous tissue (359). Finally, the exposure of 


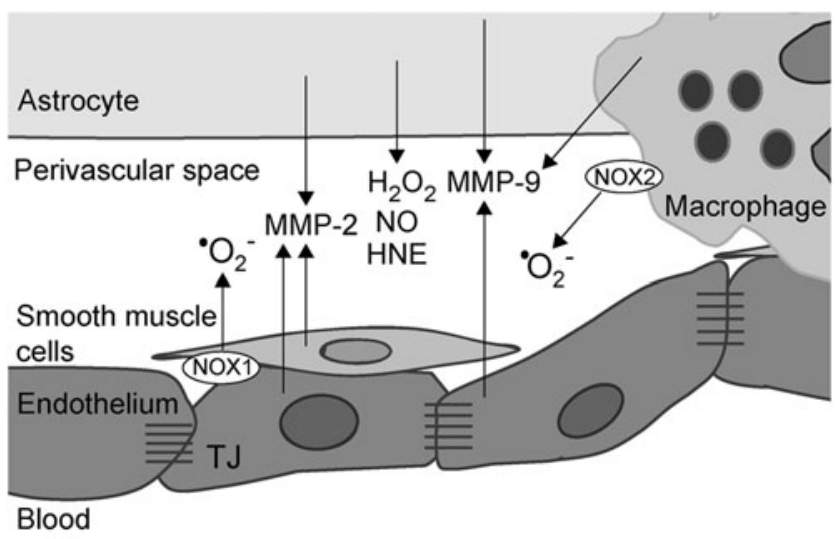

FIG. 7. The release of TJ/BBB-affecting agents into perivascular space in MS. Prooxidative conditions and shear stress result in the release of a number of compounds into perivascular space: MMP-2 and -9, NO, $\mathrm{HNE}, \mathrm{H}_{2} \mathrm{O}_{2}$, and $\mathrm{O}_{2}{ }^{-}$, all of which may affect TJ and BBB. The potential sources (cell types) of these species are presented. MMP, matrix metalloproteases. endothelial cells to MBP may affect BBB, and it may promote the production of MMP-2 (105).

Figure 8 shows TJ before and after the attack of a set of agents that are involved in MS pathogenesis. MMP-2 and MMP-9 cleave occludin and dystroglycan receptors, which leads to the destabilization of TJ and astrocyte end-feet anchorage to parenchymal membrane $(4,163)$. In addition, MMP participate in the degradation of collagens and elastin (290). This results in the formation of different protein fragments, some of which may act as chemoattractants or immunomodulators, for example, the cleavage of collagen may produce bioactive tripeptide Pro-Gly-Pro, which recruits neutrophils (457). HNE affects the permeability of endothelium by changing the distribution of cadherin, $\beta$-catenin, and zonula occludens 1 (ZO1), and by causing a decrease in the surface integrin. In addition, HNE provokes dissolution of peripheral actin bands and the formation of actin aggregates in the cytosol. These and some other changes result in disturbed cell-cell adhesion contacts, intercellular gap formation, and transendothelial cell migration (435). At least some of these effects may be attributed to HNE-provoked ROS production by the mitochondria in endothelial cells and by
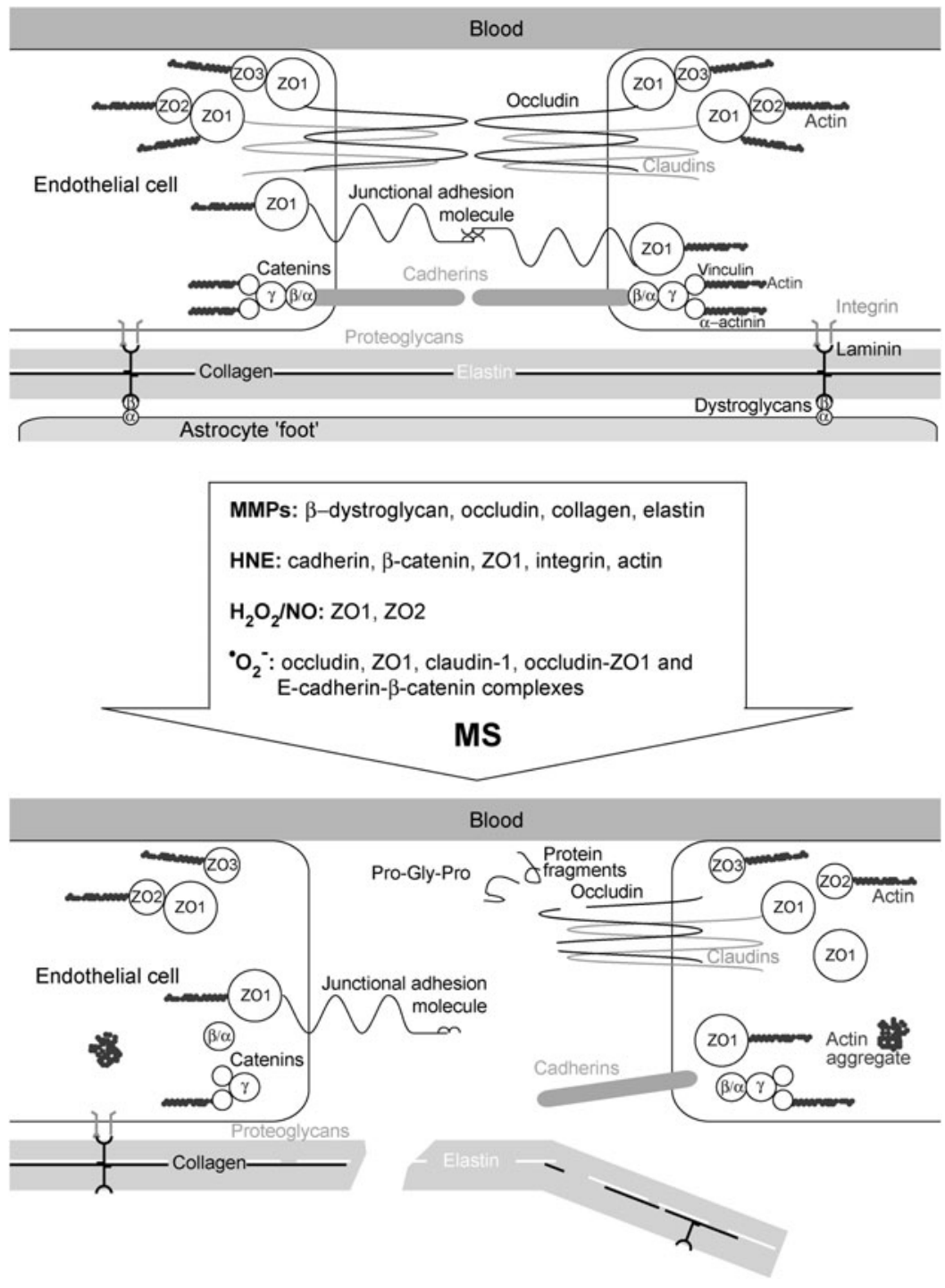

FIG. 8. Structural alternations in TJ provoked by redox changes in MS. A set of agents-MMP-2 and -9, NO, $\mathrm{HNE}, \mathrm{H}_{2} \mathrm{O}_{2}$, and ${ }^{\bullet} \mathrm{O}_{2}{ }^{-}$- which are released into the perivascular space from astrocytes, endothelium, and macrophages, impact different components of TJ (enlisted within the arrow). This provokes the TJ (top image) to lose its structure and integrity (bottom image). MMP-2 and MMP-9 cleave occludin and dystroglycan receptors, which leads to the destabilization of TJ and astrocyte end-feet anchorage to parenchymal membrane. MMP-mediated cleavage of collagens and elastin results in the destruction of lamina, and potentially in the release of some chemoattractants (e.g., Pro-Gly-Pro). HNE changes the distribution of cadherin, $\beta$-catenin, and ZO1, and causes dissolution of peripheral actin bands and the formation of actin aggregates in the cytosol. $\mathrm{H}_{2} \mathrm{O}_{2}$ affects $\mathrm{BBB}$ by changing the localization of $\mathrm{ZO} 1$ and $\mathrm{ZO} 2$, the effects being, most likely, mediated by increased NO production. Superoxide suppresses the expression of junction adhesion molecules, ZO1, claudin-1, and occludin, and provokes the dissociation of occludin-ZO1 and E-cadherin- $\beta$-catenin complexes. ZO, zonula occludens. 
NOX2 in macrophages $(435,483)$. It has been shown that $\mathrm{H}_{2} \mathrm{O}_{2}$ affects $\mathrm{BBB}$ by changing the localization of $\mathrm{ZO} 1$ and $\mathrm{ZO} 2$ (262). Consistent with this, $\mathrm{H}_{2} \mathrm{O}_{2}$-removing enzymescatalase and GSH peroxidase-are known to protect BBB from disruption (178). $\mathrm{H}_{2} \mathrm{O}_{2}$ has been shown to increase solute permeability of endothelium, the effects being pronounced by the co-supplementation of a NO donor, and completely suppressed by NOS inhibition (346). This implies that the mechanisms by which $\mathrm{H}_{2} \mathrm{O}_{2}$ affects endothelial permeability overlap or depend on NO-provoked changes. Peroxynitrite may also be involved, as the application of $\mathrm{ONOO}^{-}$scavenger prevented $\mathrm{BBB}$ permeability changes in an EAE model (206). Finally, ${ }^{\bullet} \mathrm{O}_{2}{ }^{-}$may also have its place in BBB disruption. Huppert and colleagues postulated that IL-17-induced $\mathrm{BBB}$ disruption involves NOX- and xanthine oxidase (XO)mediated ${ }^{\bullet} \mathrm{O}_{2}{ }^{-}$production and consequent down-regulation of occludin (213). The intracellular ${ }^{\bullet} \mathrm{O}_{2}{ }^{-}$production in endothelial cells appears to suppress the expression of junction adhesion molecules, ZO1, claudin-1, and occludin (476). Pertinent to this, TJ in the brain of MS patients shows a lower level of expression of junction adhesion molecule A (353). Rao et al. showed that extracellular production of ${ }^{\bullet} \mathrm{O}_{2}{ }^{-}$induces tyrosine phosphorylation of occludin-ZO1 and E-cadherin$\beta$-catenin complexes, resulting in their redistribution, dissociation (376), and, finally, in TJ disruption (Fig. 8). It should be noted that TJ of postcapillary brain venules are less complex than those of brain capillaries, which is probably one more attribute that helps the development of lesions around venules.

The process of MS initiation might stop right here. No autoimmune response has to develop. A specific part of CNS suffered demyelination, which can be reversible, and the patient may go on with his/her life for years, without experiencing further disabilities. This benign MS appears to develop in approximately $30 \%$ of MS patients (365). However, in many patients, the process transcends to the next phase, which involves antigen formation and the development of autoimmunity. Pertinent to this, it has recently been proposed that MS pathogenesis involves primary degenerative changes that may (or may not) result in a host's aberrant immune response (a so-called inside-out model of MS) (417). A gate opens to the damaged tissue emitting specific signals and showing epitopes that the immune system is not familiar with, inviting the invasion of immune cells and ending up in MS taking its inflammatory/autoimmune course, as we will discuss in the following section. It should be emphasized that MS may take a benign course even after the development of the autoimmune component $(94,284)$.

\section{J. The development of autoimmunity}

1. Antigens. A combination of ODC's non-apoptotic death and macrophage dysfunction may have a crucial influence on the further steps in MS initiation, that is, induction of autoimmunity. Non-apoptotic and hence not fully controlled death of ODC may go hand in hand with macrophages and microglia's decreased capability of removing myelin, resulting in the accumulation of myelin fragments and vesicles at the site of an early lesion $(153,191)$. It has recently been reported that in a homeostatic process, microglial cells constantly take up exosomes from ODC, which are unable to recycle membrane components from myelin sheaths that are distant from their somas (141). As a consequence of inflammation, microglial assistance to the recycling is reduced, and myelin-filled exosomes in interstitial fluid might reach CSF (375). Pertinent to this, recognizable myelin fragments have been frequently detected in CSF sediments taken at the time of acute MS attacks (196). It is noteworthy that the phagocytosis of necrotic cells promotes the maturation of DC (389).

The presence of not one but a number of different products of myelin that become antigens in MS is substantiated by the work of Zhang et al. (491) and Bahbouhi et al. (28). They showed that there is a population of $\mathrm{T}$ cells memorizing a wide-spectrum of myelin-related antigens in the blood of MS patients. This is in line with the recent assertion that, instead of the classic "single-epitope-targeting" approach, a "multiepitope-targeting" strategy is required for effective immunespecific therapy for MS (230). A multi-epitope setup may explain why several decades of effort failed at identifying one (or few) specific antigens that are responsible for the autoimmune component of MS pathophysiology. It is interesting that a number of reactive species and products of oxidation in MS lesions may participate in the formation of epitopes (Fig. 9). MDA may form an adduct with MOG, which is taken up more effectively by APC than MOG. In addition, the exposure of mice to MDA-MOG led to increased expression of IL-23, IL-12, and IL-12R in APC, resulting in differentiation of Th17 and Th1 cells (454). Pertinent to this, CSF of MS patients shows increased presence of MDA and MDA adducts on lysine residues in proteins and increased levels of antibodies for MDA-low-density lipoproteins (LDL) compared with controls $(161,172)$. HNE forms stabile adducts with lysine, histidine, or cysteine (Cys) residues in proteins, which are accumulated in MS lesions (463), and may elicit a strong immune response (7). Some other epitopes, derived from lipid peroxidation, may also act as pathogenic factors in MS. In a recent study, Gonzalo and colleagues found increased levels of carboxymethylated and neuroketal-modified proteins in the CSF of MS patients. The level of neuroketal-modified proteins showed a positive correlation with the amount of antibodies against lipid peroxidation-modified proteins in $\mathrm{CSF}$, which implies that this adduct may have a role of antigen in MS (172). The presence of anti-SNO-Cys antibodies that target S-nitrosylated proteins was documented in the sera of MS patients and in an EAE model (54). Duleu et al. documented an increased presence of circulating antibodies directed against a variety of $\mathrm{NO}$ - and $\mathrm{ONOO}^{-}$-modified ( $\mathrm{NO}_{2}$-tyrosine) self-antigens in MS patients (124). Nitrotyrosine residues showed the ability to evade central tolerance and to elicit a robust T-cell immune response (46). Discerning whether, when, and which myelin products are to be recognized as antigens, in combination with the characteristics of T-cell populations that target myelin products, may represent the key for development of different MS subtypes. It is important to note that many neurodegenerative diseases are associated with the release of nervous tissue-based antigenic structures, yet the patients do not show signs of MS. It has recently been shown that ODC death (induced by diphtheria toxin in diphtheria toxin receptor-expressing ODC) does not elicit anti-CNS immunity even though the fragments of myelin have been detected in CNS-draining lymph nodes, and not even when the immune system is strongly prestimulated (279). This is in line with the previous assertion that ODC death does not provoke an immune response in multiple system atrophy (461). These findings strongly imply that the fragments of nervous tissue or myelin are not sufficient to 
provoke an autoimmune response. It appears that some MSspecific myelin-based highly-antigenic molecules, such as MDA-MOG, HNE- and neuroketal-adducts, nitrosylatedpeptides, or citrullinated-MBP (citrulline being the product of iNOS activity) $(172,417)$, are necessary in order to push MS over the edge of autoimmunity.

2. The activation/reactivation of T cells. The initiation of autoimmunity is a consequence of an efficient presentation of CNS antigens to T cells by DC in lymphoid organs (Fig. 9A). DC may collect the antigen within the CNS and then go to the lymph node, or they may reside in the node and trap the
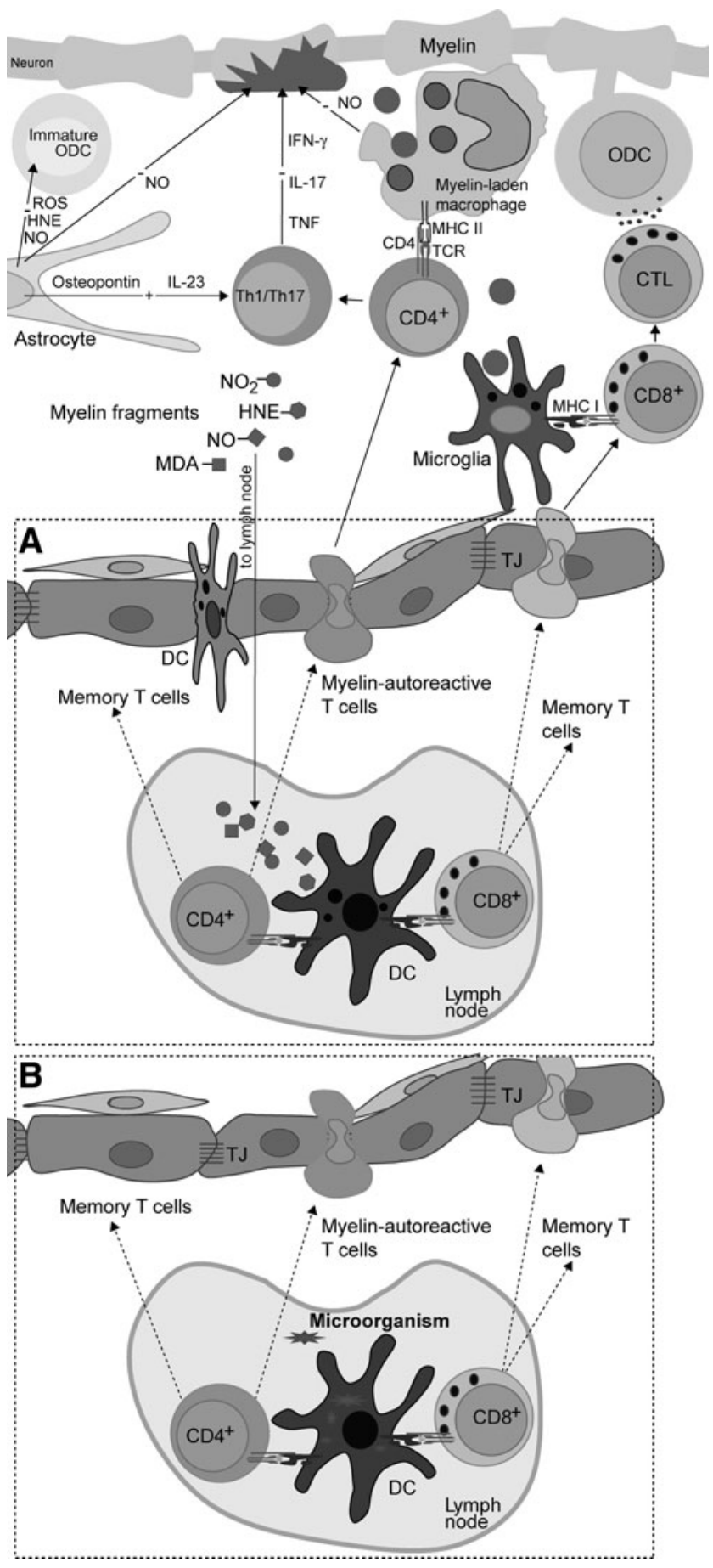

antigens in the lymph draining from the CNS. It seems plausible that both, the migration of DC and the leakage of soluble antigens from the CNS, could be facilitated in the case of BBB or BCSFB breakage. DC present antigens in the form of peptides in the context of MHC class II molecules to naïve $\mathrm{CD}^{+} \mathrm{T}$ cells. The presentation of antigens by $\mathrm{DC}$ to naïve autoreactive $\mathrm{CD}^{+} \mathrm{T}$ cells and their reactivation in CNS by microglia depends on the process of antigen cross-presentation. Generally, molecules of MHC class I present intracellular antigens, while molecules of MHC class II present ingested antigens. In order for APC to present an ingested antigen to $\mathrm{CD}^{+} \mathrm{T}$ cells, it has to engage MHC class I molecules. Antigen processing and presentation by MHC class I and MHC class II molecules are spatially distinct in cells. However, leakage of antigens processed by MHC class II machinery into MHC class I pathway occurs in the process of cross-presentation (310). Activated T cells will differentiate into effector and memory $\mathrm{T}$ cells.

After initial activation, $\mathrm{T}$ cells migrate into the CNS, where they are reactivated. As a consequence of redox-imposed damage to nervous tissue, CNS-resident phagocytes are activated in order to present antigens on MHC class II molecules. Autoreactive $\mathrm{CD}^{+}{ }^{+} \mathrm{T}$ cells are reactivated after interacting with these cells $(34,231)$. After that, they differentiate into effector interferon (IFN)- $\gamma$-producing Th1 cells and/or IL-17-producing Th17 cells (129). The stimulated effector Th cells locally release proinflammatory cytokines, thus

FIG. 9. The development of autoimmunity in MS as a consequence of redox changes in the CNS. ROS and RNS damage myelin and disturb homeostatic functions of astrocytes, microglial cells, and ODC progenitors. Myelin parts are engulfed by local microglia and macrophages, which are transformed into myelin-laden macrophages. Microglial cells are activated in response to tissue destruction. Activated macrophages and microglial cells act as APC and reactivate activated $\mathrm{CD}^{+}{ }^{+}$and $\mathrm{CD} 8^{+} \mathrm{T}$ cells to Th and CTL. Osteopontin, IL-23, and other cytokines, released by astrocytes, shift Th differentiation toward Th1 and Th17 phenotypes. Th and CTL release cytokines, other mediators of inflammation, and/or lytic products that elicit a full-blown inflammatory response which extends tissue damage. $\mathrm{NO}$, released from astrocytes and macrophages, alters the structure of myelin. (A) CNS antigens leak through soluble and cellular routes into lymph node. Alternatively, antigens could be collected by DC inside the CNS, and then DC travel into the lymph node. Antigens are presented to $\mathrm{CD}^{+}$and $\mathrm{CD} 8^{+}$cells by DC in the lymph node. Activated T cells enter the CNS. (B) Alternatively, microbial antigens are presented to $\mathrm{CD} 4^{+}$and $\mathrm{CD}^{+}$cells by DC in the lymph node. Activated T cells migrate into the CNS, where they are reactivated by macrophages and microglial cells in order to become fully differentiated effector cells. Redox processes involved in initial myelin degradation are important for the activation and reactivation of $\mathrm{T}$ cells (A) or for the reactivation of $\mathrm{T}$ cells (B). They are also involved in the modification of antigens, and in epitope spreading that is important for the perpetuation of autoimmunity. In response to activation, $\mathrm{T}$ cells differentiate into memory cells, which are essential for supporting autoimmunity. Thus, besides the initiation of autoreactivity against the CNS, redox changes also contribute to its shift into chronic autoimmunity. The plus $(+)$ and minus $(-)$ symbols represent positive and negative effects, respectively, on activity or demyelination. IL, interleukin; ROS, reactive oxygen species. 
initiating an inflammatory reaction, which may result in demyelination $(38,469)$. It is important to note that NO, which is excessively generated by iNOS in the initial lesion, may have anti-inflammatory effects at this point. NO is known to suppress the expression of MHC II in macrophages and endothelial cells (188), thus hampering the reactivation of T cells. Primary neuronal and/or oligodendroglial damage activates microglia, which, in turn, up-regulates MHC class I expression and releases proinflammatory mediators. Activated microglia is capable of cross-presenting antigens and of efficiently reactivating $\mathrm{CD}^{+} \mathrm{T}$ cells within the $\mathrm{CNS}(37,310)$. Moreover, proinflammatory mediators released by microglia compromise immunosuppressive activity of the CNS resident cells and stimulate ODC and neurons to increase the expression of MHC I (310). Consequently, activated CTL that are specific for CNS antigens both directly and indirectly target myelin, ODC, and neurons via lytic mechanisms (Fig. 9A). It should be noted that activated $\mathrm{CD}^{+} \mathrm{T}$ cells also produce various cytokines and other mediators which sustain neuroinflammation $(310,390)$.

An additional/alternative pathway of autoimmunity initiation, which also involves redox misbalance within the CNS, is plausible. We have elaborated that redox instability leads to ODC and myelin damage and BBB dysfunction. In addition, we have discussed the possibility of activation of CNS-reactive T cells by molecular mimicry or bystander activation, that is, by microorganisms. A combination of these two spatially distant processes might significantly increase the possibility for the initiation of autoimmunity within the CNS. In short, T cells are activated in an immune response to a microorganism. As a consequence of redox-induced changes in the CNS, their passage through BBB is facilitated, as well as their encounter with myelin and other CNS antigens. As a consequence of redox-induced damage of myelin and/or ODC, microglia and macrophages are stimulated and ready to reactivate $\mathrm{T}$ cells. Reactivated $\mathrm{T}$ cells perform effector functions that establish inflammation and perpetuate damage in the CNS (Fig. 9B).

In some cases, such as in aggressive Marburg's type of acute MS, the inflammation develops very fast (49). On the other hand, Lucchinetti et al. showed that, within an average of 27 days of potential MS symptom onset, at which point the biopsy samples were taken, around $50 \%$ of examined patients showed signs of cortex inflammation and/or demyelination, involving the activity of $\mathrm{CD}^{+} \mathrm{T}$ cells and myelin-laden macrophages. Fortunately, even the development of the autoimmune component of MS is not irreversible. At the mean follow-up of 3.5 years after the initial symptoms, around $20 \%$ of patients at first showing the signs of cortex demyelination/ inflammation were diagnosed for CIS and did not develop MS (284). Correale et al. observed the presence of newly formed inflammatory lesions in benign MS cases at $10+$ years' followup (94). This indicates that the inflammatory component may be persistent but non-aggressive in benign MS. The probability and the tempo by which the autoimmunity develops in MS are most likely related to genetic predisposition of the immune system to react with the released antigens (417).

\section{K. Redox regulation of $T$-cell migration and infiltration}

In order to enter the brain, $\mathrm{T}$ cells have to migrate, recognize the site of entrance, and infiltrate. It has been shown that activated $\mathrm{T}$ cells are first captured to the vessel and that they then crawl on to the vascular endothelia. T-cell-endothelium interactions are mediated by adhesion molecules, chemokines, and their receptors (131). It appears that the key integrin receptor-integrin interaction in MS is established between VCAM-1 and VLA-4. Monoclonal antibodies against VLA-4 stop the crawling and transmigration of myelinreactive $\mathrm{CD}^{+}{ }^{+} \mathrm{T}$ cells into the CNS parenchyma of model animals and restrict BBB transmigration of both non-effector and effector memory $\mathrm{CD}^{+} \mathrm{T}$ cells acquired from the CSF of MS patients $(34,215)$. The transmigration of T cells across $B B B$ also involves their interaction with pericytes, which is established through VCAM-1/VLA-4 pair (451). The importance of VCAM-1/VLA-4 interaction in MS pathogenesis is clearly delineated by the efficacy of anti-VLA-4 monoclonal antibody (natalizumab) in the treatment of MS patients (214). It has been recently demonstrated that this antibody also downregulates the production of soluble VCAM-1 in endothelial cells (319). The expression of VCAM-1 in endothelial cells is regulated by NF- $\kappa \mathrm{B}$ and promoted by oxidative stress (294). It should be emphasized that effector T cells transmigrate more readily across $\mathrm{BBB}$ than non-effector $\mathrm{T}$ cells, and that endothelium promotes the selective recruitment of effector $\mathrm{T}$ cells (215). Endothelial and brain cells may release soluble signals (chemokines) to "invite" T cells. After the initial engagement of chemokine receptors in $\mathrm{T}$ cells by chemokines, inside-out signaling pathways induce integrin activation in these cells (240), and, therefore, efficient interaction of integrins and their receptors is allowed. Chemokines may act either by creating a soluble chemotactic gradient or by being associated with extracellular matrix components or they may bind to cellsurface proteoglycans of endothelial and other cells (204). The most important chemokines in MS appear to be CCL2 (receptor-CCR2), CCL3, CCL4, CCL5 (share the common receptor-CCR5) (434), CCL20 (receptor-CCR6) (60), CXCL10 (receptor-CXCR3) (408), and CXCL12 (receptors-CXCR4 and CXCR7) (13) (Fig. 10A). All these chemokines are released from activated astrocytes $(13,55,304,434)$. Regulation of their expression involves NF- $\kappa \mathrm{B}(55,237,304,462,492)$. This implies that in the initial lesion, prooxidative processes and upregulated NF- $\kappa$ B expression in astrocytes and other cells may promote the infiltration of T cells (Fig. 10). Pertinent to this, the release of CXCL12 and CCL20 from astrocytes in MS lesions has been documented (13). Th17 cells show the most robust chemotaxis toward CCL20 (60). However, non-effector myelin-reactive $\mathrm{CD} 4{ }^{+} \mathrm{T}$ cells (activated in the lymph node; see Fig. 9), which may be present in CSF or blood, also express CCR6 and respond to CCL20 $(332,475)$. This may be crucial for the inflammation of the initial lesion, which further results in the development of a fully active autoimmune system. CCL3 is known to promote the chemotaxis and infiltration of activated CD8 ${ }^{+} \mathrm{T}$ cells into CNS $(420,430)$. It has been reported that CCR5 is involved in the modulation of CD8 ${ }^{+}$ T-cell activity, but that it is not essential for the entry into the CNS $(166,167)$. The affected expression of CCR5 in humans does not prevent MS development, although it appears to be related to a milder course of disease, while the severity of EAE is not reduced in CCR5-knockout animals (434). CXCR3 receptor is present in both $\mathrm{CD} 4^{+}$and $\mathrm{CD} 8^{+} \mathrm{T}$ cells. The presence of this receptor in T cells is higher in MS patients than in healthy subjects, and shows a significant decrease during remission in MS patients (436). CCL2 appears to promote cerebral infiltration of both $\mathrm{CD} 4^{+}$and $\mathrm{CD} 8^{+} \mathrm{T}$ cells (77). A 
A
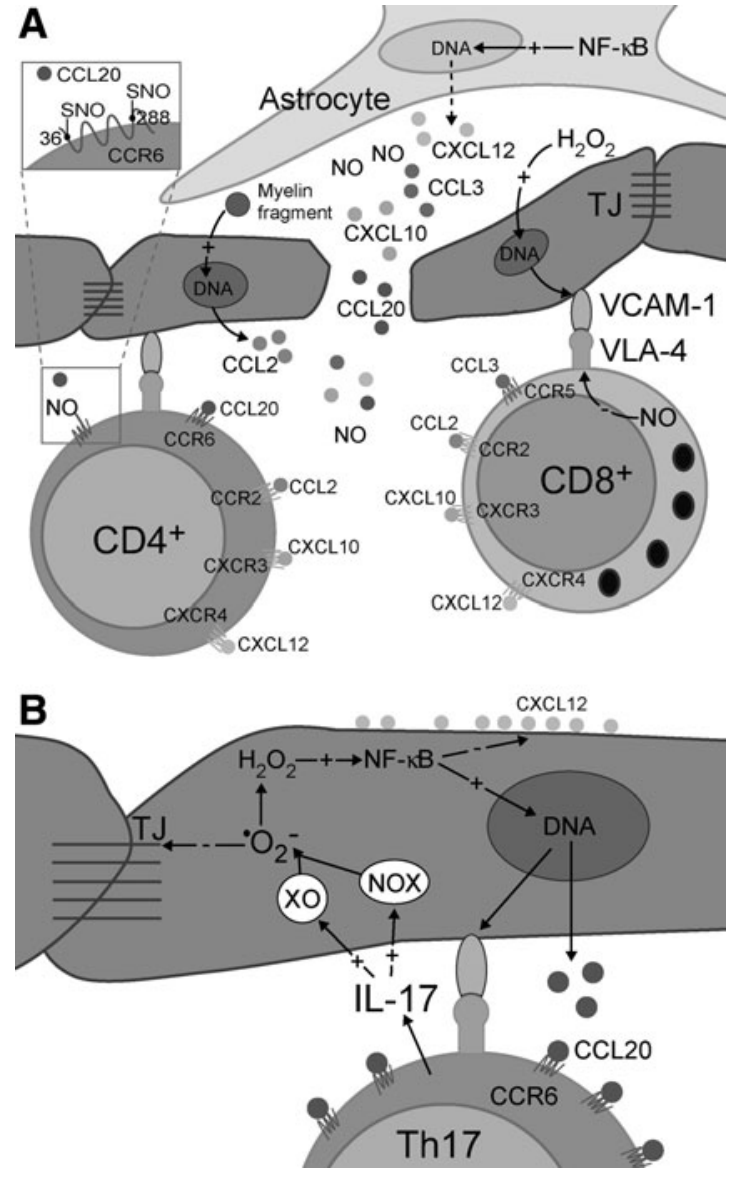

FIG. 10. Redox-regulated processes involved in the infiltration of $\mathrm{T}$ cells into the CNS. (A) The infiltration into the initial lesion. The activation of NF- $\kappa$ B in astrocytes promotes the release of a number of chemokines that bind to specific receptors on T cells, resulting in promoted infiltration. $\mathrm{H}_{2} \mathrm{O}_{2}$, released by astrocytes and macrophages, and myelin fragments promote the expression of VCAM-1 and CCL2 in endothelial cells. NO exerts anti-inflammatory effects by suppressing VLA-4 expression in T cells and by preventing chemokine binding. The latter is illustrated in an example of CCR6 in which Cys residues 36 and 288 may become S-nitrosylated. (B) The infiltration into the intact region of CNS during MS progression or in the case of cortical initiation of the disease. Th17 cells release IL-17, which promotes - $\mathrm{O}_{2}{ }^{-}$and $\mathrm{H}_{2} \mathrm{O}_{2}$ production in endothelial cells. These species affect TJ and activate NF- $\kappa \mathrm{B}$ cascade, which leads to increased production of CCL20 and decreased expression and redistribution of CXCL12 in the endothelial membrane, thus promoting the infiltration.

very recent study showed that the exposure of endothelial cells to MBP enables them to produce CCL2 (105), which may be related to their role as semi-professional APC. The severity of EAE is reduced in CCR2-knockout animals (434). In addition, it has been shown that MBP induces CXCL12 expression in astrocytes but not in endothelial cells (72). Altogether, this implies a very direct involvement of myelin fragments in the promotion of inflammation in (initial) lesions.

It has been reported that NO inhibits transendothelial migration of $\mathrm{T}$ cells $(414,474)$. This could be at least partially related to NO-provoked down-regulation of $\beta 1$-integrin
(VLA-4 subunit) expression in T cells (418). However, one additional mechanism is possible. It has been shown that some chemokine receptors include extracellular Cys residues with free thiol groups (e.g., $\mathrm{Cys}^{36}$ and $\mathrm{Cys}^{288}$ in CCR6), which are important for ligand binding. The mutation of these residues in CCR6 drastically suppresses chemotaxis of T cells (6). It has been proposed that thiol groups in chemokine receptors may be important for fine tuning the signaling properties of the receptors (374). They can be altered by S-nitrosylation or oxidation, the first process being probably more important for decreasing the infiltration (Fig. 10A), due to a larger diffusion radius of $\mathrm{NO}$ in comparison to $\mathrm{H}_{2} \mathrm{O}_{2}$. However, this remains to be further elucidated.

The infiltration can be drastically promoted when the Th17 phenotype is reached, as CCR6 is highly expressed by Th17 cells (475). In addition, Th17 cells also secrete the "prooxidative" cytokine IL-17 (213). IL-17 promotes ${ }^{\bullet} \mathrm{O}_{2}{ }^{-}$and consequent $\mathrm{H}_{2} \mathrm{O}_{2}$ production in endothelial cells, thus activating canonical NF- $\kappa \mathrm{B}$ signaling pathway (471). This stimulates the release of CCL20 and further infiltration of Th17 cells (60) (Fig. 10B). Even more, canonical NF- $\kappa \mathrm{B}$ signaling inhibits basal CXCL12 expression in endothelial cells (286). Under physiological conditions, CXCL12 expression is localized on the surface of endothelial cells facing CNS parenchyma. This specific spatial presentation appears to have a role in retaining T cells, which entered the perivascular space, from advancing toward the brain tissue (326). The decreased expression or redistribution of CXCL12 in endothelial cells results in a more severe EAE $(299,317)$. It is also known that CXCL12 shifts Th1 cells toward Treg cells (309), and that it induces apoptosis in activated T cells (298). Importantly, we have discovered that NO inhibits CXCL12 expression in astrocytes and endothelial cells (unpublished observation). The ability of Th17 cells to activate IL-17-provoked BBB disruption (213), to self-invite themselves by inducing CCL20 production in endothelial cells, or even to produce CCL20 by themselves (Fig. 10B) (60, 475) may be particularly important for the development of new inflammatory lesions in the previously unaffected brain regions during the course of MS. Furthermore, it has been shown in the EAE model, that as the disease progresses, Th17 infiltration becomes CCR6 independent (377), which may be related to a more general and severe loss of BBB integrity. It should be noted that epithelial cells of choroid plexus constitutively express CCL20, which may be one of the causes for the presence of $\mathrm{CCR}^{+}{ }^{+}$and $\mathrm{CD}^{+}$cells in the CSF of patients early in MS (377). Hence, IL-17-promoted CCL20 release may be particularly important for cortical infiltration (see the next section). Altogether, these facts may explain the notoriety of Th17 cells in MS pathology. It is worth mentioning that a subpopulation of effector memory $\mathrm{CD} 8{ }^{+} \mathrm{CD} 161^{+} \mathrm{T}$ cells which release IL-17 and express CCR6 receptors has recently been detected in the peripheral blood of MS patients (17).

\section{The outside-in initiation of MS}

Currently, there is a heavy debate within the MS research community regarding the chain of events that initiate clinically determined MS. Generally speaking, there are those who favor the "inside-out" hypothesis, that is, damage to the brain comes first followed by an autoimmune reaction (which has been outlined in detail in previous sections), and those who believe that CNS-specific autoreactive lymphocytes are the 
initial pathogenic entities ("outside-in"). The latter hypothesis is corroborated by EAE models, which involve the activation of $\mathrm{T}$ cells at the periphery, resulting in pathological processes within the CNS. EAE studies, especially those using transfer EAE models, clearly show that autoaggressive $\mathrm{T}$ cells infiltrate the CNS in order to induce MS-like pathology in experimental animals. Accordingly, the association of immune cells with MS lesions is evident. However, until recently, there have been no reports showing significantly increased presence of lymphocytes in early MS lesions. The key study came from Ransohoff's group, which performed histopathological examinations of cortex biopsies obtained in passing during diagnostic procedures targeting white matter lesions, within days or weeks after MS presentation (284). This is the first study adequately substantiating previous assertions about the development of cortical lesions early in MS $(107,111,363)$. Of the 138 patients, 38\% had cortical lesions showing signs of inflammation, demyelination, and neurodegeneration. Diffuse meningeal inflammation was significantly and strongly associated with cortical demyelination. The presence of $\mathrm{CD}^{+}$cells $\left(\mathrm{CD}^{+}\right.$is a specific immunohistochemical marker for $\mathrm{T}$ cells in general) and $\mathrm{CD}^{+}$ $\mathrm{T}$ cells has been documented in a majority of lesions, whereas the data on $\mathrm{CD}^{+} \mathrm{T}$ cells have not been obtained $(284,369)$. Reboldi et al. also found $\mathrm{CCR}^{+} \mathrm{T}$ cells in early cortical lesions, which implies that $\mathrm{T}$ cells infiltrate CSF using choroid plexus/CCL20 route (377) and may proceed to the cortex by provoking IL-17-dependent redistribution of CCL20 on the walls of blood vessels (103). It is important to note that meningeal aggregates of $\mathrm{T}$ cells are frequently formed around blood vessels in pia mater $(34,284)$. This could be explained by the fact that the cells coming from CSF find themselves on the CCL20-presenting side of the endothelium. Lucchinetti et al. proposed that macrophages carrying fragments of myelin may leave the cortex and spread epitopes to lymph nodes, which may result in T-cell activation $(284,369)$. Alternatively, $\mathrm{T}$ cells could be activated due to molecular mimicry, which seems to be a more probable cause (Fig. 11). It has been proposed that memory $\mathrm{T}$ cells may enter CSF in the subarachnoid space across the choroid plexus epithelium (244) or via transvascular route (34). The presence of $\mathrm{CD} 4^{+}$and CD8 ${ }^{+}$ memory $\mathrm{T}$ cells has been identified in the CSF of MS patients, non-inflammatory neurological disease patients, and even in healthy CSF $(130,222,245)$. Analogously, myelin-reactive memory $\mathrm{T}$ cells may also circulate in CSF. At some point, these cells may be reactivated by meningeal macrophages and become effector cells, with the interaction being mutually stimulatory (284). Pertinent to this, it has been shown, using the EAE model, that the first myelin-reactive $\mathrm{T}$ cells in the CNS appear before the onset of clinical EAE. The arriving cells are restricted to subarachnoidal space and remain strictly in close association with pia mater, crawling on the surface and on the outer face of blood vessels, performing a scan. There, T cells encounter APC meningeal/perivascular phagocytes, with the reactivation being followed by the escalation of the number of T cells (34).

At this point, redox changes may become crucial (Fig. 11). After activation, CTL and macrophages release ${ }^{\bullet} \mathrm{O}_{2}{ }^{-}, \mathrm{H}_{2} \mathrm{O}_{2}$, and $\mathrm{NO}$ (details presented in sections III.A. and B.). $\mathrm{H}_{2} \mathrm{O}_{2}$ and NO along with cytokines can pass through pia mater, a delicate membrane that separates the cortex from CSF. This may result in initial cortex damage and microglia activation, which has

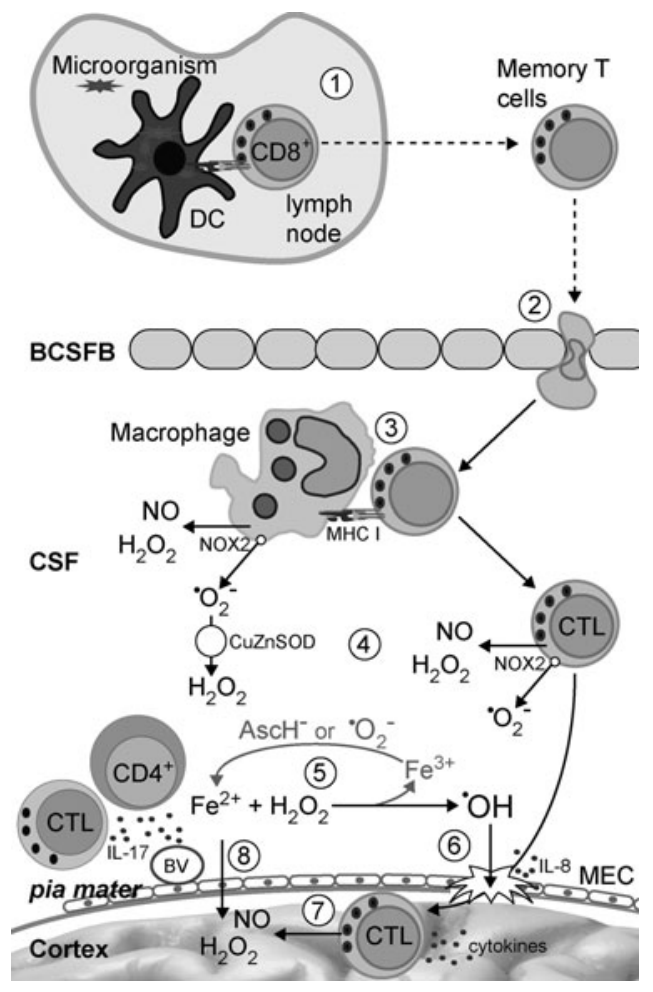

FIG. 11. The sequence of events in outside-in initiation of cortical lesions. 1 - $\mathrm{T}$ cells are activated via molecular mimicry, or via presentation of myelin fragments that are introduced into the lymph node by macrophages; 2 - Memory $\mathrm{T}$ cells pass BCSFB; 3 - The reactivation of $\mathrm{T}$ cells conducted by macrophages, resulting in mutual stimulation; 4- CTL and macrophages release ROS and RNS; $5-\mathrm{H}_{2} \mathrm{O}_{2}$ reacts with iron in the CSF. The reaction is promoted by Asc or ${ }^{\bullet} \mathrm{O}_{2}{ }^{-}$, which reduce the product form of iron - $\mathrm{Fe}^{3+}$ back to reactive form $\mathrm{Fe}^{2+}$; 6- Hydroxyl radical is produced, provoking oxidative damage to MEC and pia mater; 7- CTL invade cortex via damaged pia mater; and 8- alternatively, $\mathrm{H}_{2} \mathrm{O}_{2}$ and $\mathrm{NO}$ cross pia mater to provoke damage to the tissue, which leads to microglia activation, BBB breakage, and perivascular infiltration. IL-17 from $\mathrm{T}$ cells affects $\mathrm{BV}$ and provokes $\mathrm{BBB}$ disruption, or it may impact the integrity of pia mater. Asc, ascorbate; MEC, meningothelial cells.

been related to overlying meningeal infiltrates (287), followed by BBB breakdown, epitope spread, and perivascular T-cell infiltration (284). The involvement of T cells, which promptly emit high amounts of IL-17 on activation (103), may be particularly important. IL-17 has been reported to induce ${ }^{\bullet} \mathrm{O}_{2}{ }^{-}$ production in brain endothelial cells, resulting in $\mathrm{BBB}$ disruption (213). However, an alternative scenario is also plausable, taking into account that (i) both perivascular and submeningeal infiltration of $\mathrm{T}$ cells into the CNS of an EAE model have been observed (34); (ii) subpial cortical lesions are more frequent in early MS compared with the lesions formed around vessels (284); and (iii) CSF is significantly different fluid when compared with plasma, showing to be a more fertile ground for prooxidative processes. Namely, CSF shows lower levels of iron-binding proteins and several-folds higher concentrations of ascorbate (Asc) in comparison to plasma $(78,190,409)$. The reaction of iron with $\mathrm{H}_{2} \mathrm{O}_{2}$ (Fenton reaction) can be easily boosted during meningeal inflammation by 
- $\mathrm{O}_{2}{ }^{-}$(via Haber-Weiss reaction) or by Asc $(62,409)$, and some CSF metabolites also (see section IV.C.) (Fig. 11). This results in excessive production of the most reactive species in the human body- ${ }^{-} \mathrm{OH}$. This radical can damage pia mater, thus enabling the direct infiltration of CTL into the cortex, resulting in the fast development of inflammatory lesions. Pertinent to this, it has been documented that even mild oxidative stress drastically impairs the function and affects the viability of meningothelial cells (MEC), which line the surface of pia mater and are connected by TJ (472). It can be speculated that IL-17 may also affect TJ in this cell layer. It appears that MEC not only act as covering cells, but may also be involved in the inflammation, as, when exposed to oxidative stress, they release IL-6 and IL-8 (a chemotactic factor) (135). It should be noted that meningitis-provoking pathogen Streptococcus pneumoniae, which has been associated with the highest frequency of neuronal damage, employs $\mathrm{H}_{2} \mathrm{O}_{2}$ to damage meninges (202).

We propose that the early formation of cortical lesions involves different mechanisms compared with white matter lesions due to specific redox- and immune-relevant settings: (i) According to the presence of T cells in healthy CSF, BCSFB appears to be easier to cross than BBB; (ii) the cortex is the CSF-adjacent tissue, separated from the T-cell containing medium (CSF) only by one thin membrane (pia mater). This makes the cortex a site that may be more prone to the soluble mediators of inflammation (ROS, RNS, and cytokines) and to $\mathrm{T}$-cell infiltration comparing with white matter, which is shielded by a complex and thick BBB. (iii) In comparison to blood, CSF represents a more prooxidative environment, which may favor oxidative damage of pia mater under the inflammatory settings. It is tempting to further speculate what comes first, white matter lesions initiated by inside-out mechanisms or cortical lesions initiated according to the outside-in model. We believe that this varies from patient to patient, and that it may further reflect in different clinical courses of MS. For example, it has been previously shown that white matter lesions in PPMS show pattern IV $(283,366)$, which is mostly related to degenerative processes and may involve only a weak inflammatory component. However, a very recent study performed by Choi et al. has shown that diffuse meningeal inflammation and cortical lesions represent a hallmark of PPMS. The level of demyelination and neuron loss in the cortex was associated with the degree of meningeal inflammation (86). The progressiveness of symptoms in PPMS and the formation of cortical lesions appear to be closely related $(86,284)$. So in this case, white matter lesions may represent a reflection of global changes in the brain that are initiated by cortical lesions (369). However, white matter lesions represent the prevailing form of lesion in MS; so we believe that, in most cases, cortical lesions develop later, during progressive phases of MS (86), for example, during relapses in RRMS (66) or in SPMS $(254,358)$, which are characterized by meningeal inflammation (207).

\section{Redox Processes in Inflammation}

\section{A. Redox processes in macrophages}

In order to elaborate redox changes that are directly related to inflammation, we will focus on pattern II lesions. In pattern III lesions, the effects of prooxidative activity of the inflammatory component are overshadowed by the production of reactive species in astrocytes and other CNS cells, and, therefore, they would be hard to tell apart. It has been reported for pattern II lesions that the relative distribution of $\mathrm{T}$ cells and macrophages/microglia resembles the one seen for oxidative damage markers. In addition, the number of $\mathrm{T}$ cells and macrophages shows statistically significant correlation with the level of oxidation of DNA and lipids (181). However, pattern II lesions show approximately an 8-fold lower level of oxidation and 1-2 orders of magnitude lower PARP activity compared with pattern III lesions $(181,453)$. This implies that inflammation-unrelated processes involved in early/pattern III lesion development represent the main route of oxidative damage in MS. Inflammation appears to be significantly less responsible according to the comparatively modest redox changes in pattern II lesions.

In general, inflammatory stimuli are known to activate the expression of iNOS and COX2 in macrophages (29), and this has been observed in inflammatory, demyelinating (pattern II) lesions comprising catabolites of MBP and live ODC (384). The authors contemplated that prostanoids (COX2 products) and $\mathrm{ONOO}^{-}$may contribute to cellular death in the lesions. Nitrotyrosine depositions have been observed near, while the ingested nitrotyrosine positive material has been found inside the iNOS-positive inflammatory cells (384). iNOS and COX2 show a very interesting interplay (Fig. 12), which has been examined in macrophages and other cell types. $\mathrm{NO}$ is known to promote $\mathrm{COX} 2$ catalytic activity via $\mathrm{ONOO}^{-}$-mediated nitrosylation of specific $\mathrm{Cys}$ residue, but at high concentrations, NO inhibits COX2 activity and expression (30,112). On the other hand, prostaglandin PGD2 (COX2/isomerase product) can be metabolized to 15d-PGJ2, which is known to suppress $\mathrm{NO}$ production in microglial cells (120), while $\mathrm{H}_{2} \mathrm{O}_{2}$ that is produced by COX2/CuZnSOD activity may promote the expression of iNOS. These interconnected positive/negative feedbacks may act as switches in the regulation of inflammation. An important role of COX2 in the inflammatory component of MS is indirectly implied by a well-documented fact that cytosolic phospholipase A2-deficient mice are resistant to EAE (3). This enzyme produces arachidonic acid, which is the substrate of COX2. In addition, activated macrophages induce iNOS-dependent acyl chain nitration of a complex lipid cholesteryl linoleate. The product-nitrocholesterol linoleate $\left(\mathrm{CLNO}_{2}\right)$ suppresses iNOS expression and inflammatory responses in macrophages (30). Therefore, a key mediator of inflammation-NO-is involved in its eventual resolution. It should be noted that PGD2 and 15dPGJ2 have been shown to induce the apoptosis of ODC precursors (470); hence, the increased COX2 activity may result in hampered remyelination. Finally, excitotoxicity may be related to COX2 activity in macrophages, as prostanoids stimulate glutamate release (384). Another important enzyme that is expressed in macrophages in pattern II lesions is myeloperoxidase (MPO) (292). MPO generates hypochlorous acid $(\mathrm{HOCl})$, which is capable of provoking serious damage to biomolecules. However, due to its high reactivity, $\mathrm{HOCl}$ is considered as having a very limited diffusion radius in biological milieu. Therefore, only a small fraction (if any) of $\mathrm{HOCl}$ produced in macrophages may find its way out and affect the surrounding tissue (379).

Macrophages have to produce ${ }^{\bullet} \mathrm{O}_{2}{ }^{-}$and $\mathrm{H}_{2} \mathrm{O}_{2}$ in order to conduct the process of myelin phagocytosis. Inactive macrophages show a very low ROS production, which is boosted 


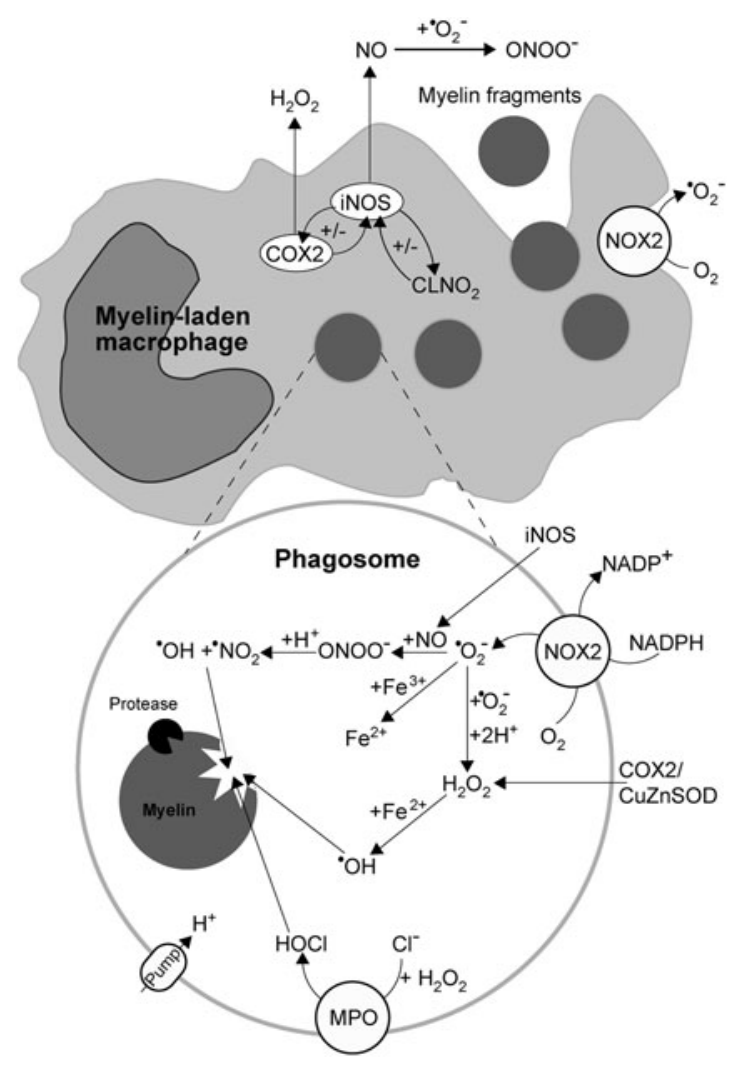

FIG. 12. Extra- and intracellular, and intraphagosomal redox activity and reactions in macrophages activated by myelin debris. In addition to the activity of proteases, the key players in myelin degradation are ${ }^{\bullet} \mathrm{OH}, \mathrm{HOCl}$, and ${ }^{\bullet} \mathrm{NO}_{2}$. The stimulation by myelin activates the expression of iNOS and COX2, the activity of which is mutually controlled by positive/ negative feedback effects and by $\mathrm{CLNO}_{2}$. NOX2 is present on both cellular and phagosomal membrane. Altogether, this results in a release of $\mathrm{NO}, \mathrm{H}_{2} \mathrm{O}_{2}$, and ${ }^{\bullet} \mathrm{O}_{2}{ }^{-}$from macrophages, and in extracellular $\mathrm{ONOO}^{-}$production. In phagosomes, ${ }^{\circ} \mathrm{O}_{2}{ }^{-}$ is dismutated to $\mathrm{H}_{2} \mathrm{O}_{2}$, which further reacts with iron to produce ${ }^{\bullet} \mathrm{OH}$, or reacts with $\mathrm{NO}$ to produce $\mathrm{ONOO}^{-}$, which is further decomposed to ${ }^{\circ} \mathrm{NO}_{2}$ and ${ }^{\bullet} \mathrm{OH}$. $\mathrm{Fe}^{3+}$ is reduced back to $\mathrm{Fe}^{2+}$ by ${ }^{\bullet} \mathrm{O}_{2}{ }^{-}$. MPO uses $\mathrm{H}_{2} \mathrm{O}_{2}$ and $\mathrm{Cl}^{-}$ions to produce $\mathrm{HOCl}$. Protonation $\left(+\mathrm{H}^{+}\right)$and Fenton reaction are promoted in phagosomes by low $\mathrm{pH}$, which is maintained by the activity of the $\mathrm{H}^{+}$pump. $\mathrm{CLNO}_{2}$, nitro-cholesterol linoleate; $\mathrm{HOCl}$, hypochlorous acid; MPO, myeloperoxidase.

approximately 10-fold, when myelin is present, but returns to the baseline level in the presence of NOX2 inhibitors (diphenyleneiodonium or apocynin). NOX2 is a multi-subunit enzyme that is dormant in resting phagocytes. After the activation by phagocytosis or some other stimulus, the complex is brought together on phagosome membrane, and it becomes active, delivering large quantities of ${ }^{\bullet} \mathrm{O}_{2}{ }^{-}$into phagosomal interior (395). In a set of experiments, van der Goes et al. incubated macrophages with myelin and specific ROS scavengers: catalase, CuZnSOD, and mannitol. The removal of $\mathrm{H}_{2} \mathrm{O}_{2}$ by catalase, and the scavenging of ${ }^{\bullet} \mathrm{OH}$ by mannitol suppressed macrophage-mediated myelin phagocytosis, whereas ${ }^{\bullet} \mathrm{O}_{2}{ }^{-}$removal by exogenous $\mathrm{CuZnSOD}$ did not show any such effects $(438,439)$. It is important to note that while mannitol can cross cellular membrane, the first two agents are relatively large proteins, which raise up the question: "How do these enzymes find themselves in a position to affect intracellular processes?" The only plausible answer is that in this experimental setup, catalase and CuZnSOD were taken up into phagosomes along with myelin fragments. The lack of effects of $\mathrm{CuZnSOD}$ implies that ${ }^{\bullet} \mathrm{O}_{2}{ }^{-}$is not a direct mediator of myelin degradation. Two superoxide radical anions can be dismutated to $\mathrm{O}_{2}$ and $\mathrm{O}_{2}{ }^{2-}$, with the latter being protonated to $\mathrm{H}_{2} \mathrm{O}_{2}$ (phagosomes show low $\mathrm{pH}$ values, which are maintained by the activity of $\mathrm{H}^{+}$pump). In addition, ${ }^{\bullet} \mathrm{O}_{2}{ }^{-}$reacts with $\mathrm{Fe}^{3+}$ from dead myelin/ODC to produce $\mathrm{Fe}^{2+}$ and $\mathrm{O}_{2}$. $\mathrm{H}_{2} \mathrm{O}_{2}$ is further involved in ${ }^{\circ} \mathrm{OH}$ production via a Fenton reaction (which is promoted by low $\mathrm{pH}$ ) and in MPO-mediated $\mathrm{HOCl}$ production. The main mediator of oxidative damage exerted on myelin fragments in phagosomes appears to be - $\mathrm{OH}$, as implied by the inhibitory effects of mannitol and catalase $(438,439)$. Superoxide may also react with $\mathrm{NO}$ that is generated by iNOS in the cytosol, to produce $\mathrm{ONOO}^{-}$and its derivatives- ${ }^{\bullet} \mathrm{OH}$ and ${ }^{\bullet} \mathrm{NO}_{2}$ (Fig. 12). The latter suppresses the inhibition of proteases and binds to proteins, which become more susceptible to protease activity. This could be very important for myelin degradation, as excessively oxidized proteins appear to be poor substrates for proteases (432). When it comes to the oxidative/nitrosative effects of macrophage activity on the surrounding cells, the main redox mediators are most likely $\mathrm{NO}$ and $\mathrm{H}_{2} \mathrm{O}_{2}$, both of which are produced in the cytosol by iNOS and COX2/CuZnSOD activity, because it is hard to believe that any significant amount of $\mathrm{H}_{2} \mathrm{O}_{2}$ could escape phagosomes. In addition to phagosome membrane, NOX2 is also present on the macrophage's cellular membrane (41) Therefore, NOX2-mediated ${ }^{\bullet} \mathrm{O}_{2}{ }^{-}$release into the extracellular compartment may also contribute to prooxidative effects of inflammation. Superoxide may be dismutated by CuZnSOD in the extracellular fluid (256), or it can react with $\mathrm{NO}$ in order to produce $\mathrm{ONOO}^{-}$. It should be noted that macrophages/microglia are highly resistant to damaging effects of $\mathrm{ONOO}^{-}(220)$, which implies that these cells use $\mathrm{ONOO}^{-}$for their function.

\section{B. Redox processes in T cells}

The activation and proliferation of $\mathrm{T}$ cells require a tightly regulated redox microenvironment, which $\mathrm{T}$ cells sense by their cell-surface thiol redox switches. The activation also involves ROS production by T cells, and the activity of APC (DC in particular) and Treg cells (477). The stimulation of TCR activates $\mathrm{CD}^{+}{ }^{+}$and $\mathrm{CD} 8{ }^{+} \mathrm{T}$ cells to express NOX2, dual oxidase 1 (Duox1) and eNOS (on cellular and outer mitochondrial membrane) $(221,255,336,422)$. The mechanisms behind this are not yet fully understood, but a few milestone papers provided a glimpse of some basic details. In the study performed on highly purified mature T cells, Williams' group observed three distinctive phases (pathways) of ROS production: (i) rapid (2-4 min after stimulation) and transient production of $\mathrm{H}_{2} \mathrm{O}_{2}$, independent of NOX2; (ii) NOX2dependent, sustained $\mathrm{H}_{2} \mathrm{O}_{2}$ production; and (iii) NOX2independent ${ }^{\bullet} \mathrm{O}_{2}{ }^{-}$production (221). In a subsequent study, the same research group found that the early $\mathrm{H}_{2} \mathrm{O}_{2}$ production (first phase) in stimulated $\mathrm{CD}^{+} \mathrm{T}$ cells is mediated by intracellular Duox1 (255). Yet another study on $\mathrm{CD}^{+}$cells showed that TCR stimulation leads to $\mathrm{H}_{2} \mathrm{O}_{2}$ production and calcium influx. The $\mathrm{H}_{2} \mathrm{O}_{2}$ production developed $2 \mathrm{~min}$ after 
stimulation, and was partially suppressed by rotenone, the inhibitor of ETC complex I (480). This implies that in addition to Duox1 activity, $\mathrm{H}_{2} \mathrm{O}_{2}$ leakage from mitochondria may account for the first component of ROS production in a stimulated $\mathrm{T}$ cell (221). The results showing that rotenone, a well-known promoter of ${ }^{\bullet} \mathrm{O}_{2}{ }^{-}$production on complex I (333), suppresses the emission of $\mathrm{H}_{2} \mathrm{O}_{2}$ from mitochondria are rather interesting and deserve further explanation. TCR stimulation-provoked calcium influx prompts mitochondria to work at a high capacity (336). In such a setting, mitochondria may lack ADP to continue ATP production, which results in increased proton gradient across the inner membrane and a high level of reduced co-enzyme Q. This leads to reverse electron transport from complex II toward complex I and to promoted ${ }^{\bullet} \mathrm{O}_{2}{ }^{-}$production (and increased $\mathrm{H}_{2} \mathrm{O}_{2}$ release), which is known to be abolished by rotenone (333). Calcium has also been reported to activate protease, which converts xanthine dehydrogenase to $\mathrm{XO}$, resulting in ${ }^{\bullet} \mathrm{O}_{2}{ }^{-}$production (413). This could potentially account for the third phase of ROS production in stimulated T cells, in addition to the prolonged promotive effects of calcium on respiration (336) (Fig. 13). It is worth mentioning that NO production in T-cell-APC conjugates is twice as high at cell-cell contacts than in the cytoplasm, and it represents a checkpoint in T-cell activation (336).

The intracellular production of $\mathrm{H}_{2} \mathrm{O}_{2}$ is needed for T-cell activation. For example, it has been reported that the radical scavenger $\mathrm{N}$-acetylcysteine (NAC) can inhibit T-cell activation (336). It should be noted that, on stimulation, GSH peroxidase-deficient $\mathrm{CD} 4^{+} \mathrm{T}$ cells proliferate at a faster rate compared with wild-type cells, while their differentiation is biased toward Th1 and Th17 cell development (466). Rotenone provokes a decrease in T-cell division and drastically suppresses the release of TNF and IFN- $\gamma$ from TCR stimulated (effector and memory) $\mathrm{CD}^{+}$cells (480). The application of a cell-permeable catalytic antioxidant (manganese (II) tetrakis (N-ethylpyridium-2-yl)porphyrin) on stimulated $\mathrm{CD}^{+} \mathrm{T}$ cells not only slows down the cell proliferation, but also suppresses the production of IFN- $\gamma$ and TNF $(405,480)$. Similar results have been observed for Duox1-knockdown $\mathrm{CD}^{+} \mathrm{T}$ cells (255). Therefore, the release of TNF and IFN- $\gamma$ from $\mathrm{T}$ cells appears to be promoted by $\mathrm{H}_{2} \mathrm{O}_{2}$, which is in line with the results obtained on some other cell types $(331,338)$.

On the other hand, the suppression of effector $\mathrm{T}$ cells and the induction of Treg cells depend on extracellular ROS production, mainly by NOX2 $(128,250)$. Pertinent to this, stimulated $\mathrm{CD}^{+}{ }^{+}$and $\mathrm{CD} 8^{+} \mathrm{T}$ cells are known to generate extracellular ${ }^{\bullet} \mathrm{O}_{2}{ }^{-}$by cellular membrane-bound NOX2, most likely for the purposes of self-regulation (422). Gelderman et al. showed that NOX2 activity increases the threshold of reactivity and suppresses proliferation of $\mathrm{T}$ cells by provoking oxidation of specific thiol groups $(-\mathrm{SH})$ on T-cell membranes (158). GSH treatment lowered the threshold of T-cell reactivity and promoted the proliferation of T cells, implying that cell surface redox -SH switches play a central role in the regulation of T-cell activity. However, the treatment with oxidized GSH (GSSG), which leads to decreased number of reduced thiol groups on the membrane surface by forming -S-SG moieties, results in T-cell suppression (158). The reaction of thiol group with $\mathrm{H}_{2} \mathrm{O}_{2}$ results in the formation of sulfenic acid $(-\mathrm{SOH})$ residue (104). GSH or Cys may reverse -SOH back to -SH. In two elegant studies, Yan et al. showed that after the contact with T cells or antigens, DC promote the activation and pro-

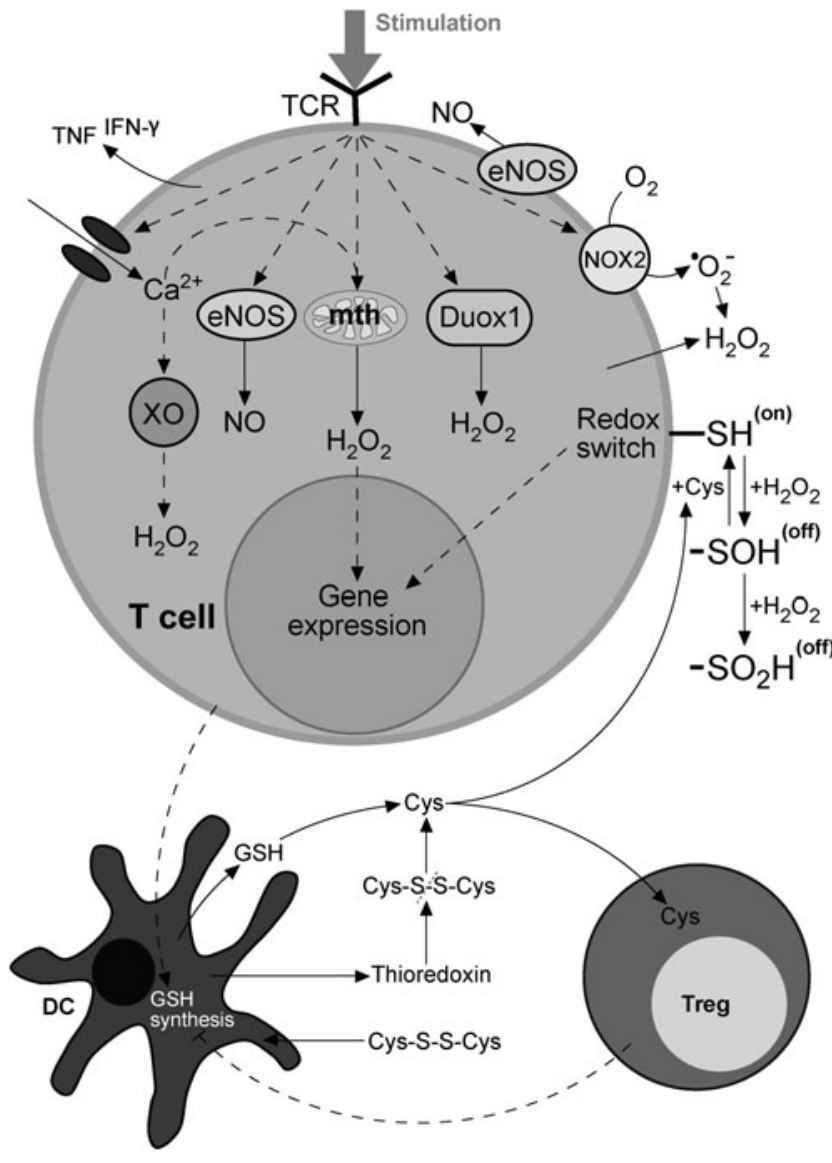

FIG. 13. Redox activity and regulation of TCR-stimulated $\mathrm{T}$ cell. In the intracellular compartment, the stimulation activates $\mathrm{H}_{2} \mathrm{O}_{2}$ production by mitochondria and Duox1. It also provokes calcium influx, which further promotes ROS production in mitochondria, and also by XO. eNOS is expressed in mitochondrial and cellular membrane (particularly in the vicinity of TCR). Cell surface -SH redox switch is "on" when reduced, and "off" when oxidized by $\mathrm{H}_{2} \mathrm{O}_{2}$. The first step of oxidation $(-\mathrm{SOH})$ is reversed to $-\mathrm{SH}$ by Cys. The excessive oxidation to $-\mathrm{SO}_{2} \mathrm{H}$ or $-\mathrm{SO}_{3} \mathrm{H}$ results in "irreversible off." Intracellular $\mathrm{H}_{2} \mathrm{O}_{2}$ and redox switch affect gene expression. Stimulated T cells promote GSH synthesis and oxidized Cys uptake in DC. DC recycle oxidized Cys and release GSH. Treg remove Cys from the extracellular compartment and suppress GSH synthesis in DC. DC release Cys and thioredoxin, which reduces extracellular oxidized Cys to Cys. Prooxidative intracellular and proreductive extracellular conditions promote T-cell activity and proliferation. DC are stimulated by $\mathrm{T}$ cells and create a reducing environment. Treg "fight" against this by inhibiting GSH production in DC and by removing extracellular Cys. The balance between DC and Treg activity determines the fate of T cells. Full line arrow, release or uptake of the product; dashed line arrows, indirect effects. Cys, cysteine; Duox1, dual oxidase 1; eNOS, endothelial NO synthase; GSH, glutathione.

liferation of T cells by releasing GSH, which is then cleaved to Cys $(478,479)$. Extracellular Cys increases the level of reduced thiol groups on the exofacial membrane proteins of $\mathrm{T}$ cells, thus turning the redox switch on (Fig. 13). In a co-culture with effector T cells, DC show enhanced GSH synthesis and the uptake of oxidized Cys (cystine; Cys-S-S-Cys). DC and T cells even release thioredoxin, which could recycle extracellular 
cystine to Cys $(16,247)$. On the other hand, Treg cells suppress DC-promoted inflammation by diminishing GSH synthesis in DC (by suppressing the expression of $\gamma$-glutamylcysteine synthetase), and by consuming extracellular Cys. It is important to note that the exposure to $\mathrm{H}_{2} \mathrm{O}_{2}$ excess may result in the apoptosis of $\mathrm{T}$ cells (419), which may be related to irreversible oxidation of $-\mathrm{SH}$ switches to $-\mathrm{SO}_{2} \mathrm{H}$ and $-\mathrm{SO}_{3} \mathrm{H}$. T-cell redox switches may also respond to other stimuli, such as heavy metals and carbonyl compounds that bind to thiols (8). Finally, it has been shown that myelin-laden macrophages inhibit TCR-triggered proliferation of myelin-reactive $\mathrm{T}$ cells in an antigen-independent manner by iNOS-mediated NO production (51). This implies that S-nitrosylation of thiol switches could be an important mechanism for regulation of the activity of $\mathrm{T}$ cells. The nature of redox switch(es) on T cells remains to be further elucidated.

It is clear that ROS and ROS-generating enzymes are involved in a complex signaling network which shows both pro- and anti-inflammatory effects. The concentration of $\mathrm{H}_{2} \mathrm{O}_{2}$ appears to be the key factor that defines the outcome (110). Figure 14 shows hypothetical curve of the dependence of effector T-cell activity/proliferation on $\mathrm{H}_{2} \mathrm{O}_{2}$ concentration. At the lower end, redox switches are on, but intracellular processes have not been initiated. At rising $\mathrm{H}_{2} \mathrm{O}_{2}$ concentrations, intracellular signaling is activated, while redox switches remain on. In this medium phase, the activity/proliferation is at its highest. A further increase in $\mathrm{H}_{2} \mathrm{O}_{2}$ level first turns redox switches (irreversibly) off, thus suppressing activity, and finally provokes apoptosis.

\section{Cytokines and the intracellular oxidative stress}

One of the important characteristics of MS is a massive release of cytokines within the CNS (270). The production of cytokines may modulate redox settings and vice versa. Taking into account the number of cytokines and the complexity of their effects and interactions, which could be a candidate for more than one review paper, here we will only illustrate redox/cytokine dependence on the example of IFN- $\gamma$ and IL-17. These are the signature cytokines of the pathogenic CD4 ${ }^{+}$ populations in the CNS autoimmune response, that is, Th1 and Th17 cells, respectively. Th1 proliferation and differentiation may be hampered by NO-promoted development of Treg cells (342), and by NOX2-related inhibition of IL-12 release from DC (224). However, experimental models of MS suggest that IFN- $\gamma$ plays an antioxidative role in neuroinflammation, as there is more oxidative stress in the CNS of
EAE-immunized IFN- $\gamma \mathrm{R}$ knockout mice than in controls (133), and as transgenic mice with suppressed ODC responsiveness to IFN- $\gamma$ develop EAE with an accelerated onset, associated with enhanced early inflammation and markedly increased ODC apoptosis. Accordingly, it was reported that IFN- $\gamma$ pretreatment of mature ODC in vitro has a protective effect against oxidative stress (31). Moreover, IFN- $\gamma$ stimulates microglia to uptake glutamate and, therefore, protects neurons (399). The differentiation of Th17 cells may be promoted by ROS generation in mitochondria (494). IL-17 collaborates with TNF in the induction of oxidative stress-dependent death of ODC (354). IL-17 induces BBB disruption through the induction of NOX- or XO-dependent ROS production (213). Thus, it appears that IL-17 has a positive relationship, while IFN- $\gamma$ has a negative relationship with ROS and oxidative stress in neuroinflammation. It is tempting to speculate that the different effects of IFN- $\gamma$ and IL-17 on oxidative conditions might be, at least partially, responsible for the observed variability in histopathological findings in EAE induced by Th1 and Th17 cells $(223,253)$.

\section{$D$. The contribution of oxidative stress to relapses and progression}

Relapses in MS may be related to newly emerging redoxinitiated processes in the CNS regions that are distant from the initial lesion (in the case of pattern III and IV lesions), or to significant changes in the immune system (in pattern I and II lesions); for example, to the occurrence of immune-compromised CNS and autoreactive memory $\mathrm{T}$ cells. Activated T cells differentiate into effector and memory $\mathrm{T}$ cells and later subdifferentiate into central memory $\mathrm{T}$ cells and effector memory T cells (387). Central memory T cells retain a high capacity for proliferation, while effector memory $\mathrm{T}$ cells retain a high effector capacity in response to antigenic challenge. Similarly, memory B cells subdifferentiate into classical memory B cells and plasma cells, which correspond to central and effector memory $\mathrm{T}$ cells, respectively (387). Therefore, rechallenging the immune system with an antigen will elicit a fast and strong response due to activity of memory $\mathrm{T}$ and $\mathrm{B}$ cells. It is fast, because cells are ready to perform effector functions, and strong, because there are antigen-specific cells that can intensively multiply in a short period of time. This concept is valid for MS pathogenesis, as activated memory T cells invade the CNS and induce novel neurological sequels or sustain those that have already been expressed. Indeed, the number of memory $\mathrm{CD}^{+}$and $\mathrm{CD} 8{ }^{+} \mathrm{T}$ cells is increased in the
FIG. 14. The hypothetical curve of the dependence of effector T-cell activity and proliferation on $\mathrm{H}_{2} \mathrm{O}_{2}$ concentration. Thiol switch(es) are "on" at low $\mathrm{H}_{2} \mathrm{O}_{2}$ levels, but intracellular redox signaling cascades are not activated. At increased $\mathrm{H}_{2} \mathrm{O}_{2}$ levels, redox switches remain "on," but redox signaling is now activated. A further increase in $\mathrm{H}_{2} \mathrm{O}_{2}$ concentrations results in the oxidation of $-\mathrm{SH}$ to $-\mathrm{SOH}$. In this form, redox switch(es) are (reversibly) "off." At even higher $\mathrm{H}_{2} \mathrm{O}_{2}$ levels, $-\mathrm{SOH}$ is further oxidized to $-\mathrm{SO}_{2} \mathrm{H}$ or $-\mathrm{SO}_{3} \mathrm{H}$. In this form, redox switch(es) are "irreversibly off."

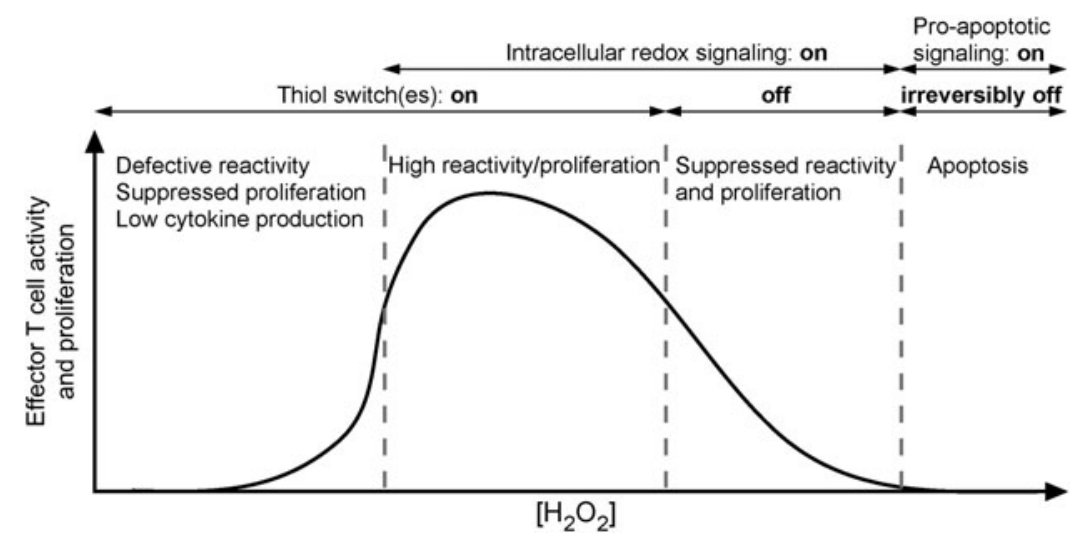


peripheral blood of MS patients, and these cells readily infiltrate CNS and are found in MS lesions $(25,180,243)$. Memory B cells are also implicated in MS pathogenesis, not only through the production of autoreactive antibodies, but also through antigen presentation and cytokine generation, which are highly relevant for T-cell functions $(219,308)$.

Although CNS-reactive memory cells are present in the immune system of MS patients, there should be a trigger that activates them and releases new attacks against the CNS. Here again, as in the initial autoimmune response, antigens might be released from the CNS that is damaged by oxidative/nitrosative stress. Pertinent to this, it has been reported that plasma levels of oxidation markers are higher in MS compared with healthy subjects (348), and that there seems to be a link between MS exacerbation and lipid peroxidation (426). It is known that the level of lipid peroxidation products, including oxidized low-density lipoproteins (oxLDL), is increased in the plasma and CSF of MS patients. Several different proinflammatory effects of oxLDL were reported (138). For example, oxLDL and ROS stimulate the generation of osteopontin (297), which plays a major role in MS pathogenesis (416), shifting Th differentiation toward pathogenic Th1 and Th17 phenotypes (177). Further, oxLDL increases Treg sensitivity to Fas-mediated apoptosis (307), thus provoking facilitated activation of autoreactive $\mathrm{T}$ cells. In addition, ROS and RNS heavily impact the effects of vitamin $\mathrm{D}$ by blocking its receptor function (253). Vitamin D3 is important for the optimization of branching of Asn (N)-linked glycans. Reduced branching results in T-cell hyperactivity and provokes inflammatory demyelination and neurodegeneration $(176,323)$. These facts implicate that oxidative/nitrosative stress may result in less restringent activation of autoreactive cells and their hyperactivity in MS.

ROS and RNS production in MS patients may be promoted by bystander infections. Bacterial and viral infections have been implicated in the induction of relapses and the acceleration of progression, whereas a vaccination against communicable diseases is considered beneficial for patients (280). Microbial stimulation of APC function and oxidative stressimposed hyperactivity of effector T cells might combine and lead to the activation of autoreactive encephalitogenic $\mathrm{T}$ cells and, thus, to relapses and MS progression. It should be noted that redox changes in inflammation result in epitope spreading, which is appreciated as highly relevant for chronicity in autoimmunity (448). Therefore, even if the initial autoreactivity is regulated and annulated, novel antigens still perpetuate autoimmune process. Importantly, it is not only the completely new antigens that emerge in epitope spreading, but also the (oxidative/nitrosative) modifications of existing antigens (22), which gives autoimmunity in MS a dynamic profile.

\section{E. Vitagene network, heat shock proteins, and inflammation}

The interplay between redox settings in CNS and inflammation may be indirectly mediated, by factors released from neurons and other cells in response to oxidative stress. Under stressful conditions, cells activate a vitagene transcriptional network (vitagenes encode for heat shock proteins (Hsp) Hsp32 (also known as HO-1) and Hsp70, the thioredoxin and the sirtuin protein systems), and the expression of other Hsp and small Hsp (sHsp; such as Hsp27 and HspB5 [ $\alpha$ B crystal- line]). It has been well documented that this takes place in MS lesions $(20,335,445)$. Integrated mechanisms controlled by vitagenes, Hsp, and sHsp operate in the brain in helping neuronal survival by performing folding and repair of damaged proteins, preventing aggregate formation and prooxidative/cytotoxic effects of free heme, and conducting protein triage. It should be noted that the vitagene network in lymphocytes is up-regulated in MS (361), most likely in order to prevent self-inflicted oxidative damage. However, in addition to their functions in the intracellular milieu, Hsp may be released into the extracellular compartment either by passive release from necrotic cells or via active release, including secretion in the form of exosome (e.g., the secretion of sHsp most likely represents altruistic delivery of supporting and stabilizing factors from one cell to another) (67-71). Released Hsp may act as immunomodulators, and some of them most likely play a rather important role in MS. The most relevant appears to be Hsp70, whose role in MS is still controversial. In a recent review, Mansilla et al. proposed that Hsp70 overexpression in CNS of MS patients has neuroprotective function. However, if this fails, and large number of neurons and ODC undergo necrosis, a certain amount of Hsp70, sufficient to promote or exacerbate inflammation, could be released. Proinflammatory effects of Hsp70 are most likely related to its activity as danger signal (Hsp70 promotes APC maturation and innate immune response) and adjuvant (Hsp70 binds to peptides and damaged proteins and creates new epitopes, which could potentially initiate endogenous molecular mimicry) (291). Hsp27 appears to act in a similar fashion, as some authors attribute the increased levels of this sHsp in MS to its neuroprotective effects, whereas others outline that it may act as an autoantigen. It is important to note that the level of Hsp27 in MS patients is increased during the phase of disease progression (80). Furthermore, the induction of T-cell tolerance for Hsp60derived epitope has been reported to ameliorate EAE (45). The abnormal expression of Hsp90 in lymphocytes appears to be responsible for the unresponsiveness of some MS patients to glucocorticoid therapy, as Hsp90 prevents the translocation of glucocorticoid receptor into the nucleus (296). In MS patients, Hsp90 is expressed at the surface of ODC precursors, which seems to promote their depletion in lesions (87). Although much work still has to be done, the general principle of "good inside CNS cells, potentially bad outside" may turn out to be applicable to most Hsp that are involved in MS pathology.

It has been proposed that HspB5 represents a special case, but it appears to us that there are just sufficient amount of data on this specific sHsp for elaborating all important aspects of its activity. The local concentrations of HspB5 may determine the balance between protective innate responses and destructive adaptive responses. In developing MS lesions, HspB5 is selectively induced as a protective protein in ODC and astrocytes, from which it is released in order to trigger microglia activation. The former appears to have neuroprotective effects at first, most likely as activated microglia remove debris. Pertinent to this, animals that do not express HspB5 show worse EAE. However, activated microglial cells also release cytokines and act as APC, which may ultimately overwhelm the protective microglial activity (445). This implies a very important role of HspB5 in transcending degenerative events in MS initiation to inflammation. The previous assertions that HspB5 may act as an autoantigen have been recently discredited by the finding that HspB5 binds to 
antibodies due to its chaperone characteristics (385). It seems logical to propose that other sHsp, the release of which is controlled, may not act as autoantigens. However, fragments of sHsp may act as autoantigens. For example, it has been shown that MMP-9 may cleave HspB5 in order to produce a promiscuous T-cell epitope (412).

\section{Neurodegeneration in MS}

\section{A. "Slow burning" of demyelinated axons}

Neurodegeneration of demyelinated axons is a major cause of irreversible neurological disability in MS. Partially or completely demyelinated neurons find themselves unprepared for a hostile environment in the CNS of MS patients, which shows increased levels of ROS, RNS, and iron. With their own weak AOS and without antioxidative and metabolic support, which would have been provided by a thick myelin sheets and glial cells (200), demyelinated neurons are highly susceptible to externally exerted oxidative/nitrosative stress (378) and to metabolic stress in general. However, the main cause of degeneration seems to come from within the demyelinated axon, and it may be viewed as "the triumph of function over survival." It is important to note that unshielded axons may be remyelinated by maturing ODC progenitors before serious damage occurs. However, the remyelination capacity is diminished in chronic lesions, so the axons remain permanently demyelinated.

Healthy neurons are energetically efficient cells. This is based on the insulating properties of myelin sheets, which enable fast, saltatory conduction of action potentials from one node of Ranvier to another. On demyelination, action potentials are propagated in a "wave-like" fashion, which requires increased expression and redistribution of $\mathrm{Na}^{+}$channels along the entire axon (97). The restoration of conduction comes at the cost of greater $\mathrm{Na}^{+}$influx and increased activity of $\mathrm{Na}^{+} / \mathrm{K}^{+}$ATPase, which is used for pumping out $\mathrm{Na}^{+}$, and it represents the largest ATP consumer in CNS even under physiological conditions (355). This increases the demands for ATP $(288,464)$, which are met by increased number of mitochondria (Fig. 15). It has been reported that demyelinated axons show a 2-3-fold increase of mitochondrial density compared with normal tissue $(288,464)$. More mitochondria means more $\mathrm{H}_{2} \mathrm{O}_{2}$, as there is a constant outflow of $\mathrm{H}_{2} \mathrm{O}_{2}$ from mitochondria into the cytosol. It has been reported that demyelinated neurons show increased levels of complex I and IV. While the activity of complex IV increases correspondingly, the activity of complex I, the main site of ${ }^{\circ} \mathrm{O}_{2}{ }^{-}$production in mitochondria, is impaired (464). According to this, NO may not play a direct role here, as it inhibits both complexes (333). However, even at lower concentrations, NO may, in combination with promoted ${ }^{\bullet} \mathrm{O}_{2}{ }^{-}$generation, result in a significant production of $\mathrm{ONOO}^{-}$, which is known to inhibit complex I, but shows significantly less pronounced inhibitory effects on complex IV (334). As a result, mitochondria in demyelinated axons experience oxidative stress, as implied by MtHsp70 chaperone overexpression and oxidative damage of mitochondrial DNA (464). Due to mitochondrial dysfunction and probably because of the decreased $\mathrm{O}_{2}$ availability (428), demyelinated neurons turn to increased glycolysis turnover in order to meet the high demands for ATP. Increased levels of lactate (glycolysis metabolite) have been detected in active MS lesions using MR spectroscopy
(355). In addition, Regenold et al. showed that the CSF of MS patients contains increased levels of metabolites of glycolysis and polyol pathway (380). Since NADH is produced in both these metabolic pathways, their up-regulation results in increased NADH $/ \mathrm{NAD}^{+}$ratio and consequent fulmination of ${ }^{-} \mathrm{O}_{2}{ }^{-}$production on complex I. The importance of this particular sequence of events is strongly implicated by $\mathrm{NAD}^{+}$ metabolism and its pharmacological effects in MS (360). It has been reported that the increase in $\mathrm{NAD}^{+}$level in EAE animals shows neuroprotective effects (226), most likely by decreasing $\mathrm{NADH} / \mathrm{NAD}^{+}$ratio. Brain in MS appears to try to compensate for $\mathrm{NAD}^{+}$decrease by de novo $\mathrm{NAD}^{+}$synthesis, which leads to a decrease in the level of tryptophan (the precursor of niacin and $\mathrm{NAD}^{+}$) in CSF and serum (350). Pertinent to this, a small group of MS patients were treated with tryptophan and they were showing symptoms alleviation (360).

At the end, the increased number of mitochondria and promoted glycolysis are not enough to meet energy demands of chronically demyelinated axons in the long run. Without enough ATP to power $\mathrm{Na}^{+} / \mathrm{K}^{+}$ATPase, neurons suffer from $\mathrm{Na}^{+}$accumulation, which at higher concentrations leads to reverse action of the $\mathrm{Na}^{+} / \mathrm{Ca}^{2+}$ exchanger (97), $\mathrm{Ca}^{2+}$ import, and $\mathrm{Ca}^{2+}$-induced cell death (355). It should be noted that $\mathrm{Na}^{+}$channel blockers and the inhibitors of $\mathrm{Na}^{+} / \mathrm{Ca}^{2+}$ exchange may provide protection from degeneration (228). Another factor that may lead to $\mathrm{Ca}^{2+}$ overload is the increased level of excitotoxic amino acids - glutamate and aspartate-in the CSF of MS patients (171). Calcium overload is known to activate NOX2 in neurons (57), as well as ROS production in mitochondria (121). At the final stage, demyelinated axons in chronic lesions show decreased presence of $\mathrm{Na}^{+} / \mathrm{K}^{+}$-ATPase (Fig. 15), which may represent an attempt to slow down the ATP expenditure (482). However, hand in hand with increased number of $\mathrm{Na}^{+}$channels, this results in a persistent ion imbalance, water influx, axon swelling, and degeneration (482). One MRI study documented that $\mathrm{Na}^{+}$level is up to twice higher in MS lesions compared with healthy tissue (218). Craner et al. showed that demyelinated axons try to fight $\mathrm{Na}^{+}$ overload by choosing to express $\mathrm{Na}_{\mathrm{v}} 1.2$ channels, as the persistent $\mathrm{Na}^{+}$current produced by $\mathrm{Na}_{\mathrm{v}} 1.2$ is significantly smaller compared with the current through $\mathrm{Na}_{\mathrm{v}} 1.6$ channels, which represent the predominant isoform in nodes of Ranvier (97). In addition, a small $\mathrm{Na}^{+}$-channel blocking oligopeptide (600-800 Da) was identified in the CSF of MS patients (24), most likely representing an adaptive factor aimed at decreasing $\mathrm{Na}^{+}$inflow into demyelinated axons (Fig. 15).

\section{B. Neurodegeneration in acute lesions}

1. White matter. The traditional view was that the inflammation is the cause of degeneration. Although it has been challenged by a number of studies (145), this concept may not be wrong, but distinction should be made between the neurodegenerative processes and the role of inflammation in neurodegeneration, which take place in the white matter and in the cortex (Fig. 16). In the white matter, the majority of axons degenerate by "slow burning" in inactive chronic lesions with depleted remyelination capacity. Nevertheless, it is clear that inflammation may propagate neurodegeneration in white matter $(403,407)$. Some axons may not survive acute inflammatory assault, and they undergo degeneration immediately after or even before the demyelination due to the 


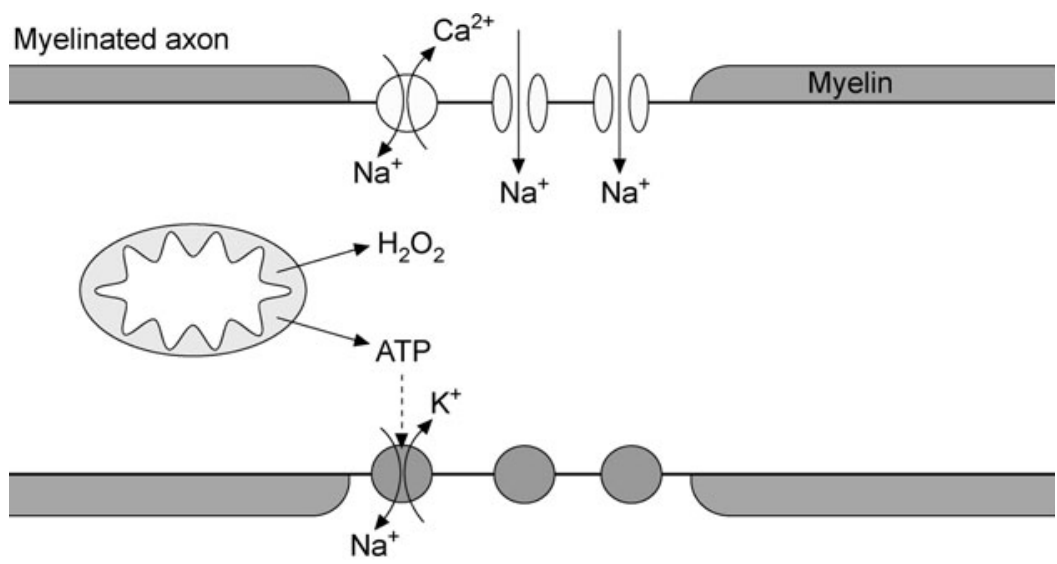

Demyelination $\quad \mathrm{Na}^{+}$-channel
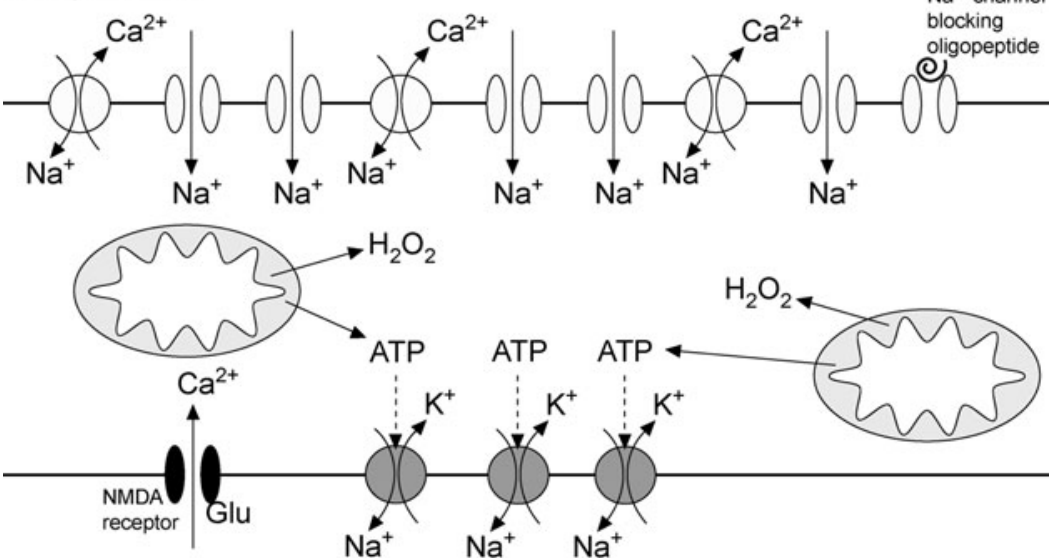

Neurodegeneration

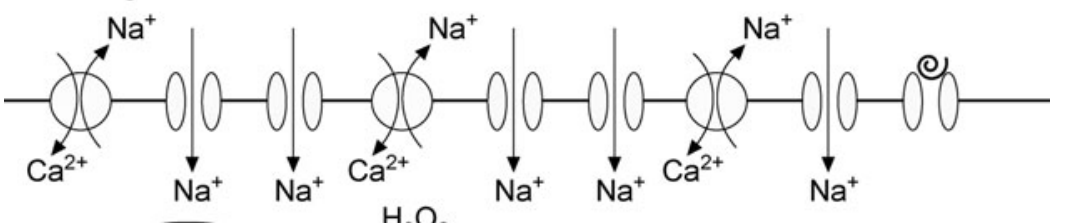
$\mathrm{H}_{2} \mathrm{O}_{2}$

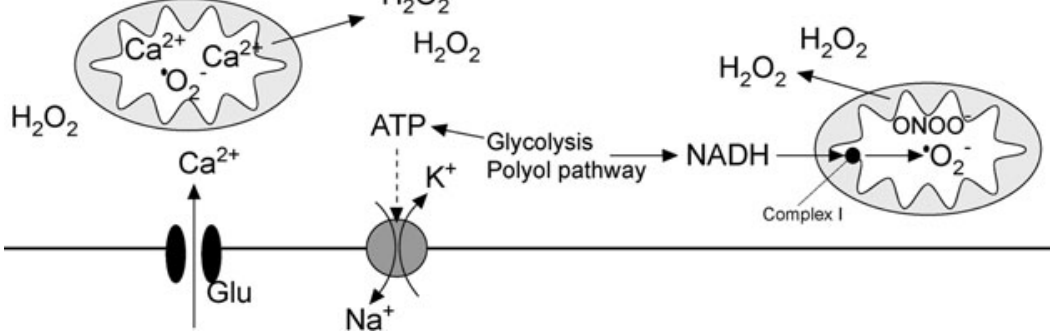

FIG. 15. Structural and redox changes taking place on the membrane and in the intracellular compartment of axons after demyelination and resulting in degeneration. Myelinated axons show normal number of $\mathrm{Na}^{+}$channels and $\mathrm{Na}^{+} / \mathrm{Ca}^{2+}$ exchangers, located at the nodes of Ranvier. The excess of $\mathrm{Na}^{+}$is pumped out by $\mathrm{Na}^{+} / \mathrm{K}^{+}$ATPase. In order to maintain their function, demyelinated axons show an increased number of $\mathrm{Na}^{+}$channels, which results in the increased activity of $\mathrm{Na}^{+} / \mathrm{K}^{+}$ATPase and ATP consumption. The cell tries to compensate the deficit of ATP by increasing the number of mitochondria and by activating glycolysis and polyol pathway. This results in increased $\mathrm{H}_{2} \mathrm{O}_{2}$ level, due to the increased baseline production in mitochondria as well as due to increased $\mathrm{NADH} / \mathrm{NAD}^{+}$ratio, which promotes ${ }^{\bullet} \mathrm{O}_{2}{ }^{-}$production in complex I. Still, increased ATP production is not sufficient for coping with $\mathrm{Na}^{+}$overload; so, the cell resorts to: the decreased presence of $\mathrm{Na}^{+} / \mathrm{K}^{+}$-ATPase, the release of small oligopeptide that blocks $\mathrm{Na}^{+}$channels, and the reverse action of the $\mathrm{Na}^{+} / \mathrm{Ca}^{2+}$ exchanger, which in combination with the effects of glutamate results in $\mathrm{Ca}^{2+}$ overload and $\mathrm{Ca}^{2+}$-induced cell death. impact of ROS, RNS, cytokines and glutamate released by T cells, activated microglia, and macrophages. Pertinent to this, accelerated deterioration of demyelinated neurons in active lesions in response to inflammation-generated NO has been documented (289). CTL have been reported to transect nonmyelinated axons in an MHC I-dependent manner (306). Finally, direct interactions between myelin-reactive Th17 cells and demyelinated axons resulting in damage have been observed in EAE (403). On the other hand, acute axonal injury has been shown to be partially independent of demyelination (48). In EAE model and MS biopsy samples, Nikić et al. observed a sporadically reversible process that they named "focal axonal degeneration," which is characterized by focal swellings that progress to axon fragmentation, in both demyelinated and myelinated axons. The process was related to the intra-axonal dysfunction of mitochondria that was provoked by macrophage-derived $\mathrm{H}_{2} \mathrm{O}_{2}$ and $\mathrm{NO}$ (343). In myelinated axons, the initial disruption typically took place at the nodes of Ranvier, which is in line with our concept that myelin represents a good barrier for ROS/RNS, while unprotected areas of axons represent weak spots.

2. Cortex. Cortical demyelinating lesions are widely present in MS patients, and may even represent the initial lesions in some cases. Importantly, cortical damage correlates with MS progression as well as with cognitive and other 


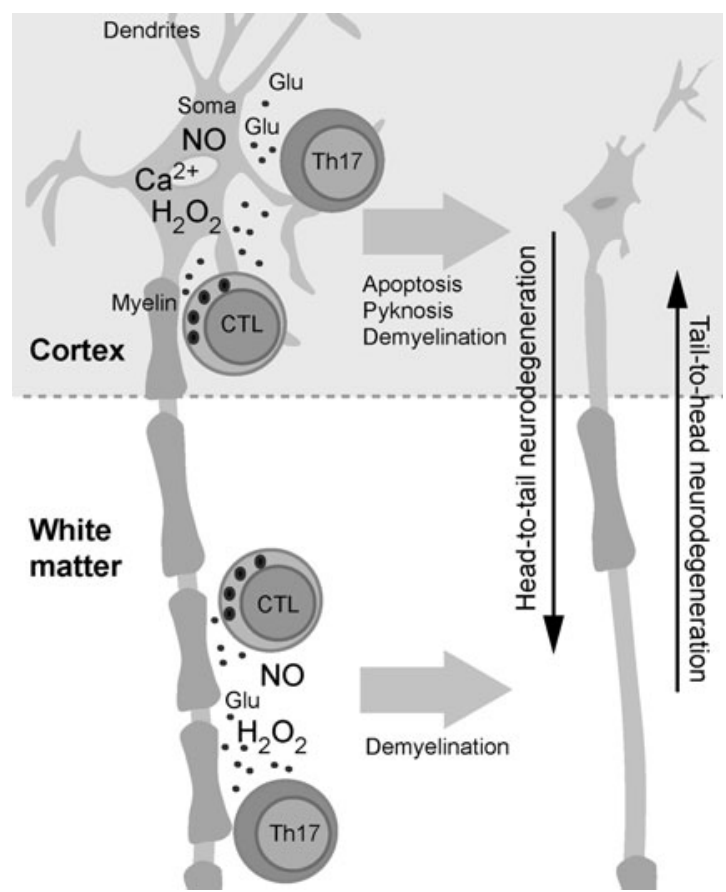

FIG. 16. The role of inflammation in neurodegeneration. In cortex, effector $\mathrm{T}$ cells attack myelin and affect neuron body via collateral bystander damage, or directly assault unprotected soma and dendrites, leading to cell death. $\mathrm{H}_{2} \mathrm{O}_{2}$ and NO may enter soma, which is also directly exposed to glutamate $(\mathrm{Glu})$ and cytokines. In white matter, myelin protects the axon; so, the major consequence of inflammatory attack is demyelination. These different events in cortex and white matter result in two different modes of neurodegeneration: head to tail and tail to head.

disabilities $(86,284,458)$. While the white matter is mainly composed of myelinated axons, cortex comprises both myelinated axons and unprotected neuron somas and dendrites. This difference may be reflected in the process of neurodegeneration, as neurons (neuron parts) that are unprotected by myelin are highly sensitive to RNS and ROS (152) and directly exposed to cytokines and glutamate. Neuronal injury, morphology changes, pyknosis and apoptosis, dendritic transection and the loss of dendritic arborization, as well as decreased density of neuronal somas and synapses are substantial in cortical lesions (284, 358, 363, 369, 370, 458). Papadopoulos and co-workers found a strong positive correlation between neuronal loss and inflammation in archaeocortex autopsies of MS patients, whereas axons appeared to be spared (358). In addition, phagocytic macrophages and lymphocytes are predominantly positioned close to somas and dendrites, while activated microglia do not ensheath the terminal ends of transected axons (axon "healing" may be futile if somas are affected) in inflammated cortical lesions $(363,369,403)$. Finally, working on an EAE model, Pomeroy et al. found the presence of cortical lesions and the loss of cortical thickness that is not related to demyelination (367). All this implies that an inflammatory assault on neuronal bodies takes place in active cortical lesions. T cells attack cortical myelin, as it is documented by the close relation between inflammation and demyelination in the cortex of MS patients (284). However, soluble mediators of inflammation may also affect nearby somas and dendrites [collateral bystander damage (407)], which are clearly more susceptible to damage compared with myelinated axons. Even more, dendrites can be cleaved directly by CTL that bind to MHC I on the neuron membrane and release soluble mediators (306). Giuliani et al. showed that polyclonally activated $\mathrm{CD} 4^{+}$and $\mathrm{CD} 8^{+} \mathrm{T}$ cells align along soma and (non-myelinated) axons of cultured human neurons and provoke neuronal death via antigen- and MHC-independent mechanisms. It is rather interesting that these effects were not reproduced by stable soluble products such as cytokines or when neurons and T cells were physically separated by a filter (165). This implies that the effects are based on direct cell-cell interactions and/or the involvement of short-living species with a shorter diffusion range compared with cytokines, that is, ROS and RNS. The importance of NO in cortical degeneration is implied by the decrease in the functional activities of ETC complexes I and III in mitochondria isolated from motor cortex of MS patients (126). There are some other circumstantial evidence with regard to the involvement of ROS and RNS in cortical neurodegeneration, such as increased expression of endoplasmatic reticulum stress-associated molecules in gray matter lesions (303), but this subject requires further investigation. $\mathrm{T}$ cells' binding to neurons most likely involves LFA-1/ICAM-1 interaction (165), and/or it is related to the fact that neurons express some myelin proteins (306). Zipp's group has shown that myelin-reactive Th17 cells provoke soma and axon degeneration by directly interacting with neurons and inducing glutamate-mediated excitotoxicity (403). Similar results were obtained in another study. It has been shown that activated $\mathrm{T}$ cells directly interact in an MHC-independent manner with soma and dendrites and provoke $\mathrm{Ca}^{2+}$ oscillations. This leads to a lethal increase in neuronal $\mathrm{Ca}^{2+}$ levels, which can be prevented by blocking glutamate receptors (344). It is important to note that in neurons, excitotoxicity and oxidative stress promote each other mutually (410).

The death of soma directly results in axon dysfunction and slow disintegration (we propose the term "head-to-tail" neurodegeneration), which is a vice versa process compared with retrograde degeneration starting from axons in white matter lesions and resulting in cell death at the end ("tail-tohead" neurodegeneration) (Fig. 16). According to this, the development of cortical lesions may have a more pronounced and straightforward impact on the patient's condition, as there are no mechanisms for damage reparation analogous to remyelination in white matter. It is important to note that axon-myelin or neuron-ODC interactions and metabolic support are bidirectional. For example, ODC need axons in order to efficiently switch to glycolysis in the case of ETC inhibition (e.g., by NO) (146). Therefore, the damage of neuronal body and axon may promote demyelination (431).

\section{Inappropriately chelated iron}

The mechanisms of neurodegeneration in MS, similar to neurodegenerative diseases, may involve oxidative pressure exerted by ${ }^{\bullet} \mathrm{OH}$ production in the Fenton system that takes place in the extracellular fluid and CSF, which are interconnected and poor in antioxidants (21). In MS, accumulated iron is present in the form of deposits in brain tissue $(27,383$, 487). The amount of iron in CNS positively correlates with the number of lesions, whereas the level of deposited iron shows a positive correlation with the neuro-psychological condition 

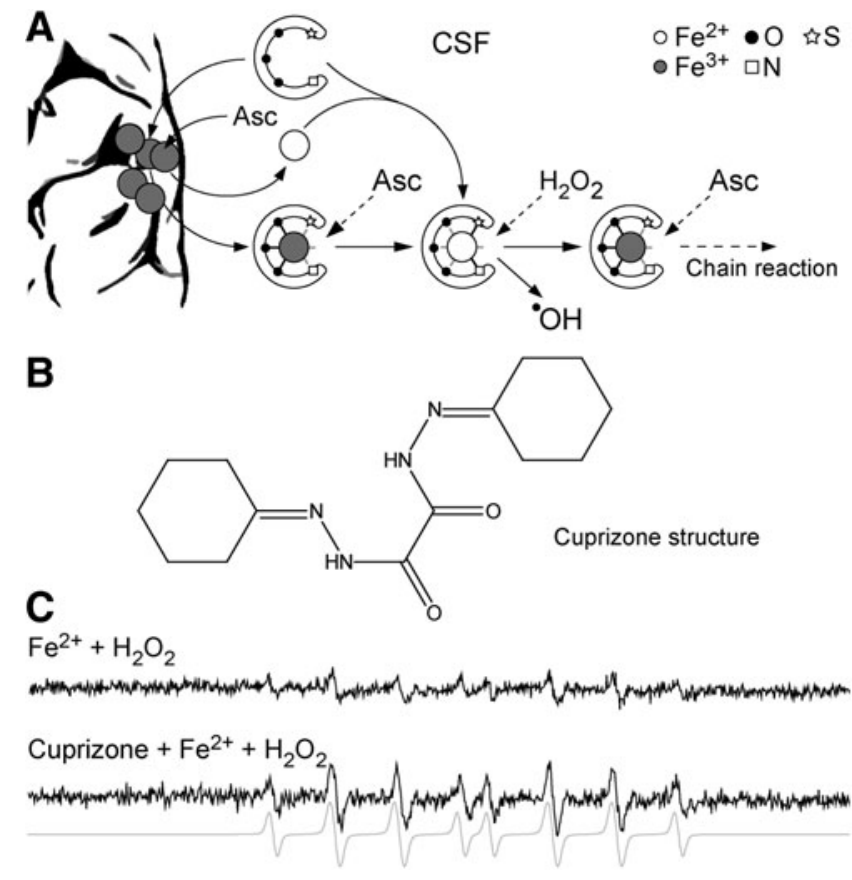

FIG. 17. Inappropriately chelated iron in MS and cuprizone model of MS. (A) The mechanisms of prooxidative activity of inappropriately chelated iron complexes in CSF of MS patients. Ligands or Asc may activate the iron from the deposits in the brain of MS patients. Inappropriately chelated iron is further involved in a redox chain reaction in which iron is alternately oxidized by $\mathrm{H}_{2} \mathrm{O}_{2}$ to produce ${ }^{\bullet} \mathrm{OH}$ radical, and reduced by Asc. The ligands that favor $\mathrm{Fe}^{3+}$ involve oxygen in iron binding, whereas ligands that bind $\mathrm{Fe}^{2+}$ involve nitrogen and sulfur. Therefore, biomolecules that are capable of binding iron in both redox states most likely comprise $\mathrm{O}$ and $\mathrm{N}$ and/or $\mathrm{S}$ in their structure in a configuration which allows the formation of two or three coordinate bonds. (B) The structure of cuprizone. (C) EPR spectra of spin adduct of ${ }^{\bullet} \mathrm{OH}$ radical (spin-trap DEPMPO, $15 \mathrm{mM}$ ) in human CSF supplemented with $\mathrm{FeSO}_{4}(0.2 \mathrm{mM})$ and $\mathrm{H}_{2} \mathrm{O}_{2}(1 \mathrm{mM})$ with or without cuprizone $(0.4 \mathrm{~m} M$ final concentration). The samples represent the leftovers from our previous study, and they were collected in accordance with the established international ethical guidelines (410). Gray - spectral simulation of DEPMPO/OH adduct. DEPMPO, 5-(diethoxyphosphoryl)-5methyl-1-pyrroline-N-oxide; EPR, electron paramagnetic resonance.

of MS patients (154), implying that iron may play a role in axon degeneration. In contrast, CSF levels of the two most important mediators of the Fenton reaction-iron and copper-are not increased in MS (313). However, the prooxidative activity of iron depends more on chelation and solubility than on the total concentration. This fact has recently been brought to attention by the concept of inappropriately chelated iron $(233,410)$. In brief, iron's prooxidative activity in the physiological milieu is limited by low $\mathrm{Fe}^{3+}$ solubility (it readily forms insoluble complexes with hydroxyl and phosphate ions), and is buffered by a number of chelating proteins. However, specific ligands may catalyze redox cycling of iron by increasing iron's availability to $\mathrm{H}_{2} \mathrm{O}_{2}$ and reducing agents, in particular Asc, which is accumulated in CNS reaching concentrations that are several-fold higher compared with plasma $(78,190)$. Iron and Asc represent a well-known "combination to avoid," as Asc promotes Fenton reaction (62, 410). Typically, iron can coordinate six ligands in an octahedral arrangement; so, molecules that are capable of binding to all six sites (e.g., transferrin) completely inactivate iron. By contrast, bidentate or tridentate chelators, which bind only to two or three chelation sites, increase the solubility and redox activity of iron (233). Generally, ligands that favor $\mathrm{Fe}^{3+}$ involve oxygen in iron binding, whereas ligands that bind $\mathrm{Fe}^{2+}$ involve nitrogen and sulfur (118). Therefore, biomolecules that are capable of binding iron in both redox states most likely comprise $\mathrm{O}$ and $\mathrm{N}$ and/or $\mathrm{S}$ in their structure in a configuration that allows the formation of two or three coordinate bonds. Such ligands can be rather dangerous, as they promote an ${ }^{\bullet} \mathrm{OH}$-generating chain Fenton reaction (Fig. 17A). CSF metabolome is significantly altered in MS compared with physiological settings, and there could be a number of potential iron accomplices in neurodegeneration, showing increased levels in the CSF of MS patients and meeting structural criteria. It is worth mentioning that we found increased prooxidative activity of inappropriately chelated iron in the CSF of patients suffering from a neurodegenerative condition (217).

Cuprizone is a copper-chelating agent which is applied in order to provoke MS-similar symptoms in animal models that are highly relevant for the investigation of pattern III and IV lesions (241). The mechanisms of cuprizone-activated demyelination are not fully understood, but they appear to involve metabolic stress in ODC and the activation of CXCR2 ${ }^{+}$neutrophils (276). Although it has been speculated that cuprizone promotes iron-related oxidative stress in the CNS (282), it has not been suggested to date that it may act by creating inappropriately chelated iron complexes. The structure of cuprizone (Fig. 17B) seems to allow the formation of such complexes. In addition, it has been proposed that cuprizone promotes $\mathrm{Cu}^{2+} / \mathrm{Cu}^{3+}$ redox cycling (179), which further substantiates our hypothesis. We performed a brief study using the CSF of patients with neurological conditions that are unrelated to iron metabolism (five lumbar and cervical disc herniations). Using electron paramagnetic resonance (EPR) spin-trapping spectroscopy, the production of ${ }^{\bullet} \mathrm{OH}$ radical in the CSF supplemented with $\mathrm{Fe}^{2+}$ and $\mathrm{H}_{2} \mathrm{O}_{2}$ was compared with the CSF co-supplemented with cuprizone. In all samples, the presence of cuprizone resulted in two- threefold increase of ${ }^{\bullet} \mathrm{OH}$ production (Fig. 17C), implying that cuprizone may form an inappropriately chelated iron complex. Since cuprizone primarily targets ODC (241), it is plausible that similar mechanisms may take place inside these iron-rich cells, resulting in oxidative stress and cellular death. Finally, inappropriately chelated iron complexes may play a role other than provoking oxidative stress. For example, carbonyl-iron has been reported to promote not only oxidation (148), but also the immune response. Actually, it has been used as an adjuvant for induction of extremely harsh EAE (315). We speculate that some inappropriately chelated iron complexes may act as natural adjuvants (149).

\section{Redox Therapy in MS}

\section{A. General strategy}

The development of effective (antioxidative) therapy for MS patients is intricated by the heterogeneity of the pathophysiological mechanisms involved. The redox therapeutic approach could target (i) degenerative component (ODC 
dystrophy being the hallmark), which predominates in initial and pattern III and IV lesions; (ii) inflammatory/autoimmune component, which is crucial in pattern I and II lesions; and/or (iii) neurodegenerative component, which develops as a result of the first two and involves the collapse of chronically demyelinated neurons. The main problem is that antioxidative therapies targeting different processes in degenerative and neurodegenerative component in MS pathophysiology may be futile or even show negative effects when it comes to autoimmunity, as the activation and proliferation of $\mathrm{T}$ cells require a tightly regulated redox microenvironment. In other words, the activity of $\mathrm{T}$ cells could be promoted, if supplemented antioxidants provoke a certain level of reduction. We will see later that the most promising agents currently available are able to target both degenerative and inflammatory components. The most accurate way to determine which component predominates in each MS patient is to determine the pattern of lesions. The therapeutic relevance of patterns is best documented by the findings of Keegan et al., who showed that only patients with pattern II lesions exhibit a significant functional neurological improvement after antibody depleting therapy (therapeutic plasma exchange); while this is not the case with pattern III and I patients (232). Currently, the patterns can be identified by means of biopsy/immunocytochemistry $(283)$ or potentially MRI $(53,398)$. It is important to note that most authors concur that one specific pattern is consistently observed in the samples from the same patient $(232,283)$. Some MS presentations are indicative of a specific pattern, but no significant correlation has been established between patterns and clinical manifestations (366).

Redox therapy should not be uniformly applied, and it should be developed to target specific mechanisms (patterns). For example, while many antioxidants delivered (variable) promising results in EAE studies, some, such as flavonoids, proved to be detrimental $(76,452)$. In addition, there is no substantial proof that any of the standard nutritional antioxidants (vitamins $\mathrm{C}$ and $\mathrm{E}$, lipoic acid, and others) have any effects on MS development or its progression $(137,490)$. It is about time for the scientific community to embrace the fact that antioxidants are not all the same and that they are not panacea. They act via specific mechanisms that may or may not interfere with the processes which are responsible for a particular disease (15). Here, we will mostly focus on redoxactive compounds that have been involved in clinical research and showed substantial benefits.

\section{B. Fumarates}

Probably the most exciting news for MS patients in a while came from the 64th Annual Meeting of The American Academy of Neurology, where the results of CONFIRM trial phase III have been presented (209). In phase II, oral application of dimethyl fumarate in RRMS patients for 24 weeks resulted in a reduced number of new lesions by more than $50 \%$ and a decreased annual relapse rate by more than 30\% (229). The treatment was prolonged to two years in phase III (involving more than 1400 RRMS patients), showing a reduction in annual relapse rate by around $50 \%$, and a decrease in the number of new lesions by $\sim 70 \%$ (143). The rationale for these trials was antioxidative/neuroprotective and antiinflammatory effects of dimethyl fumarate observed in vitro and in animal models (229).
Oddly enough for compounds that deliver antioxidative effects, fumarates seem to base their activity on binding to thiols via a Michael-type addition (393). Experiments performed on astrocytes and neuronal cells showed that shortterm exposure to fumarates results in decreased intracellular GSH level and short-lived oxidative stress $(10,268,392)$. However, long-term exposure showed quite the opposite effects, resulting in increased GSH levels and activated AOS (10, 268). Dimethyl fumarate stabilizes Nrf2 and promotes Nrf2dependent transcriptional activity. The first metabolite, monomethyl fumarate modifies Nrf2 inhibitor-Kelch-like $\mathrm{ECH}$-associated protein 1 (KEAP1) at $\mathrm{Cys}^{151}$. As a result, fumarates protect neurons and astrocytes from oxidative stress by activating Nrf2-controlled set of enzymes: NAD(P)H:quinine oxidoreductase 1 and GSH-related enzymes (271). Another study on astrocytes exposed to inflammation showed that fumarates reduce nuclear levels of NF- $\kappa \mathrm{B}$ p65 subunit, prevent the loss of $\mathrm{I} \kappa \mathrm{B}-\alpha$ (NF- $\kappa \mathrm{B}$ inhibitor), and drastically suppress iNOS expression (268). It should be stressed that the translocation of p65 subunit (as well as p50) and the activity of $\mathrm{I} \kappa \mathrm{B}$ kinase can be inhibited by thiol-reactive compounds targeting specific Cys residues (73, 116, 356) (Fig. 18). With regard to anti-inflammatory effects of fumarates, it has been reported that dimethyl fumarate provokes T-cell apoptosis both in vitro and in vivo (in cuprizone model of MS) $(324,429)$. The underlying mechanism most likely involves the irreversible binding of fumarates to thiol redox switches on T-cell membranes (Fig. 18). The modification resembles the effects of excessive oxidation (478). Finally, Ghoreschi and co-authors showed that fumarates provoke the activation of type II DC, which inhibit Th1/Th17 cells differentiation via a pathway involving impaired production of IL-12 and IL-23 (162).

Another compound that forms adducts with thiols, and, as we believe, may be of interest for future MS trials, is pyruvate. It shows many similarities with fumarate, and it is available as a dietary supplement, while its more stabile derivative - ethyl pyruvate-is approved for human use (227). Pyruvate shows neuroprotective effects against oxidative damage (455). Ethyl pyruvate exerts anti-inflammatory effects via GSH depletion, activates Nrf2 in astrocytes, inhibits NF- $\kappa \mathrm{B}$-dependent transcription by targeting p65 subunit, and suppresses the expression of iNOS $(183,227,401)$. In addition, pyruvate attenuates the effects of $\mathrm{ONOO}^{-}$(227). Its imunomodulatory effects should be further investigated.

\section{Cannabinoids}

A number of clinical trials have tested the ability of different cannabinoids or cannabinoid-containing products to alleviate MS symptoms. The beneficial effects on MS-related pain and sleep disturbances are well substantiated (486). A large long-term follow-up study (CAMS) indicated that cannabinoids may slow the progression of disability, increase mobility, and decrease relapse rate (485). However, preliminary results of another large trial (CUPID), presented at the annual meeting of The Association of British Neurologists in 2012, imply that tetrahydrocannabinol does not significantly slow the progression of disability in the general MS population, but may show beneficial effects in patients at the lower end of the disability scale (212). In relation to this, we speculate that cannabinoids do not target the neurodegenerative component of MS, which is responsible for the promotion of 


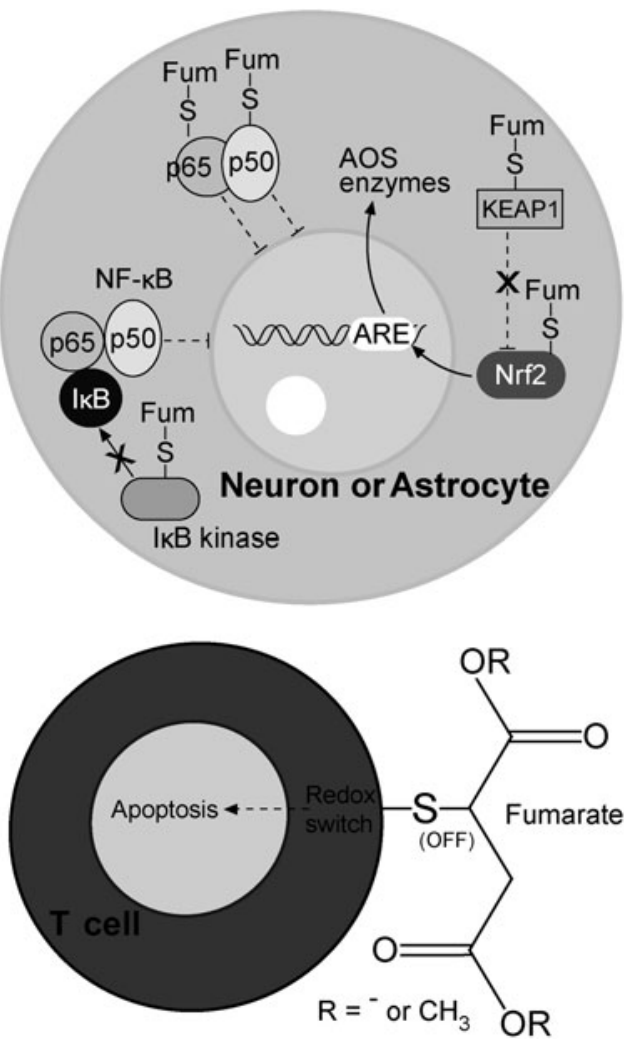

FIG. 18. The effects of fumarates on the activity of redoxrelevant transcription factors in neurons and astrocytes and on the redox switches in $T$ cells. The binding of fumarates on specific thiol residues prevents NF- $\kappa \mathrm{B}$ translocation to nucleus, $\mathrm{I} \kappa \mathrm{B}$ kinase-provoked release of $\mathrm{I} \kappa \mathrm{B}$, and the binding of KEAP1 to Nrf2, thus promoting its translocation into the nucleus and activating ARE expression. This may stop prooxidative cascade in astrocytes/neurons and promote antioxidative defense. Fumarates bind to T-cell-surface thiol redox switches, turning them off, which may result in apoptosis. The chemical structure of fumarates is presented. ARE, antioxidant response element; KEAP1, Kelch-like ECHassociated protein 1 .

neurological problems later during the MS progression, but may affect the degenerative and/or autoimmune component of MS pathophysiology.

The anti-inflammatory activity of cannabinoids involves the inhibition of proliferation and activity of effector T cells, the induction of Treg cells, and the inhibition of macrophage activity (388). In addition, cannabinoids are known to be potent antioxidants. In a comparative study, cannabidiol showed higher antioxidative capacity compared with butylated hydroxytoluene and vitamins E and C (182). Being highly lipophilic, cannabinoids most likely act as membrane-based antioxidants, and in MS, they could prevent HNE and MDA production. Some results imply that cannabinoids show ironchelating properties (235). It should be noted that a recent study showed that cannabidiol may ameliorate EAE in a direct, receptor-independent manner (249).

Two types of cannabinoid receptors (CBs) have been described: CB1 and CB2. CB1 is widely present in neurons, while CB2 is mainly found in immune cells (486), as well as in ODC precursors and astrocytes $(160,325)$. Neuroprotective effects of cannabinoids against oxidative stress are not mediated by CB1 receptors (293). On the other hand, the stimulation of CB2 receptors inhibits NF- $\kappa \mathrm{B}$ activation and iNOS and COX2 expression in astrocytes (265). In this way, cannabinoids may stop redox-cycle development in astrocytes in early or pattern III lesions. In addition, an in vivo study showed that cannabidiol suppresses neuroinflammation-provoked iNOS expression (134). Finally, cannabinoids affect ODC precursors via CB2 stimulation, promoting their survival and differentiation $(169,325)$. Cannabidiol is known to protect ODC progenitor cells from $\mathrm{H}_{2} \mathrm{O}_{2}$-mediated oxidative stress and inflammation-provoked apoptosis by decreasing the expression of endoplasmatic reticulum apoptotic effectors (305). It seems that future research on the application of cannabinoids in MS treatment should focus on cannabidiol, rather than tetrahydrocannabinol, given that the former showed more promising preclinical results; it is not psychoactive, and unlike tetrahydrocannabinol, its neuroprotective effects are not susceptible to tolerance development (193). In order to achieve the best results, cannabinoids should be applied early in MS.

\section{The inhibition of NF-KB and iNOS}

Some drugs that are approved for therapy of MS, as well as some of those which have shown beneficial effects in clinical or preclinical trials, are known to affect NF- $\kappa$ B-regulated expression and iNOS activity, which may represent an important component of their mechanisms of action. Mitoxantrone (DNA-reactive agent), which is used for the treatment of patients suffering from SPMS, PRMS, or RRMS worsening, is known to suppress NF- $\kappa$ B DNA-binding activity and NO production in stimulated astrocytes (64). Teriflunomide (the inhibitor of pyrimidine de novo synthesis), which is used for oral treatment of patients suffering from RRMS (211), inhibits iNOS in astrocytes (316). Methylprednisolone (synthetic glucocorticoid), which is widely used for acute treatment of MS relapses, inhibits NF- $\kappa$ B activation in inflamed CNS tissue and iNOS-mediated NO production in ODC $(205,473)$. Minocycline (tetracycline antibiotic) has been shown to decrease the development of new lesions and the relapse rate in MS patients $(312,484)$. This may be related not only to its immunomodulatory effects, such as suppressed antigen presentation and reduced T-cell activity $(246,386)$, but also to the ability of minocycline to inhibit iNOS and to down-regulate MMP-9 (484). The former effect is most likely mediated by NF$\kappa \mathrm{B}$ inhibition and may transpond to COX2 expression (65). Fluoxetine (antidepressant; trade names Prozac and Sarafem) has been shown to reduce the development of focal inflammatory lesions in MS patients (328). Importantly, it is effective in inhibiting microglia activation, including NF- $\kappa$ B-dependent generation of NO by iNOS (274). Natrexone (opioid receptor antagonist) improves mental health and decreases spasticity of MS patients $(99,164)$. It appears to act by preventing ODC death via the reduction of iNOS activity and $\mathrm{ONOO}^{-}$generation (5). Methylthioadenosine (naturally occurring sulfurcontaining nucleoside), which showed good results in preclinical studies, prevents the degradation of $\mathrm{I} \kappa \mathrm{B}-\alpha$, resulting in impaired NF- $\kappa$ B activation and suppressed iNOS activity (327). Epigallocatechin-3-gallate (green tea phenol) exerts protective and regenerative effects in neurons exposed to inflammation (195), which may be related to its ability to block the activity of 20S/26S proteasome complex, resulting 
in the accumulation of $\mathrm{I} \kappa \mathrm{B}-\alpha$, the suppression of NF- $\kappa \mathrm{B}$ activation (9), and probably inhibited iNOS expression (269). Mycophenolate mofetil (antimetabolite immunosuppressive prodrug), which, according to phase II trial, suspends MS progression and reduces relapse rate, is known to attenuate iNOS expression (281). Laquinimod (immunomodulatory agent) exhibited beneficial effects in the phase III trial on RRMS patients, slowing down the progression of disability and reducing relapse rate (89). The mechanisms of action may involve the protection of CNS cells from cytokine- or $\mathrm{H}_{2} \mathrm{O}_{2}$ provoked oxidative stress (311), and the suppression of NF- $\kappa$ B-regulated gene transcription (including iNOS) in astrocytes (61). Euphol (alcohol tetracyclic triterpene) not only attenuates the progression and severity of EAE, most likely by suppressing myelin-specific Th17 cells infiltration and cytokine production, but also attenuates NF- $\kappa$ B-regulated iNOS and COX2 expression in the CNS (125). Very similar effects in an EAE model were observed for metformin (antidiabetic drug) (339). Some studies showed that the application of selective iNOS inhibitor aminoguanidine leads to a significant decrease in the clinical expression of $\operatorname{EAE}(58,278,402,493)$, implying that iNOS inhibition may represent an important component of MS treatment. It appears that aminoguanidine may be worth trying in the treatment of early/pattern III MS. It is tempting to speculate that the inhibitors of NF- $\kappa \mathrm{B}$ and iNOS could provide the best results if applied early in MS.

\section{E. Other agents}

1. Iron chelators. Following several EAE studies, which delivered variable results, iron chelation strategy using deferoxamine was applied in a small trial on SPMS patients with no recognizable effects (411). However, the dose and the interval of application of deferoxamine were limited by a set of safety issues, and deferiprone could have been a more effective choice (321). Some interesting novel strategies have been proposed in iron chelation therapy, such as the development of chelators that are activated by oxidative stress (233). On the other hand, prooxidative activity of iron mainly relies on molecules that form inappropriately chelated iron complexes. Hence, the targeting of those molecules (which are yet to be identified) may represent the future of neurodegeneration treatment, including such processes in MS.

2. N-Acetylcysteine. NAC has been reported to attenuate oxidative stress in CNS of an EAE model (278). In addition, NAC attenuates HNE-provoked redox changes in endothelial cells and prevents pathological modification in TJ protein network (435). However, NAC appears to prevent T-cell apoptosis and activity suppression induced by different agents $(56,198)$, most likely by reducing the redox switches. According to this, NAC should be preferentially applied in pattern III patients. One phase II trial on RRMS patients has been conducted, but we are still waiting for the results to be published (208).

3. Ketogenic diet. A very recent EAE study showed that ketogenic diet slows down the development of motor disability and the loss of spatial learning and memory. The suggested mechanisms behind these effects involve amelioration of immune response and oxidative stress $(238,340)$. Ketogenic diet is known to inhibit ${ }^{\bullet} \mathrm{O}_{2}{ }^{-}$production on mito- chondrial complex I by promoting NADH oxidation, which results in decreased NADH/NAD ${ }^{+}$ratio (285).

4. Vitagene network activators. The results of several studies have shown that carnitine supplementation may improve the quality of life and reduce the symptoms of fatigue in MS patients (421), and a trial (FACTSEP) is underway that examines this issue (210). The positive effects of L-carnitine/ acetyl-L-carnitine may at least partially be attributed to their capacity to activate neuroprotective vitagene network (6771). A similar explanation may also be valid for recently published results, showing that natural polyphenols protect neurons in the chronic mouse model of MS and EAE animals (142). Finally, the activation of sirtuin protein system, which is encoded by vitagenes, has been shown to prevent the loss of neurons and axonal density and improve neuronal function in EAE (489). In relation to proinflammatory properties of Hsp70, it seems that the activators of vitagene network should be applied in the remittance phase in order to target neurodegeneration in chronic lesions.

\section{Concluding Remarks}

MS is a heterogeneous disease. A number of different components-vascular, redox, inflammatory/autoimmune and neurodegenerative, and various pathological patterns and phenotypes, combined with an astonishing amount of intricate data-may create an impression that this is an insolvable problem and an intractable disease. It is our belief that the extensive research should be accompanied by a comprehensive/integrative approach, in order to reach the true understanding of what happens in the body/CNS of an MS patient. The current review represents our humble attempt to do so. We have focused on redox processes, carefully trying not to over/underestimate their role in each step of MS pathogenesis, from initiation to neurodegeneration, and paying appropriate attention to other components, the autoimmune in particular. We have noticed some very important issues that should be addressed in greater detail in the near future: (i) the redox regulation of immune cells, especially $\mathrm{T}$ cells and APC; (ii) the involvement of redox processes in the regulation of chemokine network, and the differences between activated and reactivated $\mathrm{T}$ cells in their response to chemokines; (iii) the role of redox mechanisms in MS propagation and the remission-relapse shift; and (iv) the design of new MS models that are based on redox changes which are intrinsic to the CNS. Eventually, the results of redox studies could lead to new therapies in MS, as well as learning about lifestyle adjustments that would contribute to the patients' well-being.

\section{Acknowledgment}

This study was supported by the Ministry of Education and Science of the Republic of Serbia, grant numbers OI173014 and OI173035.

\section{References}

1. Aboul-Enein F, Rauschka H, Kornek B, Stadelmann C, Stefferl A, Brück W, Lucchinetti C, Schmidbauer M, Jellinger $\mathrm{K}$, and Lassmann H. Preferential loss of myelin-associated glycoprotein reflects hypoxia-like white matter damage in 
stroke and inflammatory brain diseases. J Neuropathol Exp Neurol 62: 25-33, 2003.

2. Adhya S, Johnson G, Herbert J, Jaggi H, Babb JS, Grossman RI, and Inglese M. Pattern of hemodynamic impairment in multiple sclerosis: dynamic susceptibility contrast perfusion MR imaging at 3.0 T. Neuroimage 33: 1029-1035, 2006.

3. Adibhatla RM and Hatcher JF. Lipid oxidation and peroxidation in CNS health and disease: from molecular mechanisms to therapeutic opportunities. Antioxid Redox Signal 12: 125-169, 2010.

4. Agrawal S, Anderson P, Durbeej M, van Rooijen N, Ivars F, Opdenakker G, and Sorokin LM. Dystroglycan is selectively cleaved at the parenchymal basement membrane at sites of leukocyte extravasation in experimental autoimmune encephalomyelitis. J Exp Med 203: 1007-1019, 2006.

5. Agrawal YP. Low dose naltrexone therapy in multiple sclerosis. Med Hypotheses 64: 721-724, 2005.

6. Ai LS and Liao F. Mutating the four extracellular cysteines in the chemokine receptor CCR6 reveals their differing roles in receptor trafficking, ligand binding, and signaling. Biochemistry 41: 8332-8341, 2002.

7. Akagawa M, Ito S, Toyoda K, Ishii Y, Tatsuda E, Shibata T, Yamaguchi S, Kawai Y, Ishino K, Kishi Y, Adachi T, Tsubata T, Takasaki Y, Hattori N, Matsuda T, and Uchida K. Bispecific abs against modified protein and DNA with oxidized lipids. Proc Natl Acad Sci U S A 103: 6160-6165, 2006.

8. Akhand AA, Du J, Liu W, Hossain K, Miyata T, Nagase F, Kato M, Suzuki H, and Nakashima I. Redox-linked cell surface-oriented signaling for T-cell death. Antioxid Redox Signal 4: 445-454, 2002.

9. Aktas O, Prozorovski T, Smorodchenko A, Savaskan NE, Lauster R, Kloetzel PM, Infante-Duarte C, Brocke S, and Zipp F. Green tea epigallocatechin-3-gallate mediates $\mathrm{T}$ cellular NF-kappa B inhibition and exerts neuroprotection in autoimmune encephalomyelitis. J Immunol 173: 57945800, 2004.

10. Albrecht P, Bouchachia I, Goebels N, Henke N, Hofstetter $\mathrm{HH}$, Issberner A, Kovacs Z, Lewerenz J, Lisak D, Maher P, Mausberg AK, Quasthoff K, Zimmermann C, Hartung HP, and Methner A. Effects of dimethyl fumarate on neuroprotection and immunomodulation. J Neuroinflammation 9: 163, 2012.

11. Almolda B, González B, and Castellano B. Activated microglial cells acquire an immature dendritic cell phenotype and may terminate the immune response in an acute model of EAE. J Neuroimmunol 223: 39-54, 2010.

12. Aloisi F, Ria F, Columba-Cabezas S, Hess H, Penna G, and Adorini L. Relative efficiency of microglia, astrocytes, dendritic cells and B cells in naïve CD4 $+\mathrm{T}$ cell priming and Th1/Th2 cell restimulation. Eur J Immunol 29: 27052714, 1999.

13. Ambrosini E, Remoli ME, Giacomini E, Rosicarelli B, Serafini B, Lande R, Aloisi F, and Coccia EM. Astrocytes produce dendritic cell-attracting chemokines in vitro and in multiple sclerosis lesions. J Neuropathol Exp Neurol 64: 706-715, 2005.

14. Anderson AC, Chandwaskar R, Lee DH, Sullivan JM, Solomon A, Rodriguez-Manzanet R, Greve B, Sobel RA, and Kuchroo VK. A transgenic model of central nervous system autoimmunity mediated by CD4 + and CD8 $+\mathrm{T}$ and B cells. J Immunol 188: 2084-2092, 2012.

15. Andrades MÉ, Morina A, Spasić S, and Spasojević I. Benchto-bedside review: sepsis-from the redox point of view. Crit Care 15: 230, 2011.
16. Angelini G, Gardella S, Ardy M, Ciriolo MR, Filomeni G, Di Trapani G, Clarke F, Sitia R, and Rubartelli A. Antigenpresenting dendritic cells provide the reducing extracellular microenvironment required for $\mathrm{T}$ lymphocyte activation. Proc Natl Acad Sci U S A 99: 1491-1496, 2002.

17. Annibali V, Ristori G, Angelini DF, Serafini B, Mechelli R, Cannoni S, Romano S, Paolillo A, Abderrahim H, Diamantini A, Borsellino G, Aloisi F, Battistini L, and Salvetti M. CD161(high)CD8 + T cells bear pathogenetic potential in multiple sclerosis. Brain 134: 542-554, 2011.

18. Antel J and Arnold D. The search for the missing links in multiple sclerosis. Curr Neurol Neurosci Rep 7: 93-94, 2007.

19. Antony JM, van Marle G, Opii W, Butterfield DA, Mallet F, Yong VW, Wallace JL, Deacon RM, Warren K, and Power C. Human endogenous retrovirus glycoprotein-mediated induction of redox reactants causes oligodendrocyte death and demyelination. Nat Neurosci 7: 1088-1095, 2004.

20. Aquino DA, Capello E, Weisstein J, Sanders V, Lopez C, Tourtellotte WW, Brosnan CF, Raine CS, and Norton WT. Multiple sclerosis: altered expression of $70-$ and $27-\mathrm{kDa}$ heat shock proteins in lesions and myelin. J Neuropathol Exp Neurol 56: 664-672, 1997.

21. Arosio P and Levi S. Ferritin, iron homeostasis, and oxidative damage. Free Radic Biol Med 33: 457-463, 2002.

22. Artemiadis AK and Anagnostouli MC. Apoptosis of oligodendrocytes and post-translational modifications of myelin basic protein in multiple sclerosis: possible role for the early stages of multiple sclerosis. Eur Neurol 63: 6572, 2010.

23. Ascherio A and Munger K. Epidemiology of multiple sclerosis: from risk factors to prevention. Semin Neurol 28: 17-28, 2008.

24. Aulkemeyer P, Hausner G, Brinkmeier H, Weber F, Würz A, Heidenreich F, and Rüdel R. The small sodium-channel blocking factor in the cerebrospinal fluid of multiple sclerosis patients is probably an oligopeptide. J Neurol Sci 172: 49-54, 2000.

25. Babbe H, Roers A, Waisman A, Lassmann H, Goebels N, Hohlfeld R, Friese M, Schröder R, Deckert M, Schmidt S, Ravid R, and Rajewsky K. Clonal expansions of CD8(+) T cells dominate the $\mathrm{T}$ cell infiltrate in active multiple sclerosis lesions as shown by micromanipulation and single cell polymerase chain reaction. J Exp Med 192: 393-404, 2000.

26. Back SA, Gan X, Li Y, Rosenberg PA, and Volpe JJ. Maturation-dependent vulnerability of oligodendrocytes to oxidative stress-induced death caused by glutathione depletion. J Neurosci 18: 6241-6253, 1998.

27. Bagnato F, Hametner S, Yao B, van Gelderen P, Merkle H, Cantor FK, Lassmann $\mathrm{H}$, and Duyn JH. Tracking iron in multiple sclerosis: a combined imaging and histopathological study at 7 Tesla. Brain 134: 3599-3612, 2011.

28. Bahbouhi B, Pettré S, Berthelot L, Garcia A, Elong Ngono A, Degauque N, Michel L, Wiertlewski S, Lefrère F, Meyniel C, Delcroix C, Brouard S, Laplaud DA, and Soulillou JP. T cell recognition of self-antigen presenting cells by protein transfer assay reveals a high frequency of anti-myelin T cells in multiple sclerosis. Brain 133: 16221636, 2010.

29. Baker CS, Hall RJ, Evans TJ, Pomerance A, Maclouf J, Creminon C, Yacoub MH, and Polak JM. Cyclooxygenase-2 is widely expressed in atherosclerotic lesions affecting native and transplanted human coronary arteries and colocalizes with inducible nitric oxide synthase and 
nitrotyrosine particularly in macrophages. Arterioscler Thromb Vasc Biol 19: 646-655, 1999.

30. Baker PR, Schopfer FJ, O'Donnell VB, and Freeman BA. Convergence of nitric oxide and lipid signaling: anti-inflammatory nitro-fatty acids. Free Radic Biol Med 46: 9891003, 2009

31. Balabanov R, Strand K, Goswami R, McMahon E, Begolka W, Miller SD, and Popko B. Interferon-gamma-oligodendrocyte interactions in the regulation of experimental autoimmune encephalomyelitis. J Neurosci 27: 2013-2024, 2007.

32. Barnett $\mathrm{MH}$ and Prineas JW. Relapsing and remitting multiple sclerosis: Pathology of the newly forming lesion. Ann Neurol 55: 458-468, 2004.

33. Barnett MH, Henderson AP, and Prineas JW. The macrophage in MS: just a scavenger after all? Pathology and pathogenesis of the acute MS lesion. Mult Scler 12: 121-132, 2006.

34. Bartholomäus I, Kawakami N, Odoardi F, Schläger C, Miljković D, Ellwart JW, Klinkert WE, Flügel-Koch C, Issekutz TB, Wekerle $\mathrm{H}$, and Flügel $\mathrm{A}$. Effector T cell interactions with meningeal vascular structures in nascent autoimmune CNS lesions. Nature 462: 94-98, 2009.

35. Bartnik BL, Juurlink BH, and Devon RM. Macrophages: their myelinotrophic or neurotoxic actions depend upon tissue oxidative stress. Mult Scler 6: 37-42, 2000.

36. Baxter AG. The origin and application of experimental autoimmune encephalomyelitis. Nat Rev Immunol 7: 904912, 2007.

37. Beauvillain C, Donnou S, Jarry U, Scotet M, Gascan H, Delneste Y, Guermonprez P, Jeannin P, and Couez D. Neonatal and adult microglia cross-present exogenous antigens. Glia 2008 56: 69-77, 2008.

38. Becher B, Bechmann I, and Greter M. Antigen presentation in autoimmunity and CNS inflammation: how T lymphocytes recognize the brain. J Mol Med 84: 532-543, 2006.

39. Becher B, Prat A, and Antel JP. Brain-immune connection: immuno-regulatory properties of CNS-resident cells. Glia 29: 293-304, 2000.

40. Bechmann I, Galea I, and Perry VH. What is the bloodbrain barrier (not)? Trends Immunol 28: 5-11, 2007.

41. Bedard K and Krause KH. The NOX family of ROS-generating NADPH oxidases: physiology and pathophysiology. Physiol Rev 87: 245-313, 2007.

42. Beebe-Dimmer JL, Pfeifer JR, Engle JS, and Schottenfeld D. The epidemiology of chronic venous insufficiency and varicose veins. Ann Epidemiol 15: 175-184, 2005.

43. Belkhiri A, Richards C, Whaley M, McQueen SA, and Orr FW. Increased expression of activated matrix metalloproteinase- 2 by human endothelial cells after sublethal $\mathrm{H}_{2} \mathrm{O}_{2}$ exposure. Lab Invest 77: 533-539, 1997.

44. Berthelot L, Laplaud DA, Pettré S, Ballet C, Michel L, Hillion $S$, Braudeau $C$, Connan $F$, Lefrère $F$, Wiertlewski $S$, Guillet JG, Brouard S, Choppin J, and Soulillou JP. Blood $\mathrm{CD} 8+\mathrm{T}$ cell responses against myelin determinants in multiple sclerosis and healthy individuals. Eur J Immunol 38: 1889-1899, 2008.

45. Billetta R, Ghahramani N, Morrow O, Prakken B, de Jong H, Meschter C, Lanza P, and Albani S. Epitope-specific immune tolerization ameliorates experimental autoimmune encephalomyelitis. Clin Immunol 145: 94-101, 2012.

46. Birnboim HC, Lemay AM, Lam DK, Goldstein R, and Webb JR. Cutting edge: MHC class II-restricted peptides containing the inflammation-associated marker 3-nitrotyr- osine evade central tolerance and elicit a robust cell-mediated immune response. J Immunol 171: 528-532, 2003.

47. Bishop A, Hobbs KG, Eguchi A, Jeffrey S, Smallwood L, Pennie C, Anderson J, and Estévez AG. Differential sensitivity of oligodendrocytes and motor neurons to reactive nitrogen species: implications for multiple sclerosis. $\mathrm{J} \mathrm{Neu}$ rochem 109: 93-104, 2009.

48. Bitsch A, Schuchardt J, Bunkowski S, Kuhlmann T, and Brück W. Acute axonal injury in multiple sclerosis. Correlation with demyelination and inflammation. Brain 123: 1174-1183, 2000.

49. Bitsch A, Wegener C, da Costa C, Bunkowski S, Reimers $\mathrm{CD}$, Prange HW, and Brück W. Lesion development in Marburg's type of acute multiple sclerosis: from inflammation to demyelination. Mult Scler 5: 138-146, 1999.

50. Bizzozero OA, DeJesus G, and Howard TA. Exposure of rat optic nerves to nitric oxide causes protein S-nitrosation and myelin decompaction. Neurochem Res 29: 1675-1685, 2004.

51. Bogie JF, Stinissen P, Hellings N, and Hendriks JJ. Myelinphagocytosing macrophages modulate autoreactive $\mathrm{T}$ cell proliferation. J Neuroinflammation 8: 85, 2011.

52. Bonetti B, Stegagno C, Cannella B, Rizzuto N, Moretto G, and Raine CS. Activation of NF-kB and c-jun transcription factors in multiple sclerosis lesions. Am J Pathol 155: 14331438, 1999.

53. Boretius S, Escher A, Dallenga T, Wrzos C, Tammer R, Brück W, Nessler S, Frahm J, and Stadelmann C. Assessment of lesion pathology in a new animal model of MS by multiparametric MRI and DTI. Neuroimage 59: 2678-2688, 2012.

54. Boullerne AI, Rodriguez JJ, Touil T, Brochet B, Schmidt S, Abrous ND, Le Moal M, Pua JR, Jensen MA, Mayo W, Arnason BG, and Petry KG. Anti-S-nitrosocysteine antibodies are a predictive marker for demyelination in experimental autoimmune encephalomyelitis: implications for multiple sclerosis. J Neurosci 22: 123-132, 2002.

55. Brambilla R, Bracchi-Ricard V, Hu WH, Frydel B, Bramwell A, Karmally S, Green EJ, and Bethea JR. Inhibition of astroglial nuclear factor kappaB reduces inflammation and improves functional recovery after spinal cord injury. J Exp Med 202: 145-156, 2005.

56. Breithaupt TB, Vazquez A, Baez I, and Eylar EH. The suppression of $\mathrm{T}$ cell function and $\mathrm{NF}$ (kappa)B expression by serine protease inhibitors is blocked by $\mathrm{N}$-acetylcysteine. Cell Immunol 173: 124-130, 1996.

57. Brennan AM, Suh SW, Won SJ, Narasimhan P, Kauppinen TM, Lee H, Edling Y, Chan PH, and Swanson RA. NADPH oxidase is the primary source of superoxide induced by NMDA receptor activation. Nat Neurosci 12: 857-863, 2009.

58. Brenner T, Brocke S, Szafer F, Sobel RA, Parkinson JF, Perez $\mathrm{DH}$, and Steinman L. Inhibition of nitric oxide synthase for treatment of experimental autoimmune encephalomyelitis. J Immunol 158: 2940-2946, 1997.

59. Brisebois M, Zehntner SP, Estrada J, Owens T, and Fournier S. A pathogenic role for CD8 $+\mathrm{T}$ cells in a spontaneous model of demyelinating disease. J Immunol 177: 2403-2411, 2006.

60. Bromley SK, Mempel TR, and Luster AD. Orchestrating the orchestrators: chemokines in control of $\mathrm{T}$ cell traffic. Nat Immunol 9: 970-980, 2008.

61. Brück W, Pförtner R, Pham T, Zhang J, Hayardeny L, Piryatinsky V, Hanisch UK, Regen T, van Rossum D, Brakelmann L, Hagemeier K, Kuhlmann T, Stadelmann C, John GR, Kramann N, and Wegner C. Reduced astrocytic NF- $\kappa$ B 
activation by laquinimod protects from cuprizone-induced demyelination. Acta Neuropathol 124: 411-424, 2012.

62. Buettner G and Jurkiewicz BA. Catalytic metals, ascorbate and free radicals: combination to avoid. Radiat Res 145: 532-541, 1996.

63. Burns J, Rosenzweig A, Zweiman B, and Lisak RP. Isolation of myelin basic protein-reactive T-cell lines from normal human blood. Cell Immunol 81: 435-440, 1983.

64. Burns SA, Lee Archer R, Chavis JA, Tull CA, Hensley LL, and Drew PD. Mitoxantrone repression of astrocyte activation: Relevance to multiple sclerosis. Brain Res 1473: 236241, 2012.

65. Cai ZY, Yan Y, and Chen R. Minocycline reduces astrocytic reactivation and neuroinflammation in the hippocampus of a vascular cognitive impairment rat model. Neurosci Bull 26: 28-36, 2010.

66. Calabrese M, De Stefano N, Atzori M, Bernardi V, Mattisi I, Barachino L, Rinaldi L, Morra A, McAuliffe MM, Perini P, Battistin L, and Gallo P. Extensive cortical inflammation is associated with epilepsy in multiple sclerosis. J Neurol 255: 581-586, 2008.

67. Calabrese V, Cornelius C, Cuzzocrea S, Iavicoli I, Rizzarelli E, and Calabrese EJ. Hormesis, cellular stress response and vitagenes as critical determinants in aging and longevity. Mol Aspects Med 32: 279-304, 2011.

68. Calabrese V, Cornelius C, Dinkova-Kostova AT, and Calabrese EJ. Vitagenes, cellular stress response, and acetylcarnitine: relevance to hormesis. Biofactors 35: 146-160, 2009.

69. Calabrese V, Cornelius C, Dinkova-Kostova AT, Calabrese EJ, and Mattson MP. Cellular stress responses, the hormesis paradigm, and vitagenes: novel targets for therapeutic intervention in neurodegenerative disorders. Antioxid Redox Signal 13: 1763-1811, 2010.

70. Calabrese V, Cornelius C, Dinkova-Kostova AT, Iavicoli I, Di Paola R, Koverech A, Cuzzocrea S, Rizzarelli E, and Calabrese EJ. Cellular stress responses, hormetic phytochemicals and vitagenes in aging and longevity. Biochim Biophys Acta 1822: 753-783, 2012.

71. Calabrese V, Cornelius C, Stella AM, and Calabrese EJ. Cellular stress responses, mitostress and carnitine insufficiencies as critical determinants in aging and neurodegenerative disorders: role of hormesis and vitagenes. Neurochem Res 35: 1880-1915, 2010.

72. Calderon TM, Eugenin EA, Lopez L, Kumar SS, Hesselgesser J, Raine CS, and Berman JW. A role for CXCL12 (SDF-1alpha) in the pathogenesis of multiple sclerosis: regulation of CXCL12 expression in astrocytes by soluble myelin basic protein. J Neuroimmunol 177: 27-39, 2006.

73. Callister ME, Pinhu L, Catley MC, Westwell AD, Newton R, Leaver SK, Quinlan GJ, Evans TW, Griffiths MJ, and Burke-Gaffney A. PMX464, a thiol-reactive quinol and putative thioredoxin inhibitor, inhibits NF-kappaB-dependent proinflammatory activation of alveolar epithelial cells. $\mathrm{Br}$ J Pharmacol 155: 661-672, 2008.

74. Candelario-Jalil E, Taheri S, Yang Y, Sood R, Grossetete M, Estrada EY, Fiebich BL, and Rosenberg GA. Cyclooxygenase inhibition limits blood-brain barrier disruption following intracerebral injection of tumor necrosis factoralpha in the rat. J Pharmacol Exp Ther 323: 488-498, 2007.

75. Cañellas AR, Gols AR, Izquierdo JR, Subirana MT, and Gairin XM. Idiopathic inflammatory-demyelinating diseases of the central nervous system. Neuroradiology 49: 393409, 2007.
76. Carlson NG and Rose JW. Antioxidants in multiple sclerosis: do they have a role in therapy? CNS Drugs 20: 433441, 2006.

77. Carrillo-de Sauvage MA, Gómez A, Ros CM, Ros-Bernal F, Martín ED, Perez-Vallés A, Gallego-Sanchez JM, Fernández-Villalba E, Barcia C Sr., Barcia C Jr,, and Herrero MT. CCL2-expressing astrocytes mediate the extravasation of $\mathrm{T}$ lymphocytes in the brain. Evidence from patients with glioma and experimental models in vivo. PLoS One 7: e30762, 2012.

78. Castro MA, Beltrán FA, Brauchi S, and Concha II. A metabolic switch in brain: glucose and lactate metabolism modulation by ascorbic acid. J Neurochem 110: 423-440, 2009.

79. Cavone L and Chiarugi A. Targeting poly(ADP-ribose) polymerase-1 as a promising approach for immunomodulation in multiple sclerosis? Trends Mol Med 18: 92100, 2012.

80. Ce P, Erkizan O, and Gedizlioglu M. Elevated HSP27 levels during attacks in patients with multiple sclerosis. Acta Neurol Scand 124: 317-320, 2011.

81. Cesarone MR, Belcaro G, Nicolaides AN, Geroulakos G, Griffin M, Incandela L, De Sanctis MT, Sabetai M, Geroulakos G, Agus G, Bavera P, Ippolito E, Leng G, Di Renzo A, Cazaubon M, Vasdekis S, Christopoulos M, and Veller M. 'Real' epidemiology of varicose veins and chronic venous disease: The San Valentino vascular screening project. Angiology 53: 119-130, 2002.

82. Chastain EM and Miller SD. Molecular mimicry as an inducing trigger for CNS autoimmune demyelinating disease. Immunol Rev 245: 227-238, 2012.

83. Chastain EM, Duncan DS, Rodgers JM, and Miller SD. The role of antigen presenting cells in multiple sclerosis. Biochim Biophys Acta 1812: 265-274, 2011.

84. Chen A, Kumar SM, Sahley CL, and Muller KJ. Nitric oxide influences injury-induced microglial migration and accumulation in the leech CNS. J Neurosci 20: 1036-1043, 2000.

85. Chiu JJ and Shien S. Effects of disturbed flow on vascular endothelium: Pathophysiological basis and clinical perspectives. Physiol Rev 91: 327-387, 2011.

86. Choi SR, Howell OW, Carassiti D, Magliozzi R, Gveric D, Muraro PA, Nicholas R, Roncaroli F, and Reynolds R. Meningeal inflammation plays a role in the pathology of primary progressive multiple sclerosis. Brain 135: 29252937, 2012.

87. Cid C and Alcazar A. Protection of oligodendrocyte precursor cells by low doses of HSP90 inhibitors in cell culture. Exp Neurol 225: 29-33, 2010.

88. Cohn M. An in depth analysis of the concept of "polyspecificity" assumed to characterize TCR/BCR recognition. Immunol Res 40: 128-147, 2008.

89. Comi G, Jeffery D, Kappos L, Montalban X, Boyko A, Rocca MA, and Filippi M. Placebo-controlled trial of oral laquinimod for multiple sclerosis. N Engl J Med 366: 1000-1009, 2012.

90. Compston A and Coles A. Multiple sclerosis. Lancet 372: 1502-1517, 2008.

91. Constantinescu CS, Farooqi N, O'Brien K, and Gran B. Experimental autoimmune encephalomyelitis (EAE) as a model for multiple sclerosis (MS). Br J Pharmacol 164: 10791106, 2011.

92. Conti A, Miscusi M, Cardali S, Germanò A, Suzuki H, Cuzzocrea $S$, and Tomasello F. Nitric oxide in the injured 
spinal cord: synthases cross-talk, oxidative stress and inflammation. Brain Res Rev 54: 205-218, 2007.

93. Copray JC, Küst BM, Mantingh-Otter I, and Boddeke HW. p75NTR independent oligodendrocyte death in cuprizoneinduced demyelination in C57BL/6 mice. Neuropathol Appl Neurobiol 31: 600-609, 2005.

94. Correale J, Peirano I, and Romano L. Benign multiple sclerosis: a new definition of this entity is needed. Mult Scler 18: 210-218, 2012.

95. Corthay A. A three-cell model for activation of naïve $\mathrm{T}$ helper cells. Scand J Immunol 64: 93-96, 2006.

96. Costantino CM, Baecher-Allan C, and Hafler DA. Multiple sclerosis and regulatory T cells. J Clin Immunol 28: 697-706, 2008.

97. Craner MJ, Newcombe J, Black JA, Hartle C, Cuzner ML, and Waxman SG. Molecular changes in neurons in multiple sclerosis: altered axonal expression of $\mathrm{Na}_{\mathrm{v}} 1.2$ and $\mathrm{Na}_{\mathrm{v}} 1.6$ sodium channels and $\mathrm{Na}^{+} / \mathrm{Ca}^{2+}$ exchanger. Proc Natl Acad Sci U S A 101: 8168-8173, 2004.

98. Cravens PD and Lipsky PE. Dendritic cells, chemokine receptors and autoimmune inflammatory diseases. Immunol Cell Biol 80: 497-505, 2002.

99. Cree BA, Kornyeyeva E, and Goodin DS. Pilot trial of lowdose naltrexone and quality of life in multiple sclerosis. Ann Neurol 68: 145-150, 2010.

100. Criqui MH, Jamosmos M, Fronek A, Denenberg JO, Langer $\mathrm{RD}$, Bergan J, and Golomb BA. Chronic venous disease in an ethnically diverse population. The San Diego population study. Am J Epidemiol 158: 448-456, 2003.

101. Cross AH, Manning PT, Keeling RM, Schmidt RE, and Misko TP. Peroxynitrite formation within the central nervous system in active multiple sclerosis. J Neuroimmunol 88 : 45-56, 1998.

102. Cuzner ML, Gveric D, Strand C, Loughlin AJ, Paemen L, Opdenakker G, and Newcombe J. The expression of tissuetype plasminogen activator, matrix metalloproteases and endogenous inhibitors in the central nervous system in multiple sclerosis: comparison of stages in lesion evolution. J Neuropathol Exp Neurol 55: 1194-1204, 1996.

103. D'Acquisto F and Crompton T. CD3 + CD4-CD8- (double negative) T cells: saviours or villains of the immune response? Biochem Pharmacol 82: 333-340, 2011.

104. D'Autréaux B and Toledano MB. ROS as signalling molecules: mechanisms that generate specificity in ROS homeostasis. Nat Rev Mol Cell Biol 8: 813-824, 2007.

105. D'Aversa TG, Eugenin EA, Lopez L, and Berman JW. Myelin basic protein induces inflammatory mediators from primary human endothelial cells and blood-brain-barrier disruption: implications for the pathogenesis of multiple sclerosis. Neuropathol Appl Neurobiol 2012 [Epub ahead of print]; DOI: 10.1111/j.1365-2990.2012.01279.x.

106. D'haeseleer M, Cambron M, Vanopdenbosch L, and De Keyser J. Vascular aspects of multiple sclerosis. Lancet Neurol 10: 657-666, 2011.

107. Dalton CM, Chard DT, Davies GR, Miszkiel KA, Altmann DR, Fernando K, Plant GT, Thompson AJ, and Miller DH. Early development of multiple sclerosis is associated with progressive grey matter atrophy in patients presenting with clinically isolated syndromes. Brain 127: 1101-1107, 2004.

108. Darvall KAL, Sam RC, Adam DJ, Silverman SH, Fegan CD, and Bradbury AW. The incidence of raised procoagulant factors and hyperhomocysteinaemia in Chinese patients with chronic venous insufficiency. Eur J Vasc Endovasc Surg 40: 260-266, 2010.
109. De Keyser J, Zeinstra E, and Frohman E. Are astrocytes central players in the pathophysiology of multiple sclerosis? Arch Neurol 60: 132-136, 2003.

110. De Oliveira-Marques V, Cyrne L, Marinho HS, and Antunes F. A quantitative study of NF-kappaB activation by $\mathrm{H}_{2} \mathrm{O}_{2}$ : relevance in inflammation and synergy with TNFalpha. J Immunol 178: 3893-3902, 2007.

111. De Stefano N, Matthews PM, Filippi M, Agosta F, De Luca M, Bartolozzi ML, Guidi L, Ghezzi A, Montanari E, Cifelli A, Federico A, and Smith SM. Evidence of early cortical atrophy in MS: relevance to white matter changes and disability. Neurology 60: 1157-1162, 2003.

112. Deeb RS, Upmacis RK, Lamon BD, Gross SS, and Hajjar DP. Maintaining equilibrium by selective targeting of cyclooxygenase pathways: promising offensives against vascular injury. Hypertension 51: 1-7, 2008.

113. del Zoppo GJ and Milner R. Integrin-matrix interactions in the cerebral microvasculature. Arterioscler Thromb Vasc Biol 26: 1966-1975, 2006.

114. Derfuss T and Meinl E. Identifying autoantigens in demyelinating diseases: valuable clues to diagnosis and treatment? Curr Opin Neurol 25: 231-238, 2012.

115. Dibaj P, Nadrigny F, Steffens H, Scheller A, Hirrlinger J, Schomburg ED, Neusch C, and Kirchhoff F. NO mediates microglial response to acute spinal cord injury under ATP control in vivo. Glia 58: 1133-1144, 2010.

116. Dieguez-Acuña FJ and Woods JS. Inhibition of NFkappaB-DNA binding by mercuric ion: utility of the non-thiol reductant, tris(2-carboxyethyl)phosphine hydrochloride (TCEP), on detection of impaired NF-kappaBDNA binding by thiol-directed agents. Toxicol In Vitro 14: 7-16, 2000.

117. Doepp F, Würfel JT, Pfueller CF, Valdueza JM, Petersen D, Paul F, and Schreiber SJ. Venous drainage in multiple sclerosis. A combined MRI and ultrasound study. Neurology 77: 1745-1751, 2011.

118. Drechsel H and Jung G. Peptide siderophores. J Pept Sci 4: 147-181, 1998.

119. Drescher KM and Sosnowska D. Being a mouse in a man's world: what TMEV has taught us about human disease. Front Biosci 13: 3775-3785, 2008.

120. Drew PD and Chavis JA. The cyclopentone prostaglandin 15-deoxy-Delta $(12,14)$ prostaglandin J2 represses nitric oxide, TNF-alpha, and IL-12 production by microglial cells. J Neuroimmunol 115: 28-35, 2001.

121. Duan Y, Gross RA, and Sheu SS. $\mathrm{Ca}^{2+}$-dependent generation of mitochondrial reactive oxygen species serves as a signal for poly(ADP-ribose) polymerase-1 activation during glutamate excitotoxicity. J Physiol 585: 741-758, 2007.

122. Duan Y, Sahley CL, and Muller KJ. ATP and NO dually control migration of microglia to nerve lesions. Dev Neurobiol 69: 60-72, 2009.

123. Dujmović I, Pekmezović T, Obrenović R, Nikolić A, Spasić M, Mostarica Stojković M, and Drulović J. Cerebrospinal fluid and serum uric acid levels in patients with multiple sclerosis. Clin Chem Lab Med 47: 848-853, 2009.

124. Duleu S, Van Der Velden C, Poulletier de Gannes F, Tranchant MC, and Geffard M. Circulating antibodies to NO- and ONOO-modified antigens in amyotrophic lateral sclerosis, Alzheimer's disease and multiple sclerosis. Immunoanalyse Biol Spécialisée 22: 273-281, 2007.

125. Dutra RC, de Souza PR, Bento AF, Marcon R, Bicca MA, Pianowski LF, and Calixto JB. Euphol prevents experimental autoimmune encephalomyelitis in mice: evidence 
for the underlying mechanisms. Biochem Pharmacol 83: 531542, 2012.

126. Dutta R, McDonough J, Yin X, Peterson J, Chang A, Torres T, Gudz T, Macklin WB, Lewis DA, Fox RJ, Rudick R, Mirnics K, and Trapp BD. Mitochondrial dysfunction as a cause of axonal degeneration in multiple sclerosis patients. Ann Neurol 59: 478-489, 2006.

127. Ebers GC. Environmental factors and multiple sclerosis. Lancet Neurol 7: 268-277, 2008.

128. Efimova O, Szankasi P, and Kelley TW. Ncf1 (p47phox) is essential for direct regulatory $\mathrm{T}$ cell mediated suppression of CD4+ effector T cells. PLoS One 6: e16013, 2011.

129. El-behi M, Rostami A, and Ciric B. Current views on the roles of Th1 and Th17 cells in experimental autoimmune encephalomyelitis. J Neuroimmune Pharmacol 5: 189-197, 2010.

130. Engelhardt B and Ransohoff RM. The ins and outs of Tlymphocyte trafficking to the CNS: anatomical sites and molecular mechanisms. Trends Immunol 26: 485-495, 2005.

131. Engelhardt B and Sorokin L. The blood-brain and the blood-cerebrospinal fluid barriers: function and dysfunction. Semin Immunopathol 31: 497-511, 2009.

132. Engelhardt B. Molecular mechanisms involved in $\mathrm{T}$ cell migration across the blood-brain barrier. J Neural Transm 113: 477-485, 2006

133. Espejo C, Penkowa M, Sáez-Torres I, Hidalgo J, García A, Montalban X, and Martínez-Cáceres EM. Interferon-gamma regulates oxidative stress during experimental autoimmune encephalomyelitis. Exp Neurol 177: 21-31, 2002.

134. Esposito G, Scuderi C, Savani C, Steardo L Jr., De Filippis $\mathrm{D}$, Cottone P, Iuvone T, Cuomo V, and Steardo L. Cannabidiol in vivo blunts beta-amyloid induced neuroinflammation by suppressing IL-1beta and iNOS expression. Br J Pharmacol 151: 1272-1279, 2007.

135. Fan B, Bordigari G, Flammer J, Killer HE, Meyer P, and Neutzner A. Meningothelial cells participate in immunological processes in the cerebrospinal fluid. J Neuroimmunol 244: 45-50, 2012.

136. Farez MF, Quintana FJ, Gandhi R, Izquierdo G, Lucas M, and Weiner HL. Toll-like receptor 2 and poly(ADP-ribose) polymerase 1 promote central nervous system neuroinflammation in progressive EAE. Nat Immunol 10: 958-964, 2009.

137. Farinotti M, Vacchi L, Simi S, Di Pietrantonj C, Brait L, and Filippini G. Dietary interventions for multiple sclerosis. Cochrane Database Syst Rev 12: CD004192, 2012.

138. Ferretti G and Bacchetti T. Peroxidation of lipoproteins in multiple sclerosis. J Neurol Sci 311: 92-97, 2011.

139. Fetler L and Amigorena S. Neuroscience. Brain under surveillance: the microglia patrol. Science 309: 392-393, 2005.

140. Fischer MT, Sharma R, Lim JL, Haider L, Frischer JM, Drexhage J, Mahad D, Bradl M, van Horssen J, and Lassmann $\mathrm{H}$. NADPH oxidase expression in active multiple sclerosis lesions in relation to oxidative tissue damage and mitochondrial injury. Brain 135: 886-899, 2012.

141. Fitzner D, Schnaars M, van Rossum D, Krishnamoorthy G, Dibaj P, Bakhti M, Regen T, Hanisch UK, and Simons M. Selective transfer of exosomes from oligodendrocytes to microglia by macropinocytosis. J Cell Sci 124: 447-458, 2011.

142. Fonseca-Kelly Z, Nassrallah M, Uribe J, Khan RS, Dine K, Dutt M, and Shindler KS. Resveratrol neuroprotection in a chronic mouse model of multiple sclerosis. Front Neurol 3: 84,2012
143. Fox RJ, Miller DH, Phillips JT, Hutchinson M, Havrdova E, Kita M, Yang M, Raghupathi K, Novas M, Sweetser MT, Viglietta V, and Dawson KT. Placebo-controlled phase 3 study of oral BG-12 or glatiramer in multiple sclerosis. $N$ Engl J Med 367: 1087-1097, 2012.

144. French HM, Reid M, Mamontov P, and Simmons RA, and Grinspan JB. Oxidative stress disrupts oligodendrocyte maturation. J Neurosci Res 87: 3076-3087, 2009.

145. Frischer JM, Bramow S, Dal-Bianco A, Lucchinetti CF, Rauschka H, Schmidbauer M, Laursen H, Sorensen PS, and Lassmann $\mathrm{H}$. The relation between inflammation and neurodegeneration in multiple sclerosis brains. Brain 132: 1175-1189, 2009.

146. Fünfschilling U, Supplie LM, Mahad D, Boretius S, Saab AS, Edgar J, Brinkmann BG, Kassmann CM, Tzvetanova ID, Möbius W, Diaz F, Meijer D, Suter U, Hamprecht B, Sereda MW, Moraes CT, Frahm J, Goebbels S, and Nave KA. Glycolytic oligodendrocytes maintain myelin and long-term axonal integrity. Nature 485: 517-521, 2012.

147. Galea I, Bechmann I, and Perry VH. What is immune privilege (not)? Trends Immunol 28: 12-18, 2007.

148. Galleano M, Aimo L, and Puntarulo S. Ascorbyl radical/ ascorbate ratio in plasma from iron overloaded rats as oxidative stress indicator. Toxicol Lett 133: 193-201, 2002.

149. Gallucci S, Lolkema M, and Matzinger P. Natural adjuvants: endogenous activators of dendritic cells. Nat Med 5: 1249-1255, 1999.

150. Gao L and Cai H. Differential roles of NOX1/NOXO1 and NOX2/p47phox complexes in endothelial responses to laminar and oscillatory shear stress. Circulation 122: A21188, 2010

151. Gard AL, Solodushko VG, Waeg G, and Majic T. 4-Hydroxynonenal, a lipid peroxidation byproduct of spinal cord injury, is cytotoxic for oligodendrocyte progenitors and inhibits their responsiveness to PDGF. Microsc Res Tech 52: 709-718, 2001.

152. Garthwaite G, Goodwin DA, Batchelor AM, Leeming K, and Garthwaite J. Nitric oxide toxicity in CNS white matter: an in vitro study using rat optic nerve. Neuroscience 109: 145-155, 2002.

153. Gay FW, Drye TJ, Dick GWA, and Esiri MM. The application of multifactorial cluster analysis in the staging of plaques in early multiple sclerosis. Identification and characterization of the primary demyelinating lesion. Brain 120: 1461-1483, 1997.

154. Ge Y, Jensen JH, Lu H, Helpern JA, Miles L, Inglese M, Babb JS, Herbert J, and Grossman RI. Quantitative assessment of iron accumulation in the deep gray matter of multiple sclerosis by magnetic field correlation imaging. Am J Neuroradiol 28: 1639-1644, 2007.

155. Ge Y, Law M, Johnson G, Herbert J, Babb JS, Mannon LJ, and Grossman RI. Dynamic susceptibility contrast perfusion MR imaging of multiple sclerosis lesions: characterizing hemodynamic impairment and inflammatory activity. Am J Neuroradiol 26: 1539-1547, 2005.

156. Ge Y, Zohrabian VM, and Grossman RI. Seven-Tesla magnetic resonance imaging. New vision of microvascular abnormalities in multiple sclerosis. Arch Neurol 65: 812-816, 2008.

157. Ge Y, Zohrabian VM, Osa EO, Xu J, Jaggi H, Herbert J, Haacke EM, and Grossman RI. Diminished visibility of cerebral venous vasculature in multiple sclerosis by susceptibility-weighted imaging at 3.0 Tesla. J Magn Reson Imaging 29: 1190-1194, 2009. 
158. Gelderman KA, Hultqvist M, Holmberg J, Olofsson P, and Holmdahl R. T cell surface redox levels determine $\mathrm{T}$ cell reactivity and arthritis susceptibility. Proc Natl Acad Sci U S A 103: 12831-12836, 2006.

159. Gerber MR and Connor JR. Do oligodendrocytes mediate iron regulation in the human brain? Ann Neurol 26: 95-98, 1989.

160. Gertsch J and Anavi-Goffer S. Methylhonokiol attenuates neuroinflammation: a role for cannabinoid receptors? J Neuroinflammation 9: 135, 2012.

161. Ghabaee M, Jabedari B, Al-E-Eshagh N, Ghaffarpour M, and Asadi F. Serum and cerebrospinal fluid antioxidant activity and lipid peroxidation in Guillain-Barre syndrome and multiple sclerosis patients. Int J Neurosci 120: 301-304, 2010.

162. Ghoreschi K, Brück J, Kellerer C, Deng C, Peng H, Rothfuss O, Hussain RZ, Gocke AR, Respa A, Glocova I, Valtcheva N, Alexander E, Feil S, Feil R, Schulze-Osthoff K, Rupec RA, Lovett-Racke AE, Dringen R, Racke MK, and Röcken M. Fumarates improve psoriasis and multiple sclerosis by inducing type II dendritic cells. J Exp Med 208: 2291-2303, 2011.

163. Giebel SJ, Menicucci G, McGuire PG, and Das A. Matrix metalloproteinases in early diabetic retinopathy and their role in alteration of the blood-retinal barrier. Lab Invest 85: 597-607, 2005.

164. Gironi M, Martinelli-Boneschi F, Sacerdote P, Solaro C, Zaffaroni M, Cavarretta R, Moiola L, Bucello S, Radaelli M, Pilato V, Rodegher M, Cursi M, Franchi S, Martinelli V, Nemni R, Comi G, and Martino G. A pilot trial of low-dose naltrexone in primary progressive multiple sclerosis. Mult Scler 14: 1076-1083, 2008.

165. Giuliani F, Goodyer CG, Antel JP, and Yong VW. Vulnerability of human neurons to T cell-mediated cytotoxicity. J Immunol 171: 368-379, 2003.

166. Glass WG and Lane TE. Functional analysis of the CC chemokine receptor 5 (CCR5) on virus-specific CD8 $+\mathrm{T}$ cells following coronavirus infection of the central nervous system. Virology 312: 407-414, 2003.

167. Glass WG and Lane TE. Functional expression of chemokine receptor CCR5 on CD4(+) T cells during virusinduced central nervous system disease. J Virol 77: 191-198, 2003.

168. Gold R, Linington C, and Lassmann H. Understanding pathogenesis and therapy of multiple sclerosis via animal models: 70 years of merits and culprits in experimental autoimmune encephalomyelitis research. Brain 129: 19531971, 2006.

169. Gomez O, Arevalo-Martin A, Garcia-Ovejero D, OrtegaGutierrez S, Cisneros JA, Almazan G, Sánchez-Rodriguez MA, Molina-Holgado F, and Molina-Holgado E. The constitutive production of the endocannabinoid 2-arachidonoylglycerol participates in oligodendrocyte differentiation. Glia 58: 1913-1927, 2010.

170. Gonsette RE, Sindic C, D'hooghe MB, De Deyn PP, Medaer R, Michotte A, Seeldrayers P, and Guillaume D. Boosting endogenous neuroprotection in multiple sclerosis: the ASsociation of Inosine and Interferon beta in relapsing- remitting Multiple Sclerosis (ASIIMS) trial. Mult Scler 16: 455-462, 2010.

171. Gonsette RE. Oxidative stress and excitotoxicity: a therapeutic issue in multiple sclerosis? Mult Scler 14: 22-34, 2008.

172. Gonzalo H, Brieva L, Tatzber F, Jové M, Cacabelos D, Cassanyé A, Lanau-Angulo L, Boada J, Serrano JC, Gon- zález C, Hernández L, Peralta S, Pamplona R, and PorteroOtin M. Lipidome analysis in multiple sclerosis reveals protein lipoxidative damage as a potential pathogenic mechanism. J Neurochem 123: 622-634, 2012.

173. Goodin DS. The causal cascade to multiple sclerosis: a model for MS pathogenesis. PLoS One 4: e4565, 2009.

174. Gourraud PA, Harbo HF, Hauser SL, and Baranzini SE. The genetics of multiple sclerosis: an up-to-date review. Immunol Rev 248: 87-103, 2012.

175. Goverman JM. Immune tolerance in multiple sclerosis. Immunol Rev 241: 228-240, 2011.

176. Grigorian A, Araujo L, Naidu NN, Place DJ, Choudhury B, and Demetriou M. N-acetylglucosamine inhibits T-helper 1 (Th1)/T-helper 17 (Th17) cell responses and treats experimental autoimmune encephalomyelitis. J Biol Chem 286: 40133-40141, 2011.

177. Guan H, Nagarkatti PS, and Nagarkatti M. CD44 reciprocally regulates the differentiation of encephalitogenic Th1/ Th17 and Th2/regulatory T cells through epigenetic modulation involving DNA methylation of cytokine gene promoters, thereby controlling the development of experimental autoimmune encephalomyelitis. J Immunol 186: 6955-6964, 2011.

178. Guy J, Ellis EA, Hope GM, and Rao NA. Antioxidant enzymes reduce loss of blood-brain barrier integrity in experimental optic neuritis. Arch Ophthalmol 107: 1359-1363, 1989.

179. Haas KL and Franz KJ. Application of metal coordination chemistry to explore and manipulate cell biology. Chem Rev 109: 4921-4960, 2009.

180. Haegele KF, Stueckle CA, Malin JP, and Sindern E. Increase of CD8 + T-effector memory cells in peripheral blood of patients with relapsing-remitting multiple sclerosis compared to healthy controls. J Neuroimmunol 183: 168-174, 2007.

181. Haider L, Fischer MT, Frischer JM, Bauer J, Höftberger R, Botond $\mathrm{G}$, Esterbauer $\mathrm{H}$, Binder $\mathrm{CJ}$, Witztum JL, and Lassmann H. Oxidative damage in multiple sclerosis lesions. Brain 134: 1914-1924, 2011.

182. Hampson AJ, Grimaldi M, Axelrod J, and Wink D. Cannabidiol and (-)Delta9-tetrahydrocannabinol are neuroprotective antioxidants. Proc Natl Acad Sci U S A 95: 8268-8273, 1998.

183. Han Y, Englert JA, Yang R, Delude RL, and Fink MP. Ethyl pyruvate inhibits nuclear factor-kappaB-dependent signaling by directly targeting p65. J Pharmacol Exp Ther 312: 1097-1105, 2005.

184. Handel AE, Giovannoni G, Ebers GC, and Ramagopalan SV. Environmental factors and their timing in adult-onset multiple sclerosis. Nat Rev Neurol 6: 156-166, 2010.

185. Handel AE, Lincoln MR, and Ramagopalan SV. Of mice and men: experimental autoimmune encephalitis and multiple sclerosis. Eur J Clin Invest 41: 1254-1258, 2011.

186. Handunnetthi L, Ramagopalan SV, and Ebers GC. Multiple sclerosis, vitamin D, and HLA-DRB1*15. Neurology 74: 1905-1910, 2010.

187. Hanisch U and Kettenman H, Microglia: active sensor and versatile effector cells in the normal and pathologic brain. Nat Neurosci 10: 1387-1394, 2007.

188. Harari O and Liao JK. Inhibition of MHC II gene transcription by nitric oxide and antioxidants. Curr Pharm Des 10: 893-898, 2004.

189. Harrison DG, Widder J, Grumbach I, Chen W, Weber M, and Searles C. Endothelial mechanotransduction, nitric 
oxide and vascular inflammation. J Internal Med 259: 351$363,2006$.

190. Harrison FE and May JM. Vitamin C function in the brain: vital role of the ascorbate transporter SVCT2. Free Radic Biol Med 46: 719-730, 2009.

191. Hauser SL and Oksenberg JR. The neurobiology of multiple sclerosis: genes, inflammation, and neurodegeneration. Neuron 52: 61-76, 2006.

192. Hawker K. Progressive multiple sclerosis: characteristics and management. Neurol Clin 29: 423-434, 2011.

193. Hayakawa K, Mishima K, Nozako M, Ogata A, Hazekawa M, Liu AX, Fujioka M, Abe K, Hasebe N, Egashira N, Iwasaki K, and Fujiwara M. Repeated treatment with cannabidiol but not Delta9-tetrahydrocannabinol has a neuroprotective effect without the development of tolerance. Neuropharmacology 52: 1079-1087, 2007.

194. Henderson AP, Barnett MH, Parratt JD, and Prineas JW. Multiple sclerosis: distribution of inflammatory cells in newly forming lesions. Ann Neurol 66: 739-753, 2009.

195. Herges K, Millward JM, Hentschel N, Infante-Duarte C, Aktas O, and Zipp F. Neuroprotective effect of combination therapy of glatiramer acetate and epigallocatechin-3-gallate in neuroinflammation. PLoS One 6: e25456, 2011.

196. Herndon RM and Kasckow J. Electron microscopic studies of cerebrospinal fluid sediment in demyelinating disease. Ann Neurol 4: 515-523, 1978.

197. Hesse A, Wagner M, Held J, Brück W, Salinas-Riester G, Hao Z, Waisman A, and Kuhlmann T. In toxic demyelination oligodendroglial cell death occurs early and is FAS independent. Neurobiol Dis 37: 362-369, 2010.

198. Heussler VT, Fernandez PC, Machado J Jr., Botteron C, and Dobbelaere DA. N-acetylcysteine blocks apoptosis induced by $\mathrm{N}$-alpha-tosyl-L-phenylalanine chloromethyl ketone in transformed T-cells. Cell Death Differ 6: 342-350, 1999.

199. Hickey WF, Hsu BL, and Kimura H. T-lymphocyte entry into the central nervous system. J Neurosci Res 28: 254260, 1991.

200. Hirrlinger J, Resch A, Gutterer JM, and Dringen R. Oligodendroglial cells in culture effectively dispose of exogenous hydrogen peroxide: comparison with cultured neurones, astroglial and microglial cells. J Neurochem 82: 635-644, 2002.

201. Hochmeister S, Zeitelhofer M, Bauer J, Nicolussi EM, Fischer MT, Heinke B, Selzer E, Lassmann H, and Bradl M. After injection into the striatum, in vitro-differentiated microglia- and bone marrow-derived dendritic cells can leave the central nervous system via the blood stream. Am J Pathol 173: 1669-1681, 2008.

202. Hoffman $\mathrm{O}$ and Weber RJ. Pathophysiology and treatment of bacterial meningitis. Ther Adv Neurol Disord 2: 1-7, 2009.

203. Holley JE, Newcombe J, Winyard PG, and Gutowski NJ. Peroxiredoxin V in multiple sclerosis lesions: predominant expression by astrocytes. Mult Scler 13: 955-961, 2007.

204. Holman DW, Klein RS, and Ransohoff RM. The bloodbrain barrier, chemokines and multiple sclerosis. Biochim Biophys Acta 1812: 220-230, 2011.

205. Holzknecht C and Röhl C. Effects of methylprednisolone and glatiramer acetate on nitric oxide formation of cytokine-stimulated cells from the rat oligodendroglial cell line OLN-93. Neuroimmunomodulation 17: 23-30, 2010.

206. Hooper DC, Scott GS, Zborek A, Mikheeva T, Kean RB, Koprowski H, and Spitsin SV. Uric acid, a peroxynitrite scavenger, inhibits CNS inflammation, blood-CNS barrier permeability changes, and tissue damage in a mouse model of multiple sclerosis. FASEB J 14: 691-698, 2000.

207. Howell OW, Reeves CA, Nicholas R, Carassiti D, Radotra B, Gentleman SM, Serafini B, Aloisi F, Roncaroli F, Magliozzi R, and Reynolds R. Meningeal inflammation is widespread and linked to cortical pathology in multiple sclerosis. Brain 134: 2755-2771, 2011.

208. Safety and efficacy study of Copaxone administered in combination with $\mathrm{N}$-acetylcysteine. http://clinicaltrials .gov/ct2/show/NCT00203099 (last accessed on 01/14/ 2013).

209. Biogen Idec's TECFIDERA ${ }^{\mathrm{TM}}$ (dimethyl fumarate) approved in US as a first-line oral treatment for multiple sclerosis. http://www.biogenidec.com/press_release_details.aspx?ID = $5981 \&$ ReqId $=1686377$ (last accessed on 01/14/2013).

210. Efficacy of L-carnitine versus placebo in the treatment of fatigue in multiple sclerosis (FACTSEP). http://www .clinicaltrials.gov/ct2/show/NCT01149525 (last accessed on $01 / 14 / 2013$ ).

211. FDA approves new multiple sclerosis treatment, Aubagio. http://www.fda.gov/NewsEvents/Newsroom/ PressAnnouncements/ucm319277.htm (last accessed on 01/14/2013).

212. MS and cannabis. http://www.pcmd.ac.uk/news.php?id= 355 (last accessed on 01/14/2013).

213. Huppert J, Closhen D, Croxford A, White R, Kulig P, Pietrowski E, Bechmann I, Becher B, Luhmann HJ, Waisman A, and Kuhlmann CR. Cellular mechanisms of IL-17induced blood-brain barrier disruption. FASEB J 24: 10231034, 2010.

214. Iaffaldano P, Lucchese $G$, and Trojano M. Treating multiple sclerosis with natalizumab. Expert Rev Neurother 11: 16831692, 2011.

215. Ifergan I, Kebir H, Alvarez JI, Marceau G, Bernard M, Bourbonnière L, Poirier J, Duquette $\mathrm{P}$, Talbot PJ, Arbour N, and Prat A. Central nervous system recruitment of effector memory CD8 + T lymphocytes during neuroinflammation is dependent on $\alpha 4$ integrin. Brain 134: 3560-3577, 2011.

216. Ifergan I, Kébir H, Bernard M, Wosik K, Dodelet-Devillers A, Cayrol R, Arbour N, and Prat A. The blood-brain barrier induces differentiation of migrating monocytes into Th17polarizing dendritic cells. Brain 131: 785-799, 2008.

217. Ignjatović A, Stević Z, Lavrnić D, Nikolić-Kokić A, Blagojević D, Spasić M, and Spasojević I. Inappropriately chelated iron in the cerebrospinal fluid of amyotrophic lateral sclerosis patients. Amyotroph Lateral Scler 13: 357362, 2012.

218. Inglese $\mathrm{M}$, Madelin $\mathrm{G}$, Oesingmann $\mathrm{N}$, Babb JS, Wu W, Stoeckel B, Herbert J, and Johnson G. Brain tissue sodium concentration in multiple sclerosis: a sodium imaging study at 3 Tesla. Brain 133: 847-857, 2010.

219. Ireland SJ, Blazek M, Harp CT, Greenberg B, Frohman EM, Davis LS, and Monson NL. Antibody-independent B cell effector functions in relapsing remitting multiple sclerosis: clues to increased inflammatory and reduced regulatory B cell capacity. Autoimmunity 45: 400-414, 2012.

220. Jack C, Antel J, Brück W, and Kuhlmann T. Contrasting potential of nitric oxide and peroxynitrite to mediate oligodendrocyte injury in multiple sclerosis. Glia 55: 926-934, 2007.

221. Jackson SH, Devadas S, Kwon J, Pinto LA, and Williams MS. T cells express a phagocyte-type NADPH oxidase that is activated after $\mathrm{T}$ cell receptor stimulation. Nat Immunol 5: 818-827, 2004. 
222. Jacobsen M, Cepok S, Quak E, Happel M, Gaber R, Ziegler A, Schock S, Oertel WH, Sommer N, and Hemmer B. Oligoclonal expansion of memory CD8 $+\mathrm{T}$ cells in cerebrospinal fluid from multiple sclerosis patients. Brain 125: 538-550, 2002.

223. Jäger A, Dardalhon V, Sobel RA, Bettelli E, and Kuchroo VK. Th1, Th17, and Th9 effector cells induce experimental autoimmune encephalomyelitis with different pathological phenotypes. J Immunol 183: 7169-7177, 2009.

224. Jendrysik MA, Vasilevsky S, Yi L, Wood A, Zhu N, Zhao Y, Koontz SM, and Jackson SH. NADPH oxidase-2 derived ROS dictates murine DC cytokine-mediated cell fate decisions during CD4 T helper-cell commitment. PLoS One 6: e28198, 2011.

225. Ji Q and Goverman J. Experimental autoimmune encephalomyelitis mediated by CD8 + T cells. Ann N Y Acad Sci 1103: 157-166, 2007.

226. Kaneko S, Wang J, Kaneko M, Yiu G, Hurrell JM, Chitnis T, Khoury SJ, and He Z. Protecting axonal degeneration by increasing nicotinamide adenine dinucleotide levels in experimental autoimmune encephalomyelitis models. J Neurosci 26: 9794-9804, 2006.

227. Kao KK and Fink MP. The biochemical basis for the antiinflammatory and cytoprotective actions of ethyl pyruvate and related compounds. Biochem Pharmacol 80: 151-159, 2010.

228. Kapoor R, Davies M, Blaker PA, Hall SM, and Smith KJ. Blockers of sodium and calcium entry protect axons from nitric oxide-mediated degeneration. Ann Neurol 53: 174180, 2003.

229. Kappos L, Gold R, Miller DH, Macmanus DG, Havrdova E, Limmroth V, Polman CH, Schmierer K, Yousry TA, Yang M, Eraksoy M, Meluzinova E, Rektor I, Dawson KT, Sandrock AW, and O'Neill GN. Efficacy and safety of oral fumarate in patients with relapsing-remitting multiple sclerosis: a multicentre, randomised, double-blind, placebocontrolled phase IIb study. Lancet 372: 1463-1472, 2008.

230. Kaushansky N, Kerlero de Rosbo N, Zilkha-Falb R, YosefHemo R, Cohen L, and Ben-Nun A. 'Multi-epitope-targeted' immune-specific therapy for a multiple sclerosis-like disease via engineered multi-epitope protein is superior to peptides. PLoS One 6: e27860, 2011.

231. Kawakami N, Lassmann S, Li Z, Odoardi F, Ritter T, Ziemssen T, Klinkert WE, Ellwart JW, Bradl M, Krivacic K, Lassmann H, Ransohoff RM, Volk HD, Wekerle H, Linington $\mathrm{C}$, and Flügel $\mathrm{A}$. The activation status of neuroantigen-specific $T$ cells in the target organ determines the clinical outcome of autoimmune encephalomyelitis. J Exp Med 199: 185-197, 2004.

232. Keegan M, König F, McClelland R, Brück W, Morales Y, Bitsch A, Panitch H, Lassmann H, Weinshenker B, Rodriguez M, Parisi J, and Lucchinetti CF. Relation between humoral pathological changes in multiple sclerosis and response to therapeutic plasma exchange. Lancet 366: 579582, 2005.

233. Kell DB. Towards a unifying, systems biology understanding of large-scale cellular death and destruction caused by poorly liganded iron: Parkinson's, Huntington's, Alzheimer's, prions, bactericides, chemical toxicology and others as examples. Arch Toxicol 84: 825-889, 2010.

234. Kellogg DL Jr., Zhao JL, and Wu Y. Roles of nitric oxide synthase isoforms in cutaneous vasodilation induced by local warming of the skin and whole body heat stress in humans. J Appl Physiol 107: 1438-1444, 2009.
235. Kessiova M, Alexandrova A, Georgieva A, Kirkova M, and Todorov S. In vitro effects of CB1 receptor ligands on lipid peroxidation and antioxidant defense systems in the rat brain. Pharmacol Rep 58: 870-875, 2006.

236. Khalil M, Langkammer C, Ropele S, Petrovic K, WallnerBlazek M, Loitfelder M, Jehna M, Bachmaier G, Schmidt R, Enzinger C, Fuchs S, and Fazekas F. Determinants of brain iron in multiple sclerosis. A quantitative 3T MRI study. Neurology 77: 1691-1697, 2011.

237. Khorooshi R, Babcock AA, and Owens T. NF-kappaBdriven STAT2 and CCL2 expression in astrocytes in response to brain injury. J Immunol 181: 7284-7291, 2008.

238. Kim do Y, Hao J, Liu R, Turner G, Shi FD, and Rho JM. Inflammation-mediated memory dysfunction and effects of a ketogenic diet in a murine model of multiple sclerosis. PLoS One 7: e35476, 2012.

239. Kim YM, Talanian RV, and Billiar TR. Nitric oxide inhibits apoptosis by preventing increases in caspase-3-like activity via two distinct mechanisms. J Biol Chem 272: 31138-31148, 1997.

240. Kinashi T. Intracellular signalling controlling integrin activation in lymphocytes. Nat Rev Immunol 5: 546559, 2005.

241. Kipp M, Clarner T, Dang J, Copray S, and Beyer C. The cuprizone animal model: new insights into an old story. Acta Neuropathol 118: 723-736, 2009.

242. Kirk J, Plumb J, Mirakhur M, and McQuaid S. Tight junctional abnormality in multiple sclerosis white matter affects all calibres of vessel and is associated with blood-brain barrier leakage and active demyelination. J Pathol 201: 319327, 2003.

243. Kivisäkk P, Mahad DJ, Callahan MK, Sikora K, Trebst C, Tucky B, Wujek J, Ravid R, Staugaitis SM, Lassmann H, and Ransohoff RM. Expression of CCR7 in multiple sclerosis: implications for CNS immunity. Ann Neurol 55: 627-638, 2004.

244. Kivisäkk P, Mahad DJ, Callahan MK, Trebst C, Tucky B, Wei T, Wu L, Baekkevold ES, Lassmann H, Staugaitis SM, Campbell JJ, and Ransohoff RM. Human cerebrospinal fluid central memory CD4 + T cells: evidence for trafficking through choroid plexus and meninges via P-selectin. Proc Natl Acad Sci U S A 100: 8389-8394, 2003.

245. Kivisäkk P, Tucky B, Wei T, Campbell JJ, and Ransohoff RM. Human cerebrospinal fluid contains CD4+ memory T cells expressing gut- or skin-specific trafficking determinants: relevance for immunotherapy. BMC Immunol 7: 14, 2006.

246. Kloppenburg M, Brinkman BM, de Rooij-Dijk HH, Miltenburg AM, Daha MR, Breedveld FC, Dijkmans BA, and Verweij $C$. The tetracycline derivative minocycline differentially affects cytokine production by monocytes and $\mathrm{T}$ lymphocytes. Antimicrob Agents Chemother 40: 934-940, 1996.

247. Kondo N, Ishii Y, Son A, Sakakura-Nishiyama J, Kwon YW, Tanito M, Nishinaka Y, Matsuo Y, Nakayama T, Taniguchi $\mathrm{M}$, and Yodoi J. Cysteine-dependent immune regulation by TRX and MIF/GIF family proteins. Immunol Lett 92: 143-147, 2004.

248. Kouhsar SS, Karami M, Tafreshi AP, Roghani M, and Nadoushan MR. Microinjection of l-arginine into corpus callosum cause reduction in myelin concentration and neuroinflammation. Brain Res 1392: 93-100, 2011.

249. Kozela E, Lev N, Kaushansky N, Eilam R, Rimmerman N, Levy R, Ben-Nun A, Juknat A, and Vogel Z. Cannabidiol 
inhibits pathogenic T cells, decreases spinal microglial activation and ameliorates multiple sclerosis-like disease in C57BL/6 mice. Br J Pharmacol 163: 1507-1519, 2011.

250. Kraaij MD, Savage ND, van der Kooij SW, Koekkoek K, Wang J, van den Berg JM, Ottenhoff TH, Kuijpers TW, Holmdahl R, van Kooten C, and Gelderman KA. Induction of regulatory $\mathrm{T}$ cells by macrophages is dependent on production of reactive oxygen species. Proc Natl Acad Sci U S A 107: 17686-17691, 2010.

251. Krishnamoorthy G and Wekerle H. EAE: an immunologist's magic eye. Eur J Immunol 39: 2031-2035, 2009.

252. Krizanac-Bengez L, Mayberg MR, and Janigro D. The cerebral vasculature as a therapeutic target for neurological disorders and the role of shear stress in vascular homeostatis and pathophysiology. Neurol Res 26: 846-853, 2004.

253. Kroenke MA, Carlson TJ, Andjelkovic AV, and Segal BM. IL-12- and IL-23-modulated T cells induce distinct types of EAE based on histology, CNS chemokine profile, and response to cytokine inhibition. J Exp Med 205: 1535-1541, 2008.

254. Kutzelnigg A, Lucchinetti CF, Stadelmann C, Brück W, Rauschka H, Bergmann M, Schmidbauer M, Parisi JE, and Lassmann $\mathrm{H}$. Cortical demyelination and diffuse white matter injury in multiple sclerosis. Brain 128: 2705-2712, 2005.

255. Kwon J, Shatynski KE, Chen H, Morand S, de Deken X, Miot F, Leto TL, and Williams MS. The nonphagocytic NADPH oxidase Duox1 mediates a positive feedback loop during T cell receptor signaling. Sci Signal 3: ra59, 2010.

256. Laboyrie PM, Roos RA, and Verspaget HW. Copper-zinc superoxide dismutase in cerebrospinal fluid: implications for oxygen-radical stress in the central nervous system. Clin Neurol Neurosurg 94: S106-S107, 1992.

257. Lane TE and Buchmeier MJ. Murine coronavirus infection: a paradigm for virus-induced demyelinating disease. Trends Microbiol 5: 9-14, 1997.

258. Lassmann $\mathrm{H}$, Brück $W$, and Lucchinetti C. Heterogeneity of multiple sclerosis pathogenesis: implications for diagnosis and therapy. Trends Mol Med 7: 115-121, 2001.

259. Lassmann H. Hypoxia-like tissue injury as a component of multiple sclerosis lesions. J Neurol Sci 206: 187-191, 2003.

260. Lavela SL, Prohaska TR, Furner S, and Weaver FM. Chronic diseases in male veterans with multiple sclerosis. Prev Chronic Dis 9: E55, 2012.

261. Law M, Saindane AM, Ge Y, Babb JS, Johnson G, Mannon LJ, Herbert J, and Grossman RI. Microvascular abnormality in relapsing-remitting multiple sclerosis: perfusion MR imaging findings in normal-appearing white matter. Radiology 231: 645-652, 2004.

262. Lee HS, Namkoong K, Kim DH, Kim KJ, Cheong YH, Kim SS, Lee WB, and Kim KY. Hydrogen peroxide-induced alterations of tight junction proteins in bovine brain microvascular endothelial cells. Microvasc Res 68: 231-238, 2004.

263. Lee J, Ryu H, Ferrante RJ, Morris SM, and Ratan RR. Translational control of inducible nitric oxide synthase expression by arginine can explain the arginine paradox. Proc Natl Acad Sci U S A 100: 4843-4848, 2003.

264. Lee SJ, Kim CE, Yun MR, Seo KW, Park HM, Yun JW, Shin HK, Bae SS, and Kim CD. 4-Hydroxynonenal enhances MMP-9 production in murine macrophages via 5-lipoxygenase-mediated activation of ERK and p38 MAPK. Toxicol Appl Pharmacol 242: 191-198, 2010.

265. Lee YJ, Choi DY, Choi IS, Kim KH, Kim YH, Kim HM, Lee K, Cho WG, Jung JK, Han SB, Han JY, Nam SY, Yun YW,
Jeong JH, Oh KW, and Hong JT. Inhibitory effect of 4-Omethylhonokiol on lipopolysaccharide-induced neuroinflammation, amyloidogenesis and memory impairment via inhibition of nuclear factor-kappaB in vitro and in vivo models. J Neuroinflammation 9: 35, 2012.

266. Lehoux S. Redox signalling in vascular responses to shear and stretch. Cardiovasc Res 71: 269-279, 2006.

267. LeVine SM. Iron deposits in multiple sclerosis and Alzheimer's disease brains. Brain Res 760: 298-303, 1997.

268. Lin SX, Lisi L, Dello Russo C, Polak PE, Sharp A, Weinberg G, Kalinin S, and Feinstein DL. The anti-inflammatory effects of dimethyl fumarate in astrocytes involve glutathione and haem oxygenase-1. ASN Neuro 3: e00055, 2011.

269. Lin YL and Lin JK. Epigallocatechin-3-gallate blocks the induction of nitric oxide synthase by down-regulating lipopolysaccharide-induced activity of transcription factor nuclear factor-kappaB. Mol Pharmacol 52: 465-472, 1997.

270. Link H. The cytokine storm in multiple sclerosis. Mult Scler 4: 12-15, 1998.

271. Linker RA, Lee DH, Ryan S, van Dam AM, Conrad R, Bista P, Zeng W, Hronowsky X, Buko A, Chollate S, Ellrichmann G, Brück W, Dawson K, Goelz S, Wiese S, Scannevin RH, Lukashev M, and Gold R. Fumaric acid esters exert neuroprotective effects in neuroinflammation via activation of the Nrf2 antioxidant pathway. Brain 134: 678-692, 2011.

272. Lipton HL. Human Vilyuisk encephalitis. Rev Med Virol 18: 347-352, 2008.

273. Lipton HL. Theiler's virus infection in mice: an unusual biphasic disease process leading to demyelination. Infect Immun 11: 1147-1155, 1975.

274. Liu D, Wang Z, Liu S, Wang F, Zhao S, and Hao A. Antiinflammatory effects of fluoxetine in lipopolysaccharide(LPS)-stimulated microglial cells. Neuropharmacology 61: 592-599, 2011.

275. Liu JSH, Zhao ML, Brosnan CF, and Lee SC. Expression of inducible nitric oxide synthase and nitrotyrosine in multiple sclerosis lesions. Am J Pathol 158: 2057-2066, 2001.

276. Liu L, Belkadi A, Darnall L, Hu T, Drescher C, Cotleur AC, Padovani-Claudio D, He T, Choi K, Lane TE, Miller RH, and Ransohoff RM. CXCR2-positive neutrophils are essential for cuprizone-induced demyelination: relevance to multiple sclerosis. Nat Neurosci 13: 319-326, 2010.

277. Liu Y, Bubolz AH, Mendoza S, Zhang DX, and Gutterman DD. $\mathrm{H}_{2} \mathrm{O}_{2}$ is the transferrable factor mediating flow-induced dilation in human coronary arterioles. Circ Res 108: 566-573, 2011.

278. Ljubisavljević S, Stojanović I, Pavlović D, Sokolović D, and Stevanović I. Aminoguanidine and N-acetyl-cysteine supress oxidative and nitrosative stress in EAE rat brains. Redox Rep 16: 166-172, 2011.

279. Locatelli G, Wörtge S, Buch T, Ingold B, Frommer F, Sobottka B, Krüger M, Karram K, Bühlmann C, Bechmann I, Heppner FL, Waisman A, and Becher B. Primary oligodendrocyte death does not elicit anti-CNS immunity. Nat Neurosci 15: 543-550, 2012.

280. Loebermann M, Winkelmann A, Hartung HP, Hengel H, Reisinger EC, and Zettl UK. Vaccination against infection in patients with multiple sclerosis. Nat Rev Neurol 8: 143-151, 2012.

281. Lopez-Diego RS and Weiner HL. Novel therapeutic strategies for multiple sclerosis-a multifaceted adversary. Nat Rev Drug Discov 7: 909-925, 2008.

282. Lovell MA, Xiong S, Xie C, Davies P, and Markesbery WR. Induction of hyperphosphorylated tau in primary rat 
cortical neuron cultures mediated by oxidative stress and glycogen synthase kinase-3. J Alzheimers Dis 6: 659-671, 2004.

283. Lucchinetti C, Brück W, Parisi J, Scheithauer B, Rodriguez $\mathrm{M}$, and Lassmann H. Heterogeneity of multiple sclerosis lesions: implications for the pathogenesis of demyelination. Ann Neurol 47: 707-717, 2000.

284. Lucchinetti CF, Popescu BF, Bunyan RF, Moll NM, Roemer SF, Lassmann H, Brück W, Parisi JE, Scheithauer BW, Giannini C, Weigand SD, Mandrekar J, and Ransohoff RM. Inflammatory cortical demyelination in early multiple sclerosis. N Engl J Med 365: 2188-2197, 2011.

285. Maalouf M, Sullivan PG, Davis L, Kim DY, and Rho JM. Ketones inhibit mitochondrial production of reactive oxygen species production following glutamate excitotoxicity by increasing NADH oxidation. Neuroscience 145: 256-264, 2007.

286. Madge LA and May MJ. Classical NF-kappaB activation negatively regulates noncanonical NF-kappaB-dependent CXCL12 expression. J Biol Chem 285: 38069-38077, 2010.

287. Magliozzi R, Howell OW, Reeves C, Roncaroli F, Nicholas R, Serafini B, Aloisi F, and Reynolds R. Gradient of neuronal loss and meningeal inflammation in multiple sclerosis. Ann Neurol 68: 477-493, 2010.

288. Mahad D, Ziabreva I, Lassmann H, and Turnbull D. Mitochondrial defects in acute multiple sclerosis lesions. Brain 131: 1722-1735, 2008.

289. Mahad DJ, Ziabreva I, Campbell G, Lax N, White K, Hanson PS, Lassmann H, and Turnbull DM. Mitochondrial changes within axons in multiple sclerosis. Brain 132: 1161$1174,2009$.

290. Malla N, Berg E, Uhlin-Hansen L, and Winberg JO. Interaction of pro-matrix metalloproteinase-9/proteoglycan heteromer with gelatin and collagen. J Biol Chem 283: 13652-13665, 2008.

291. Mansilla MJ, Montalban X, and Espejo C. Heat shock protein 70: roles in multiple sclerosis. Mol Med 18: 1018-1028, 2012.

292. Marik C, Felts PA, Bauer J, Lassmann H, and Smith KJ. Lesion genesis in a subset of patients with multiple sclerosis: a role for innate immunity? Brain 130: 2800-2815, 2007.

293. Marsicano G, Moosmann B, Hermann H, Lutz B, Behl C. Neuroprotective properties of cannabinoids against oxidative stress: role of the cannabinoid receptor CB1. J Neurochem 80: 448-456, 2002.

294. Marui N, Offermann MK, Swerlick R, Kunsch C, Rosen CA, Ahmad M, Alexander RW, and Medford RM. Vascular cell adhesion molecule-1 (VCAM-1) gene transcription and expression are regulated through an antioxidant-sensitive mechanism in human vascular endothelial cells. J Clin Invest 92: 1866-1874, 1993.

295. Matthews AE, Weiss SR, and Paterson Y. Murine hepatitis virus-a model for virus-induced CNS demyelination. J Neurovirol 8: 76-85, 2002.

296. Matysiak M, Makosa B, Walczak A, and Selmaj K. Patients with multiple sclerosis resisted to glucocorticoid therapy: abnormal expression of heat-shock protein 90 in glucocorticoid receptor complex. Mult Scler 14: 919-926, 2008.

297. Mazière C, Gomila C, and Mazière JC. Oxidized lowdensity lipoprotein increases osteopontin expression by generation of oxidative stress. Free Radic Biol Med 48: 13821387, 2010.
298. McCandless EE, Budde M, Lees JR, Dorsey D, Lyng E, and Klein RS. IL-1R signaling within the central nervous system regulates CXCL12 expression at the blood-brain barrier and disease severity during experimental autoimmune encephalomyelitis. J Immunol 183: 613-620, 2009.

299. McCandless EE, Piccio L, Woerner BM, Schmidt RE, Rubin JB, Cross AH, and Klein RS. Pathological expression of CXCL12 at the blood-brain barrier correlates with severity of multiple sclerosis. Am J Pathol 172: 799-808, 2008.

300. McCarthy DP, Richards MH, and Miller SD. Mouse models of multiple sclerosis: experimental autoimmune encephalomyelitis and Theiler's virus-induced demyelinating disease. Methods Mol Biol 900: 381-401, 2012.

301. McCracken E, Valeriani V, Simpson C, Jover T, McCulloch J, and Dewar D. The lipid peroxidation by-product 4-hydroxynonenal is toxic to axons and oligodendrocytes. J Cereb Blood Flow Metab 20: 1529-1536, 2000.

302. McFarland HF and Martin R. Multiple sclerosis: a complicated picture of autoimmunity. Nat Immunol 8: 913-919, 2007.

303. McMahon JM, McQuaid S, Reynolds R, and FitzGerald UF. Increased expression of ER stress- and hypoxia-associated molecules in grey matter lesions in multiple sclerosis. Mult Scler 18: 1437-1447, 2012.

304. Meares GP, Ma X, Qin H, and Benveniste EN. Regulation of CCL20 expression in astrocytes by IL-6 and IL-17. Glia 60: 771-781, 2012.

305. Mecha M, Torrao AS, Mestre L, Carrillo-Salinas FJ, Mechoulam R, and Guaza C. Cannabidiol protects oligodendrocyte progenitor cells from inflammation-induced apoptosis by attenuating endoplasmic reticulum stress. Cell Death Dis 3: e331, 2012.

306. Medana I, Martinic MA, Wekerle H, and Neumann $\mathrm{H}$. Transection of major histocompatibility complex class I-induced neurites by cytotoxic T lymphocytes. Am J Pathol 159: 809-815, 2001.

307. Meier P. FOXP3 + regulatory T-cells in chronic kidney disease: molecular pathways and clinical implications. Adv Exp Med Biol 665: 163-170, 2009.

308. Meier UC, Giovannoni G, Tzartos JS, and Khan G. Translational Mini-Review Series on B cell subsets in disease. B cells in multiple sclerosis: drivers of disease pathogenesis and Trojan horse for Epstein-Barr virus entry to the central nervous system? Clin Exp Immunol 167: 1-6, 2012.

309. Meiron M, Zohar Y, Anunu R, Wildbaum G, and Karin N. CXCL12 (SDF-1alpha) suppresses ongoing experimental autoimmune encephalomyelitis by selecting antigenspecific regulatory T cells. J Exp Med 205: 2643-2655, 2008.

310. Melzer N, Meuth SG, and Wiendl H. CD8 + T cells and neuronal damage: direct and collateral mechanisms of cytotoxicity and impaired electrical excitability. FASEB J 23: 3659-3673, 2009.

311. Methner A, Albrecht P, Bouchachia I, and Hartung H. Prolonged incubation with Laquinimod protects hippocampal cells from oxidative stress. Neurology 78: P02.110, 2012.

312. Metz LM, Li D, Traboulsee A, Myles ML, Duquette P, Godin J, Constantin M, and Yong VW. Glatiramer acetate in combination with minocycline in patients with relapsing-remitting multiple sclerosis: results of a Canadian, multicenter, double-blind, placebo-controlled trial. Mult Scler 15: 1183-1194, 2009.

313. Michalke B and Nischwitz V. Review on metal speciation analysis in cerebrospinal fluid-current methods and results: a review. Anal Chim Acta 682: 23-36, 2010. 
314. Miljković Dj and Mostarica Stojković M. Resistance to the induction of experimental autoimmune encephalomyelitis a tool for studying pathogenesis of the central nervous system autoimmunity. Physiol Pharmacol Acta 42: 1-18, 2006.

315. Miljković Dj, Momčilović M, Stanojević Z, Rašić D, and Mostarica-Stojković M. It is still not for the old iron: adjuvant effects of carbonyl iron in experimental autoimmune encephalomyelitis induction. J Neurochem 118: 205214, 2011.

316. Miljković Dj, Samardžić T, Mostarica Stojković M, StošićGrujičić S, Popadić D, and Trajković V. Leflunomide inhibits activation of inducible nitric oxide synthase in rat astrocytes. Brain Res 889: 331-338, 2001.

317. Miljković Dj, Stanojević Z, Momčilović M, Odoardi F, Flügel A, and Mostarica-Stojković M. CXCL12 expression within the CNS contributes to the resistance against experimental autoimmune encephalomyelitis in Albino Oxford rats. Immunobiology 216: 979-987, 2011.

318. Miljković Dj, Timotijević G, and Mostarica Stojković M. Astrocytes in the tempest of multiple sclerosis. FEBS Lett 585: 3781-3788, 2011.

319. Millonig A, Hegen H, Di Pauli F, Ehling R, Gneiss C, Hoelzl M, Künz B, Lutterotti A, Rudzki D, Berger T, Reindl M, and Deisenhammer F. Natalizumab treatment reduces endothelial activity in MS patients. J Neuroimmunol 227: 190-194, 2010.

320. Milo R and Kahana E. Multiple sclerosis: Geoepidemiology, genetics and the environment. Autoimmunity Rev 9: A387A394, 2010

321. Mitchell KM, Dotson AL, Cool KM, Chakrabarty A, Benedict $\mathrm{SH}$, and LeVine SM. Deferiprone, an orally deliverable iron chelator, ameliorates experimental autoimmune encephalomyelitis. Mult Scler 13: 1118-1126, 2007.

322. Mitrovic B, Ignarro LJ, Vinters HV, Akers MA, Schmid I, Uittenbogaart C, and Merrill JE. Nitric oxide induces necrotic but not apoptotic cell death in oligodendrocytes. Neuroscience 65: 531-539, 1995.

323. Mkhikian $\mathrm{H}$, Grigorian A, Li CF, Chen HL, Newton B, Zhou RW, Beeton C, Torossian S, Tatarian GG, Lee SU, Lau K, Walker E, Siminovitch KA, Chandy KG, Yu Z, Dennis JW, and Demetriou M. Genetics and the environment converge to dysregulate $\mathrm{N}$-glycosylation in multiple sclerosis. Nat Commun 2: 334, 2011.

324. Moharregh-Khiabani D, Blank A, Skripuletz T, Miller E, Kotsiari A, Gudi V, and Stangel M. Effects of fumaric acids on cuprizone induced central nervous system de- and remyelination in the mouse. PLoS One 5: e11769, 2010.

325. Molina-Holgado E, Vela JM, Arévalo-Martín A, Almazán G, Molina-Holgado F, Borrell J, and Guaza C. Cannabinoids promote oligodendrocyte progenitor survival: involvement of cannabinoid receptors and phosphatidylinositol-3 kinase/Akt signaling. J Neurosci 22: 9742-9753, 2002.

326. Momčilović M, Mostarica-Stojković M, and Miljković Dj. CXCL12 in control of neuroinflammation. Immunol Res 52: 53-63, 2012.

327. Moreno B, Fernandez-Diez B, Di Penta A, and Villoslada P. Preclinical studies of methylthioadenosine for the treatment of multiple sclerosis. Mult Scler 16: 1102-1108, 2010.

328. Mostert JP, Admiraal-Behloul F, Hoogduin JM, Luyendijkm J, Heersema DJ, van Buchem MA, and De Keyser J. Effects of fluoxetine on disease activity in relapsing multiple sclerosis: a double-blind, placebo-controlled, exploratory study. J Neurol Neurosurg Psychiatry 79: 1027-1031, 2008.
329. Mott RT, Ait-Ghezala G, Town T, Mori T, Vendrame M, Zeng J, Ehrhart J, Mullan M, and Tan J. Neuronal expression of CD22: novel mechanism for inhibiting microglial proinflammatory cytokine production. Glia 46: 369-379, 2004.

330. Mouzannar R, Miric SJ, Wiggins RC, and Konat GW. Hydrogen peroxide induces rapid digestion of oligodendrocyte chromatin into high molecular weight fragments. Neurochem Int 38: 9-15, 2001.

331. Munakata T, Semba U, Shibuya Y, Kuwano K, Akagi M, and Arai S. Induction of interferon-gamma production by human natural killer cells stimulated by hydrogen peroxide. J Immunol 134: 2449-2455, 1985.

332. Murakami M, Okuyama Y, Ogura H, Asano S, Arima Y, Tsuruoka M, Harada M, Kanamoto M, Sawa Y, Iwakura Y, Takatsu K, Kamimura D, and Hirano T. Local microbleeding facilitates IL-6- and IL-17-dependent arthritis in the absence of tissue antigen recognition by activated $\mathrm{T}$ cells. J Exp Med 208: 103-114, 2011.

333. Murphy MP. How mitochondria produce reactive oxygen species. Biochem J 417: 1-13, 2009.

334. Murray J, Taylor SW, Zhang B, Ghosh SS, and Capaldi RA. Oxidative damage to mitochondrial complex I due to peroxynitrite: identification of reactive tyrosines by mass spectrometry. J Biol Chem 278: 37223-37230, 2003.

335. Mycko MP, Brosnan CF, Raine CS, Fendler W, and Selmaj KW. Transcriptional profiling of microdissected areas of active multiple sclerosis lesions reveals activation of heat shock protein genes. J Neurosci Res 90: 1941-1948, 2012.

336. Nagy G, Koncz A, Fernandez D, and Perl A. Nitric oxide, mitochondrial hyperpolarization, and $\mathrm{T}$ cell activation. Free Radic Biol Med 42: 1625-1631, 2007.

337. Naidoo R and Knapp ML. Studies of lipid peroxidation products in cerebrospinal fluid and serum in multiple sclerosis and other. Clin Chem 38: 2449-2454, 1992.

338. Nakao N, Kurokawa T, Nonami T, Tumurkhuu G, Koide $\mathrm{N}$, and Yokochi T. Hydrogen peroxide induces the production of tumor necrosis factor-alpha in RAW 264.7 macrophage cells via activation of p38 and stress-activated protein kinase. Innate Immun 14: 190-196, 2008.

339. Nath N, Khan M, Paintlia MK, Singh I, Hoda MN, and Giri S. Metformin attenuated the autoimmune disease of the central nervous system in animal models of multiple sclerosis. J Immunol 182: 8005-8014, 2009.

340. Nazarewicz RR, Ziolkowski W, Vaccaro PS, and Ghafourifar P. Effect of short-term ketogenic diet on redox status of human blood. Rejuvenation Res 10: 435-440, 2007.

341. Newcombe J, Li H, and Cuzner ML. Low density lipoprotein uptake by macrophages in multiple sclerosis plaques: implications for pathogenesis. Neuropathol Appl Neurobiol 20: 152-162, 1994.

342. Niedbala W, Cai B, Liu H, Pitman N, Chang L, and Liew FY. Nitric oxide induces CD4 + CD25 + Foxp3 regulatory T cells from CD4 + CD25 T cells via p53, IL-2, and OX40. Proc Natl Acad Sci U S A 104: 15478-15483, 2007.

343. Nikić I, Merkler D, Sorbara C, Brinkoetter M, Kreutzfeldt M, Bareyre FM, Brück W, Bishop D, Misgeld T, and Kerschensteiner M. A reversible form of axon damage in experimental autoimmune encephalomyelitis and multiple sclerosis. Nat Med 17: 495-499, 2011.

344. Nitsch R, Pohl EE, Smorodchenko A, Infante-Duarte C, Aktas $\mathrm{O}$, and Zipp F. Direct impact of $\mathrm{T}$ cells on neurons revealed by two-photon microscopy in living brain tissue. J Neurosci 24: 2458-2464, 2004. 
345. Norkute A, Hieble A, Braun A, Johann S, Clarner T, Baumgartner W, Beyer C, and Kipp M. Cuprizone treatment induces demyelination and astrocytosis in the mouse hippocampus. J Neurosci Res 87: 1343-1355, 2009.

346. Okayama N, Grisham MB, Kevil CG, Eppihimer LA, Wink DA, and Alexander JS. Effect of reactive oxygen metabolites on endothelial permeability: role of nitric oxide and iron. Microcirculation 6: 107-116, 1999.

347. Oleszak EL, Zaczynska E, bhattacharjee M, Butunoi C, Legido A, and Katsetos CD. Inducible nitric oxide synthase and nitrotyrosine are found in monocytes/macrophages and/or astrocytes in acute, but not in chronic, multiple sclerosis. Clin Diagn Lab Immunol 5: 438-445, 1998.

348. Oliveira SR, Kallaur AP, Simão AN, Morimoto HK, Lopes J, Panis C, Petenucci DL, da Silva E, Cecchini R, KaimenMaciel DR, and Reiche EM. Oxidative stress in multiple sclerosis patients in clinical remission: Association with the expanded disability status scale. J Neurol Sci 321: 49-53, 2012.

349. Olson JK, Croxford JL, Calenoff MA, Dal Canto MC, and Miller SD. A virus-induced molecular mimicry model of multiple sclerosis. J Clin Invest 108: 311-318, 2001.

350. Ott M, Demisch L, Engelhardt W, and Fischer PA. Interleukin-2, soluble interleukin-2-receptor, neopterin, Ltryptophan and beta 2-microglobulin levels in CSF and serum of patients with relapsing-remitting or chronicprogressive multiple sclerosis. J Neurol 241: 108-114, 1993.

351. Pachner AR, Li L, and Narayan K. Intrathecal antibody production in an animal model of multiple sclerosis. $J$ Neuroimmunol 185: 57-63, 2007.

352. Pachner AR. Experimental models of multiple sclerosis. Curr Opin Neurol 24: 291-299, 2011.

353. Padden M, Leech S, Craig B, Kirk J, Brankin B, and McQuaid S. Differences in expression of junctional adhesion molecule-A and beta-catenin in multiple sclerosis brain tissue: increasing evidence for the role of tight junction pathology. Acta Neuropathol 113: 177-186, 2007.

354. Paintlia MK, Paintlia AS, Singh AK, and Singh I. Synergistic activity of interleukin-17 and tumor necrosis factor- $\alpha$ enhances oxidative stress-mediated oligodendrocyte apoptosis. J Neurochem 116: 508-521, 2011.

355. Paling D, Golay X, Wheeler-Kingshott C, Kapoor R, and Miller D. Energy failure in multiple sclerosis and its investigation using MR techniques. J Neurol 258: 2113-2127, 2011.

356. Pandey MK, Sung B, Kunnumakkara AB, Sethi G, Chaturvedi MM, and Aggarwal BB. Berberine modifies cysteine 179 of IkappaBalpha kinase, suppresses nuclear factorkappaB-regulated antiapoptotic gene products, and potentiates apoptosis. Cancer Res 68: 5370-5379, 2008.

357. Papadaki EZ, Mastorodemos VC, Amanakis EZ, Tsekouras KC, Papadakis AE, Tsavalas ND, Simos PG, Karantanas $\mathrm{AH}$, Plaitakis A, and Maris TG. White matter and deep gray matter hemodynamic changes in multiple sclerosis patients with clinically isolated syndrome. Magn Reson Med 68: 1932-1942, 2012.

358. Papadopoulos D, Dukes S, Patel R, Nicholas R, Vora A, and Reynolds R. Substantial archaeocortical atrophy and neuronal loss in multiple sclerosis. Brain Pathol 19: 238-253, 2009.

359. Pascarella L, Penn A, and Schmid-Schönbein GW. Venous hypertension and the inflammatory cascade: major manifestations and trigger mechanisms. Angiology 56: S3-S10, 2005.
360. Penberthy WT and Tsunoda I. The importance of NAD in multiple sclerosis. Curr Pharm Des 15: 64-99, 2009.

361. Pennisi G, Cornelius C, Cavallaro MM, Salinaro AT, Cambria MT, Pennisi M, Bella R, Milone P, Ventimiglia B, Migliore MR, Di Renzo L, De Lorenzo A, and Calabrese V. Redox regulation of cellular stress response in multiple sclerosis. Biochem Pharmacol 82: 1490-1499, 2011.

362. Petermann $\mathrm{F}$ and Korn T. Cytokines and effector $\mathrm{T}$ cell subsets causing autoimmune CNS disease. FEBS Lett 585: 3747-3757, 2011.

363. Peterson JW, Bö L, Mörk S, Chang A, and Trapp BD. Transected neurites, apoptotic neurons, and reduced inflammation in cortical multiple sclerosis lesions. Ann Neurol 50: 389-400, 2001.

364. Pilz S, Tomaschitz A, Ritz E, and Pieber TR. Vitamin D status and arterial hypertension: a systematic review. Nat Rev Cardiol 6: 621-630, 2009.

365. Pittock SJ and Rodriguez M. Benign multiple sclerosis: a distinct clinical entity with therapeutic implications. Curr Top Microbiol Immunol 318: 1-17, 2008.

366. Pittock SJ, McClelland RL, Achenbach SJ, Konig F, Bitsch A, Brück W, Lassmann H, Parisi JE, Scheithauer BW, Rodriguez M, Weinshenker BG, and Lucchinetti CF. Clinical course, pathological correlations, and outcome of biopsy proved inflammatory demyelinating disease. I Neurol Neurosurg Psychiatry 76: 1693-1697, 2005.

367. Pomeroy IM, Jordan EK, Frank JA, Matthews PM, and Esiri MM. Diffuse cortical atrophy in a marmoset model of multiple sclerosis. Neurosci Lett 437: 121-124, 2008.

368. Ponomarev ED, Shriver LP, Maresz K, and Dittel BN. Microglial cell activation and proliferation precedes the onset of CNS autoimmunity. J Neurosci Res 81: 374-389, 2005.

369. Popescu BF and Lucchinetti CF. Meningeal and cortical grey matter pathology in multiple sclerosis. BMC Neurol 12: 11, 2012.

370. Popescu BF, Bunyan RF, Parisi JE, Ransohoff RM, and Lucchinetti CF. A case of multiple sclerosis presenting with inflammatory cortical demyelination. Neurology 76: 17051710, 2011.

371. Raasch J, Zeller N, van Loo G, Merkler D, Mildner A, Erny D, Knobeloch KP, Bethea JR, Waisman A, Knust M, Del Turco D, Deller T, Blank T, Priller J, Brück W, Pasparakis $\mathrm{M}$, and Prinz M. IkB kinase 2 determines oligodendrocyte loss by non-cell-autonomous activation of NF-kB in the central nervous system. Brain 134: 1184-1198, 2011.

372. Ramagopalan SV, Dobson R, Meier UC, and Giovannoni G. Multiple sclerosis: risk factors, prodromes, and potential causal pathways. Lancet Neurol 9: 727-739, 2010.

373. Ramagopalan SV, Maugeri NJ, Handunnetthi L, Lincoln MR, Orton SM, Dyment DA, Deluca GC, Herrera BM, Chao MJ, Sadovnick AD, Ebers GC, and Knight JC. Expression of the multiple sclerosis-associated MHC class II Allele HLADRB1*1501 is regulated by vitamin D. PLoS Genet 5: e1000369, 2009.

374. Rana S and Baranski TJ. Third extracellular loop (EC3)-N terminus interaction is important for seven-transmembrane domain receptor function: implications for an activation microswitch region. J Biol Chem 285: 31472-31483, 2010.

375. Ransohoff RM and Engelhardt B. The anatomical and cellular basis of immune surveillance in the central nervous system. Nat Rev Immunol 12: 623-635, 2012.

376. Rao RK, Basuroy S, Rao VU, Karnaky Jr KJ, and Gupta A. Tyrosine phosphorylation and dissociation of occludin-ZO-1 
and E-cadherin-beta-catenin complexes from the cytoskeleton by oxidative stress. Biochem J 368: 471-481, 2002.

377. Reboldi A, Coisne C, Baumjohann D, Benvenuto F, Bottinelli D, Lira S, Uccelli A, Lanzavecchia A, Engelhardt B, and Sallusto F. C-C chemokine receptor 6-regulated entry of TH-17 cells into the CNS through the choroid plexus is required for the initiation of EAE. Nat Immunol 10: 514-523, 2009.

378. Redford EJ, Kapoor R, and Smith KJ. Nitric oxide donors reversibly block axonal conduction: demyelinated axons are especially susceptible. Brain 120: 2149-2157, 1997.

379. Rees MD, Whitelock JM, Malle E, Chuang CY, Iozzo RV, Nilasaroya A, and Davies MJ. Myeloperoxidase-derived oxidants selectively disrupt the protein core of the heparan sulfate proteoglycan perlecan. Matrix Biol 29: 63-73, 2010.

380. Regenold WT, Phatak P, Makley MJ, Stone RD, and Kling MA. Cerebrospinal fluid evidence of increased extramitochondrial glucose metabolism implicates mitochondrial dysfunction in multiple sclerosis disease progression. J Neurol Sci 275: 106-112, 2008.

381. Rodriguez M. (Ed) Advances in Multiple Sclerosis and Experimental Demyelinating Diseases. Berlin: Springer-Verlag, 2008.

382. Rognmo O, Bjørnstad TH, Kahrs C, Tjønna AE, Bye A, Haram PM, Stølen T, Slørdahl SA, and Wisløff U. Endothelial function in highly endurance-trained men: effects of acute exercise. J Strength Cond Res 22: 535-542, 2008.

383. Ropele S, de Graaf W, Khalil M, Wattjes MP, Langkammer C, Rocca MA, Rovira A, Palace J, Barkhof F, Filippi M, and Fazekas F. MRI assessment of iron deposition in multiple sclerosis. J Magn Reson Imaging 34: 13-21, 2011.

384. Rose JW, Hill KE, Watt HE, and Carlson NG. Inflammatory cell expression of cyclooxygenase- 2 in the multiple sclerosis lesion. J Neuroimmunol 149: 40-49, 2004.

385. Rothbard JB, Zhao X, Sharpe O, Strohman MJ, Kurnellas M, Mellins ED, Robinson WH, and Steinman L. Chaperone activity of $\alpha \mathrm{B}$-crystallin is responsible for its incorrect assignment as an autoantigen in multiple sclerosis. J Immunol 186: 4263-4268, 2011.

386. Ruggieri M, Pica C, Lia A, Zimatore GB, Modesto M, Di Liddo E, Specchio LM, Livrea P, Trojano M, and Avolio C. Combination treatment of glatiramer acetate and minocycline affects phenotype expression of blood monocytederived dendritic cells in multiple sclerosis patients. J Neuroimmunol 197: 140-146, 2008.

387. Sallusto F, Geginat J, and Lanzavecchia A. Central memory and effector memory $\mathrm{T}$ cell subsets: function, generation, and maintenance. Annu Rev Immunol 22: 745-763, 2004.

388. Sánchez AJ and García-Merino A. Neuroprotective agents: cannabinoids. Clin Immunol 142: 57-67, 2012.

389. Sauter B, Albert ML, Francisco L, Larsson M, Somersan S, and Bhardwaj N. Consequences of cell death: exposure to necrotic tumor cells, but not primary tissue cells or apoptotic cells, induces the maturation of immunostimulatory dendritic cells. J Exp Med 191: 423-434, 2000.

390. Saxena A, Martin-Blondel G, Mars LT, and Liblau RS. Role of CD8 T cell subsets in the pathogenesis of multiple sclerosis. FEBS Lett 585: 3758-3763, 2011.

391. Schmidt H, Williamson D, and Ashley-Koch A. HLA-DR15 haplotype and multiple sclerosis: a HuGE review. Am J Epidemiol 165: 1097-1109, 2007.

392. Schmidt MM and Dringen R: Fumaric acid diesters deprive cultured primary astrocytes rapidly of glutathione. Neurochem Int 57: 460-467, 2010.
393. Schmidt TJ, Ak M, and Mrowietz U. Reactivity of dimethyl fumarate and methylhydrogen fumarate towards glutathione and N-acetyl-L-cysteine-preparation of S-substituted thiosuccinic acid esters. Bioorg Med Chem 15: 333-342, 2007.

394. Scott GS, Kean RB, Mikheeva T, Fabis MJ, Mabley JG, Szabo C, and Hooper DC. The therapeutic effects of PJ34 [N-(6-oxo-5,6-dihydrophen-anthridin-2-yl)-N,N-dimethylacetamide. $\mathrm{HCl}]$ a selective inhibitor of poly(ADP-ribose) polymerase, in experimental allergic encephalomyelitis are associated with immunomodulation. J Pharmacol Exp Ther 310: 1053-1061, 2004.

395. Segal AW. How neutrophils kill microbes. Annu Rev Immunol 23: 197-223, 2005.

396. Selvaraj V, Soundarapandian MM, Chechneva O, Williams AJ, Sidorov MK, Soulika AM, Pleasure DE, and Deng W. PARP-1 deficiency increases the severity of disease in a mouse model of multiple sclerosis. J Biol Chem 284: 2607026084, 2009.

397. Serafini B, Rosicarelli B, Magliozzi R, Stigliano E, Capello E, Mancardi GL, and Aloisi F. Dendritic cells in multiple sclerosis lesions: maturation stage, myelin uptake, and interaction with proliferating T cells. J Neuropathol Exp Neurol 65: 124-141, 2006.

398. Serres S, Anthony DC, Jiang Y, Campbell SJ, Broom KA, Khrapitchev A, and Sibson NR. Comparison of MRI signatures in pattern I and II multiple sclerosis models. NMR Biomed 22: 1014-1024, 2009.

399. Shaked I, Tchoresh D, Gersner R, Meiri G, Mordechai S, Xiao X, Hart RP, and Schwartz M. Protective autoimmunity: interferon-gamma enables microglia to remove glutamate without evoking inflammatory mediators. $J$ Neurochem 92: 997-1009, 2005.

400. Sharma R, Fischer MT, Bauer J, Felts PA, Smith KJ, Misu T, Fujihara K, Bradl M, and Lassmann $\mathrm{H}$. Inflammation induced by innate immunity in the central nervous system leads to primary astrocyte dysfunction followed by demyelination. Acta Neuropathol 120: 223-236, 2010.

401. Shin JH, Kim SW, Jin Y, Kim ID, and Lee JK. Ethyl pyruvate-mediated Nrf2 activation and hemeoxygenase 1 induction in astrocytes confer protective effects via autocrine and paracrine mechanisms. Neurochem Int 61: 89-99, 2012.

402. Shin T, Kim S, Moon C, Wie M, and Kim H. Aminoguanidine-induced amelioration of autoimmune encephalomyelitis is mediated by reduced expression of inducible nitric oxide synthase in the spinal cord. Immunol Invest 29: 233-241, 2000.

403. Siffrin V, Radbruch H, Glumm R, Niesner R, Paterka M, Herz J, Leuenberger T, Lehmann SM, Luenstedt S, Rinnenthal JL, Laube G, Luche H, Lehnardt S, Fehling HJ, Griesbeck $\mathrm{O}$, and Zipp F. In vivo imaging of partially reversible th17 cell-induced neuronal dysfunction in the course of encephalomyelitis. Immunity 33: 424-436, 2010.

404. Sinnecker T, Bozin I, Dörr J, Pfueller CF, Harms L, Niendorf T, Brandt AU, Paul F, and Wuerfel J. Periventricular venous density in multiple sclerosis is inversely associated with T2 lesion count: a 7 Tesla MRI study. Mult Scler 2012 [Epubahead of print]; DOI: 10.1177/1352458512451941.

405. Sklavos MM, Tse HM, and Piganelli JD. Redox modulation inhibits CD8 T cell effector function. Free Radic Biol Med 45: 1477-1486, 2008.

406. Smith KJ. Newly lesioned tissue in multiple sclerosis-a role for oxidative damage? Brain 134: 1877-1881, 2011.

407. Sobottka B, Harrer MD, Ziegler U, Fischer K, Wiendl H, Hünig T, Becher B, and Goebels N. Collateral bystander 
damage by myelin-directed CD8 $+\mathrm{T}$ cells causes axonal loss. Am J Pathol 175: 1160-1166, 2009.

408. Sørensen TL, Trebst C, Kivisäkk P, Klaege KL, Majmudar A, Ravid R, Lassmann H, Olsen DB, Strieter RM, Ransohoff RM, and Sellebjerg F. Multiple sclerosis: a study of CXCL10 and CXCR3 co-localization in the inflamed central nervous system. J Neuroimmunol 127: 59-68, 2002.

409. Spasojević I, Mojović M, Stević Z, Spasić SD, Jones DR, Morina A, and Spasić MB. Bioavailability and catalytic properties of copper and iron for Fenton chemistry in human cerebrospinal fluid. Redox Rep 15: 29-35, 2010.

410. Spasojević I, Stević Z, Nikolić-Kokić A, Jones DR, Blagojević D, and Spasić MB. Different roles of radical scavengers-ascorbate and urate in the cerebrospinal fluid of amyotrophic lateral sclerosis patients. Redox Rep 15: 81-86, 2010.

411. Stankiewicz J, Panter SS, Neema M, Arora A, Batt CE, and Bakshi R. Iron in chronic brain disorders: imaging and neurotherapeutic implications. Neurotherapeutics 4: 371-386, 2007.

412. Starckx S, Van den Steen PE, Verbeek R, van Noort JM, and Opdenakker G. A novel rationale for inhibition of gelatinase B in multiple sclerosis: MMP-9 destroys alpha Bcrystallin and generates a promiscuous $\mathrm{T}$ cell epitope. J Neuroimmunol 141: 47-57, 2003.

413. Stark K, Seubert P, Lynch G, and Baudry M. Proteolytic conversion of xanthine dehydrogenase to xanthine oxidase: evidence against a role for calcium-activated protease (calpain). Biochem Biophys Res Commun 165: 858-864, 1989.

414. Staykova MA, Berven LA, Cowden WB, Willenborg DO, and Crouch MF. Nitric oxide induces polarization of actin in encephalitogenic $\mathrm{T}$ cells and inhibits their in vitro transendothelial migration in a p70S6 kinase-independent manner. FASEB J 17: 1337-1339, 2003.

415. Stefano GB, Kim E, Liu Y, Zhu W, Casares F, Mantione K, Jones DA, and Cadet P. Nitric oxide modulates microglial activation. Med Sci Monit 10: BR17-BR22, 2004.

416. Steinman L. Shifting therapeutic attention in MS to osteopontin, type 1 and type 2 IFN. Eur J Immunol 39: 2358-2360, 2009.

417. Stys PK, Zamponi GW, van Minnen J, and Geurts JJ. Will the real multiple sclerosis please stand up? Nat Rev Neurosci 13: 507-514, 2012.

418. Sun Y, Liu J, Qian F, and Xu Q. Nitric oxide inhibits T cell adhesion and migration by down-regulation of beta1integrin expression in immunologically liver-injured mice. Int Immunopharmacol 6: 616-626, 2006.

419. Takahashi A, Hanson MG, Norell HR, Havelka AM, Kono $\mathrm{K}$, Malmberg KJ, and Kiessling RV. Preferential cell death of CD8 + effector memory (CCR7-CD45RA-) T cells by hydrogen peroxide-induced oxidative stress. J Immunol 174: 6080-6087, 2005.

420. Taub DD, Conlon K, Lloyd AR, Oppenheim JJ, and Kelvin DJ. Preferential migration of activated CD4 + and CD8 + T cells in response to MIP-1 alpha and MIP-1 beta. Science 260: 355-358, 1993.

421. Tejani AM, Wasdell M, Spiwak R, Rowell G, and Nathwani S. Carnitine for fatigue in multiple sclerosis. Cochrane Database Syst Rev 5: CD007280, 2012.

422. Thayer TC, Delano M, Liu C, Chen J, Padgett LE, Tse HM, Annamali M, Piganelli JD, Moldawer LL, and Mathews CE. Superoxide production by macrophages and $\mathrm{T}$ cells is critical for the induction of autoreactivity and type 1 diabetes. Diabetes 60: 2144-2151, 2011.
423. Thorburne SK and Juurlink BH. Low glutathione and high iron govern the susceptibility of oligodendroglial precursors to oxidative stress. J Neurochem 67: 1014-1022, 1996.

424. Todorich B, Pasquini JM, Garcia CI, Paez PM, and Connor JR. Oligodendrocytes and myelination: the role of iron. Glia 57: 467-478, 2009.

425. Torkildsen O, Brunborg LA, Myhr KM, and Bø L. The cuprizone model for demyelination. Acta Neurol Scand Suppl 188: 72-76, 2008

426. Toshniwal PK and Zarling EJ. Evidence for increased lipid peroxidation in multiple sclerosis. Neurochem Res 17: 205207, 1992.

427. Town T, Nikolic V, and Tan J. The microglial "activation" continuum: from innate to adaptive responses. J Neuroinflammation 2: 24, 2005.

428. Trapp BD and Stys PK. Virtual hypoxia and chronic necrosis of demyelinated axons in multiple sclerosis. Lancet Neurol 8: 280-291, 2009.

429. Treumer F, Zhu K, Gläser R, and Mrowietz U. Dimethylfumarate is a potent inducer of apoptosis in human T cells. J Invest Dermatol 121: 1383-1388, 2003.

430. Trifilo MJ, Bergmann CC, Kuziel WA, and Lane TE. CC chemokine ligand 3 (CCL3) regulates CD8(+)-T-cell effector function and migration following viral infection. J Virol 77: 4004-4014, 2003.

431. Tsunoda I and Fujinami RS. Inside-Out versus Outside-In models for virus induced demyelination: axonal damage triggering demyelination. Springer Semin Immunopathol 24: 105-125, 2002.

432. Turko IV and Murad F. Protein nitration in cardiovascular diseases. Pharmacol Rev 54: 619-634, 2002.

433. Tverdal A. Cohort study of ethnic group and cardiovascular and total mortality over 15 years. J Clin Epidemiol 50: 719-723, 1997.

434. Ubogu EE, Cossoy MB, and Ransohoff RM. The expression and function of chemokines involved in CNS inflammation. Trends Pharmacol Sci 27: 48-55, 2006.

435. Usatyuk PV, Parinandi NL, and Natarajan V. Redox regulation of 4-hydroxy-2-nonenal-mediated endothelial barrier dysfunction by focal adhesion, adherens, and tight junction proteins. J Biol Chem 281: 35554-35566, 2006.

436. Uzawa A, Mori M, Hayakawa S, Masuda S, Nomura F, and Kuwabara S. Expression of chemokine receptors on peripheral blood lymphocytes in multiple sclerosis and neuromyelitis optica. BMC Neurol 10: 113, 2010.

437. Valentin F, Bueb JL, Kieffer P, Tschirhart E, and Atkinson J. Oxidative stress activates MMP-2 in cultured human coronary smooth muscle cells. Fundam Clin Pharmacol 19: 661667, 2005.

438. van der Goes A, Brouwer J, Hoekstra K, Roos D, van den Berg TK, and Dijkstra CD. Reactive oxygen species are required for the phagocytosis of myelin by macrophages. J Neuroimmunol 92: 67-75, 1998.

439. van der Goes A, Wouters D, Van Der Pol SM, Huizinga R, Ronken E, Adamson P, Greenwood J, Dijkstra CD, and de Vries HE. Reactive oxygen species enhance the migration of monocytes across the blood-brain barrier in vitro. FASEB J 15: 1852-1854, 2001.

440. van der Maesen K, Hinojoza JR, and Sobel RA. Endothelial cell class II major histocompatibility complex molecule expression in stereotactic brain biopsies of patients with acute inflammatory/demyelinating conditions. J Neuropathol Exp Neurol 58: 346-358, 1999. 
441. van der Star BJ, Vogel DY, Kipp M, Puentes F, Baker D, and Amor S. In vitro and in vivo models of multiple sclerosis. CNS Neurol Disord Drug Targets 11: 570-588, 2012.

442. van Faassen E and Vanin AF. Radicals for Life: The Various Forms of Nitric Oxide. Amsterdam: Elsevier, 2007.

443. van Horssen J, Drexhage JA, Flor T, Gerritsen W, van der Valk P, and de Vries HE. Nrf2 and DJ1 are consistently upregulated in inflammatory multiple sclerosis lesions. Free Radic Biol Med 49: 1283-1289, 2010.

444. van Horssen J, Schreibelt G, Drexhage J, Hazes T, Dijkstra $C D$, van der Valk $P$, and de Vries HE. Severe oxidative damage in multiple sclerosis lesions coincides with enhanced antioxidant enzyme expression. Free Radic Biol Med 45: 1729-1737, 2008.

445. van Noort JM, Baker D, and Amor S. Mechanisms in the development of multiple sclerosis lesions: reconciling autoimmune and neurodegenerative factors. CNS Neurol Disord Drug Targets 11: 556-569, 2012.

446. van Rensburg SJ, Kotze MJ, and van Toorn R. The conundrum of iron in multiple sclerosis-time for an individualised approach. Metab Brain Dis 27: 239-253, 2012.

447. van Zwam M, Wierenga-Wolf AF, Melief MJ, Schrijver B, Laman JD, and Boven LA. Myelin ingestion by macrophages promotes their motility and capacity to recruit myeloid cells. J Neuroimmunol 225: 112-117, 2010.

448. Vanderlugt CL and Miller SD. Epitope spreading in immune-mediated diseases: implications for immunotherapy. Nat Rev Immunol 2: 85-95, 2002.

449. Varga AW, Johnson G, Babb JS, Herbert J, Grossman RI, and Inglese $\mathrm{M}$. White matter hemodynamic abnormalities precede sub-cortical gray matter changes in multiple sclerosis. J Neurol Sci 282: 28-33, 2009.

450. Venken K, Hellings N, Liblau R, and Stinissen P. Disturbed regulatory $\mathrm{T}$ cell homeostasis in multiple sclerosis. Trends Mol Med 16: 58-68, 2010.

451. Verbeek MM, Westphal JR, Ruiter DJ, and de Waal RM. T lymphocyte adhesion to human brain pericytes is mediated via very late antigen-4/vascular cell adhesion molecule-1 interactions. J Immunol 154: 5876-5884, 1995.

452. Verbeek R, van Tol EA, and van Noort JM. Oral flavonoids delay recovery from experimental autoimmune encephalomyelitis in SJL mice. Biochem Pharmacol 70: 220-228, 2005.

453. Veto S, Acs P, Bauer J, Lassmann H, Berente Z, Setalo G Jr, Borgulya G, Sumegi B, Komoly S, Gallyas F Jr., and Illes Z. Inhibiting poly(ADP-ribose) polymerase: a potential therapy against oligodendrocyte death. Brain 133: 822-834, 2010.

454. Wållberg M, Bergquist J, Achour A, Breij E, and Harris RA. Malondialdehyde modification of myelin oligodendrocyte glycoprotein leads to increased immunogenicity and encephalitogenicity. Eur J Immunol 37: 1986-1995, 2007.

455. Wang XF and Cynader MS. Pyruvate released by astrocytes protects neurons from copper-catalyzed cysteine neurotoxicity. J Neurosci 21: 3322-3331, 2001.

456. Waschbisch A, Manzel A, Linker RA, and Lee DH. Vascular pathology in multiple sclerosis: mind boosting or myth busting? Exp Transl Stroke Med 3: 7, 2011.

457. Weathington NM, van Houwelingen $\mathrm{AH}$, Noerager BD, Jackson PL, Kraneveld AD, Galin FS, Folkerts G, Nijkamp FP, and Blalock JE. A novel peptide CXCR ligand derived from extracellular matrix degradation during airway inflammation. Nat Med 12: 317-323, 2006.

458. Wegner C, Esiri MM, Chance SA, Palace J, and Matthews PM. Neocortical neuronal, synaptic, and glial loss in multiple sclerosis. Neurology 67: 960-967, 2006.
459. Wekerle $H$, Linington $C$, Lassmann $H$, and Meyermann $R$. Cellular immune reactivity within the CNS. TINS 9: 271277, 1986.

460. Wekerle H. Lessons from multiple sclerosis: models, concepts, observations. Ann Rheum Dis 67: iii56-iii60, 2008.

461. Wenning GK, Stefanova N, Jellinger KA, Poewe W, and Schlossmacher MG. Multiple system atrophy: a primary oligodendrogliopathy. Ann Neurol 64: 239-246, 2008.

462. Werts C, le Bourhis L, Liu J, Magalhaes JG, Carneiro LA, Fritz JH, Stockinger S, Balloy V, Chignard M, Decker T, Philpott DJ, Ma X, and Girardin SE. Nod1 and Nod2 induce CCL5/RANTES through the NF-kappaB pathway. Eur J Immunol 37: 2499-2508, 2007.

463. Wheeler D, Bandaru VV, Calabresi PA, Nath A, and Haughey NJ. A defect of sphingolipid metabolism modifies the properties of normal appearing white matter in multiple sclerosis. Brain 131: 3092-3102, 2008.

464. Witte ME, Bø L, Rodenburg RJ, Belien JA, Musters R, Hazes T, Wintjes LT, Smeitink JA, Geurts JJ, De Vries HE, van der Valk $\mathrm{P}$, and van Horssen J. Enhanced number and activity of mitochondria in multiple sclerosis lesions. J Pathol 219: 193-204, 2009.

465. Wolburg H, Noell S, Wolburg-Buchholz K, Mack A, and Fallier-Becker P. Agrin, aquaporin-4, and astrocyte polarity as an important feature of the blood-brain barrier. Neuroscientist 15: 180-193, 2009.

466. Won HY, Sohn JH, Min HJ, Lee K, Woo HA, Ho YS, Park JW, Rhee SG, and Hwang ES. Glutathione peroxidase 1 deficiency attenuates allergen-induced airway inflammation by suppressing Th2 and Th17 cell development. Antioxid Redox Signal 13: 575-587, 2010.

467. Wu M and Tsirka SE. Endothelial NOS-deficient mice reveal dual roles for nitric oxide during experimental autoimmune encephalomyelitis. Glia 57: 1204-1215, 2009.

468. Wuerfel E, Infante-Duarte C, Glumm R, and Wuerfel JT. Gadofluorine M-enhanced MRI shows involvement of circumventricular organs in neuroinflammation. J Neuroinflammation 7: 70, 2010.

469. Wujek JR, Bjartmar C, Richer E, Ransohoff RM, Yu M, Tuohy $\mathrm{VK}$, and Trapp BD. Axon loss in the spinal cord determines permanent neurological disability in an animal model of multiple sclerosis. J Neuropathol Exp Neurol 61: 23-32, 2002.

470. Xiang Z, Lin T, and Reeves SA. 15d-PGJ2 induces apoptosis of mouse oligodendrocyte precursor cells. J Neuroinflammation 4: 18, 2007.

471. Xie S, Li J, Wang JH, Wu Q, Yang P, Hsu HC, Smythies LE, and Mountz JD. IL-17 activates the canonical NF-kappaB signaling pathway in autoimmune B cells of BXD2 mice to upregulate the expression of regulators of G-protein signaling 16. J Immunol 184: 2289-2296, 2010.

472. Xin X, Fan B, Flammer J, Miller NR, Jaggi GP, Killer HE, Meyer P, and Neutzner A. Meningothelial cells react to elevated pressure and oxidative stress. PLoS One 6: e20142, 2011.

473. Xu J, Fan G, Chen S, Wu Y, Xu XM, and Hsu CY. Methylprednisolone inhibition of TNF-alpha expression and NF-kB activation after spinal cord injury in rats. Brain Res Mol Brain Res 59: 135-142, 1998.

474. Xu LY, Yang JS, Link H, and Xiao BG. SIN-1, a nitric oxide donor, ameliorates experimental allergic encephalomyelitis in Lewis rats in the incipient phase: the importance of the time window. J Immunol 166: 5810-5816, 2001.

475. Yamazaki T, Yang XO, Chung Y, Fukunaga A, Nurieva R, Pappu B, Martin-Orozco N, Kang HS, Ma L, Panopoulos AD, Craig S, Watowich SS, Jetten AM, Tian Q, and Dong C. 
CCR6 regulates the migration of inflammatory and regulatory T cells. J Immunol 181: 8391-8401, 2008.

476. Yan S, Chai H, Wang H, Yang H, Nan B, Yao Q, and Chen C. Effects of lysophosphatidylcholine on monolayer cell permeability of human coronary artery endothelial cells. Surgery 138: 464-473, 2005.

477. Yan Z and Banerjee R. Redox remodeling as an immunoregulatory strategy. Biochemistry 49: 1059-1066, 2010.

478. Yan Z, Garg SK, and Banerjee R. Regulatory T cells interfere with glutathione metabolism in dendritic cells and $\mathrm{T}$ cells. J Biol Chem 285: 41525-41532, 2010.

479. Yan Z, Garg SK, Kipnis J, and Banerjee R. Extracellular redox modulation by regulatory $\mathrm{T}$ cells. Nat Chem Biol 5 : 721-723, 2009.

480. Yi JS, Holbrook BC, Michalek RD, Laniewski NG, and Grayson JM. Electron transport complex I is required for CD8 + T cell function. J Immunol 177: 852-862, 2006.

481. Yoshioka Y, Yamamuro A, and Maeda S. Nitric oxide at a low concentration protects murine macrophage RAW264 cells against nitric oxide-induced death via cGMP signaling pathway. Br J Pharmacol 139: 28-34, 2003.

482. Young EA, Fowler CD, Kidd GJ, Chang A, Rudick R, Fisher $\mathrm{E}$, and Trapp BD. Imaging correlates of decreased axonal $\mathrm{Na}^{+} / \mathrm{K}^{+}$ATPase in chronic multiple sclerosis lesions. Ann Neurol 63: 428-435, 2008.

483. Yun MR, Park HM, Seo KW, Lee SJ, Im DS, and Kim CD. 5Lipoxygenase plays an essential role in 4-HNE-enhanced ROS production in murine macrophages via activation of NADPH oxidase. Free Radic Res 44: 742-750, 2010.

484. Zabad RK, Metz LM, Todoruk TR, Zhang Y, Mitchell JR, Yeung M, Patry DG, Bell RB, and Yong VW. The clinical response to minocycline in multiple sclerosis is accompanied by beneficial immune changes: a pilot study. Mult Scler 13: 517-526, 2007.

485. Zajicek J, Fox P, Sanders H, Wright D, Vickery J, Nunn A, and Thompson A. Cannabinoids for treatment of spasticity and other symptoms related to multiple sclerosis (CAMS study): multicentre randomised placebo-controlled trial. Lancet 362: 1517-1526, 2003.

486. Zajicek JP and Apostu VI. Role of cannabinoids in multiple sclerosis. CNS Drugs 25: 187-201, 2011.

487. Zamboni P, Galeotti R, Menegatti E, Malagoni AM, Tacconi G, Dall'Ara S, Bartolomei I, and Salvi F. Chronic cerebrospinal venous insufficiency in patients with multiple sclerosis. J Neurol Neurosurg Psychiatry 80: 392-399, 2009.

488. Zeis T, Probst A, Steck AJ, Stadelmann C, Brück W, and Schaeren-Wiemers N. Molecular changes in white matter adjacent to an active demyelinating lesion in early multiple sclerosis. Brain Pathol 19: 459-466, 2009.

489. Zhang F, Wang S, Gan L, Vosler PS, Gao Y, Zigmond MJ, and Chen J. Protective effects and mechanisms of sirtuins in the nervous system. Prog Neurobiol 95: 373-395, 2011.

490. Zhang SM, Hernán MA, Olek MJ, Spiegelman D, Willett WC, and Ascherio A. Intakes of carotenoids, vitamin C, and vitamin $\mathrm{E}$ and MS risk among two large cohorts of women. Neurology 57: 75-80, 2001.

491. Zhang X, Tang Y, Sujkowska D, Wang J, Ramgolam V, Sospedra M, Adams J, Martin R, Pinilla C, and MarkovicPlese S. Degenerate TCR recognition and dual DR2 restriction of autoreactive T cells: implications for the initiation of the autoimmune response in multiple sclerosis. Eur J Immunol 38: 1297-1309, 2008.

492. Zhang Z, Bryan JL, DeLassus E, Chang LW, Liao W, and Sandell LJ. CCAAT/enhancer-binding protein $\beta$ and NF- $\kappa \mathrm{B}$ mediate high level expression of chemokine genes CCL3 and CCL4 by human chondrocytes in response to IL-1 $\beta . J$ Biol Chem 285: 33092-33103, 2010.

493. Zhao W, Tilton RG, Corbett JA, McDaniel ML, Misko TP, Williamson JR, Cross AH, and Hickey WF. Experimental allergic encephalomyelitis in the rat is inhibited by aminoguanidine, an inhibitor of nitric oxide synthase. J Neuroimmunol 64: 123-133, 1996.

494. Zhi L, Ustyugova IV, Chen X, Zhang Q, and Wu MX. Enhanced Th17 differentiation and aggravated arthritis in IEX-1-deficient mice by mitochondrial reactive oxygen species-mediated signaling. J Immunol 189: 1639-1647, 2012.

Address correspondence to: Dr. Ivan Spasojević

Life Sciences Department

Institute for Multidisciplinary Research Kneza Višeslava 1 11000 Belgrade Serbia

E-mail: redoxsci@gmail.com

Date of first submission to ARS Central, November 4, 2012; date of final revised submission, February 9, 2013; date of acceptance, March 9, 2013.

$$
\begin{aligned}
& \text { Abbreviations Used } \\
& \mathrm{AEF}=\text { astrocytic end-feet } \\
& \text { AICD }=\text { activation-induced cell death } \\
& \mathrm{AOS}=\text { antioxidative system } \\
& \mathrm{APC}=\text { antigen presenting cells } \\
& \mathrm{ARE}=\text { antioxidant response element } \\
& \mathrm{Asc}=\text { ascorbate } \\
& \mathrm{BBB}=\text { blood-brain barrier } \\
& \mathrm{BCSFB}=\text { blood-CSF barrier } \\
& \mathrm{CB}=\text { cannabinoid receptors } \\
& \mathrm{CCSVI}=\text { chronic cerebrospinal venous } \\
& \text { insufficiency } \\
& \mathrm{CIS}=\text { clinically isolated syndrome } \\
& \mathrm{CLNO}_{2}=\text { nitro-cholesterol linoleate } \\
& \mathrm{CNS}=\text { central nervous system } \\
& \text { COX2 = cyclooxygenase } 2 \\
& \mathrm{CSF}=\text { cerebrospinal fluid } \\
& \mathrm{CTL}=\text { cytotoxic } \mathrm{T} \text { cells } \\
& \mathrm{CuZnSOD}=\text { copper, } \text { zinc superoxide dismutase } \\
& \text { Cys }=\text { cysteine } \\
& \mathrm{DC}=\text { dendritic cells } \\
& \text { DEPMPO = 5-(diethoxyphosphoryl })-5-\text { methyl- } \\
& \text { 1-pyrroline-N-oxide } \\
& \mathrm{DNIC}=\text { dinytrosil-iron complexes } \\
& \text { Duox } 1=\text { dual oxidase } 1 \\
& \mathrm{EAE}=\text { experimental autoimmune } \\
& \text { encephalomyelitis } \\
& \mathrm{EBL}=\text { endothelial basal lamina } \\
& \mathrm{EC}=\text { endothelial cells } \\
& \mathrm{EnC}=\text { ependimal cells } \\
& \text { eNOS }=\text { endothelial NO synthase } \\
& \mathrm{EpBL}=\text { epithelial basal lamina } \\
& \mathrm{EpC}=\text { epithelial cells of choroid plexus } \\
& \mathrm{EPR}=\text { electron paramagnetic resonance }
\end{aligned}
$$




\section{Abbreviations Used (Cont.)}

ETC $=$ electron transport chain

$\mathrm{GSH}=$ glutathione

$\mathrm{H}_{2} \mathrm{O}_{2}=$ hydrogen peroxide

$\mathrm{HNE}=4$-hydroxy-2-nonenal

HO- $1=$ heme oxygenase- 1

$\mathrm{HOCl}=$ hypochlorous acid

$\mathrm{Hsp}=$ heat shock protein

IFN $=$ interferon

$\mathrm{IL}=$ interleukin

iNOS $=$ inducible NO synthase

KEAP1 $=$ Kelch-like ECH-associated protein 1

$\mathrm{MBP}=$ myelin basic protein

$\mathrm{MDA}=$ malondialdehyde

MEC $=$ meningothelial cells

$\mathrm{Mf}=$ macrophage

$\mathrm{MHC}=$ major histocompatibility complex

$\mathrm{MMP}=$ matrix metalloproteases

$\mathrm{MnSOD}=$ manganese superoxide dismutase

MOG = myelin oligodendrocyte glycoprotein

$\mathrm{MPO}=$ myeloperoxidase

$\mathrm{MRI}=$ magnetic resonance imaging

MS $=$ multiple sclerosis

$\mathrm{NAC}=\mathrm{N}$-Acetylcysteine

$\mathrm{NAWM}=$ normally appearing white matter

$\mathrm{NF}-\kappa \mathrm{B}=$ nuclear factor-kappaB

$\mathrm{NO}=$ nitric oxide

- $\mathrm{NO}_{2}=$ nitrogen dioxide radical

$\mathrm{NOX}=\mathrm{NADPH}$ oxidase

$\mathrm{Nrf} 2=$ nuclear factor-E2-related factor

$\mathrm{O}_{2}{ }^{-}=$superoxide

$\mathrm{ODC}=$ oligodendrocytes $\cdot \mathrm{OH}=$ hydroxyl radical

$\mathrm{ONOO}^{-}=$peroxynitrite

oxHDL $=$ oxidized high-density lipoproteins

oxLDL $=$ oxidized low-density lipoproteins

$\mathrm{P}=$ parenchyma

$\mathrm{PARP}=$ poly (ADP-ribose) polymerase

$\mathrm{PBL}=$ parenchymal basal lamina

$\mathrm{PC}=$ plasma cell

PLP $=$ proteolipid protein

PPMS $=$ primary progressive MS

PRMS $=$ progressive-relapsing MS

RNS $=$ reactive nitrogen species

ROS $=$ reactive oxygen species

RRMS $=$ relapsing-remitting MS

$\mathrm{SEB}=$ Staphylococcal enterotoxin B

$\mathrm{SEE}=$ Staphylococcal enterotoxin E

$-\mathrm{SH}=$ thiol group

$\mathrm{sHsp}=$ small heat shock proteins

$-\mathrm{SOH}=$ sulfenic acid

SPMS $=$ secondary progressive MS

$\mathrm{TCR}=\mathrm{T}$ cell receptor

$\mathrm{Th}=$ helper T cells

$\mathrm{TJ}=$ tight junctions

TMEV-IDD $=$ Theiler's murine encephalitis virusinduced demyelinating disease

Treg $=$ regulatory $\mathrm{T}$ cells

TSA $=$ tissue specific antigens

$\mathrm{VL}=$ vessel lumen

VRS $=$ Virchow-Robin space

$\mathrm{V} \beta=$ variable region of $\beta$ chain of TCR

$\mathrm{XO}=$ xanthine oxidase

$\mathrm{ZO}=$ zonula occludens 